Louisiana State University

LSU Digital Commons

1967

\title{
Oscillatory Field Dependence of the Knight Shift in a Monocrystal of Tin.
}

Sharif Ahmad Khan

Louisiana State University and Agricultural \& Mechanical College

Follow this and additional works at: https://digitalcommons.Isu.edu/gradschool_disstheses

\section{Recommended Citation}

Khan, Sharif Ahmad, "Oscillatory Field Dependence of the Knight Shift in a Monocrystal of Tin." (1967). LSU Historical Dissertations and Theses. 1297.

https://digitalcommons.Isu.edu/gradschool_disstheses/1297

This Dissertation is brought to you for free and open access by the Graduate School at LSU Digital Commons. It has been accepted for inclusion in LSU Historical Dissertations and Theses by an authorized administrator of LSU Digital Commons. For more information, please contact gradetd@lsu.edu. 
This dissertation has been microtilmed exactly as received

$67-13,992$

KHAN, Sharif Ahmad, 1933-

OSCILLATORY FIELD DEPENDENCE OF THE KNIGHT SHIFT IN A MONOCRYSTAL OF TIN.

Louisiana State University and Agricultural and Mechanical College, Ph.D., 1967

Physics, solid state

University Microfilms, Inc., Ann Arbor, Michigan 


\title{
Oscillatory Field Dependence of the Knight Shift in a Monocrystal of Tin
}

\author{
A Dissertation \\ Submitted to the Graduate Faculty of the \\ Louisiana State University and \\ Agricultural and Mechanical College \\ in partial fulfillment of the \\ requirements for the degree of \\ Doctor of Philosophy \\ in
}

The Department of Physics and Astronomy

by

Sharif Ahmad Khan

M.S., Muslim University Aligarh, India, 1957

May, 1967 


\section{ACKNOWLEDGMENTS}

The author wishes to thank and express his appreciation to his research director, Professor Joseph M. Reynolds for suggesting this research and for his encouragement, advice and patient support throughout its completion.

Thanks are due and grateful acknowledgment is accorded to Dr. Roy Gordon Goodrich for his invaluable and continuous help in all phases of this work and also for providing valuable comments upon reading this manuscript.

The author wishes to express deep gratitude to Professor Claude G. Grenier for his invaluable aid in analyzing the data and for his stimulating suggestions throughout the course of this investigation.

Thanks are due to Dr. M. L. Glasser of Bat tele Memorial Institute, Columbus, Ohio, to Dr. J. J. Vuillemin of the University of Arizona, to Dr.W. G. Chambers of England (then at Rice University, Texas), and to Dr. John L. Fry of L.S.U., for many helpful discussions. The author also wishes to thank Dr. Nadim H. Zebouni and other members of the Low Temperature Laboratory for encouragement and help.

The author wishes to acknowledge the technical assistance of Mr. Leslie S. Edelen of the Physics Department, of Mr. Tom Ware Beal1, Jr. (now at Union Carbide Corporation, Indianapolis, Indiana) in the design and construction of the experimental 
apparatus, and of Mr. Bobby Kirby of the College of Physics and Chemistry in setting up the low temperature system.

The author is indebted to Mr. Hamid R. Khan for his help in the preparation and running of the experiment, and to Pat Mills for typing and proofreading this manuscript.

The author also wishes to acknowledge the Atomic Energy Commission for financial support without which this work would not have been possible. The financial assistance received from the Charles E. Coates Memorial Fund of the L.S.U. Foundation donated by George E. Coates for the preparation of this manuscript is gratefully acknowledged. 


\section{TABLE OF CONTENTS}

Page

1. INTRODUCTION

II. EXPERIMENTAL DETAILS 7

III. RESULTS 10

IV. DISCUSSION 22

A. Comparison of Periods of Oscillations with the Fermi Surface of Tin 22

B. Amplitude of the Oscillations 23

C. Effect of Magnetic Breakdown 30

V. CONCLUSION 33

APPENDIX I, ADDITIONAL EXPERIMENTAL DETAILS 34

APPENDIX II, FERMI SURFACE OF TIN 52

APPENDIX III, THEORETICAL DETAILS 57

A. General Considerations 57

B. Hamiltonian of the Electron-Nuclear System 60

C. Stephen's Theory 62

D. Glasser's Theory 77

E. Theory of Dolgopolov and Bystrik 95

$\begin{array}{lr}\text { REFERENCES } & 104\end{array}$

$\begin{array}{ll}\text { VITA } & 108\end{array}$ 


\section{LIST OF TABLES}

Page

Table

1. Values of the observed amplitude of the oscillatory Knight shift as compared to the various theoretical est imates. 


\section{LIST OF FIGURES}

Figure

Page

1. The Knight shift versus reciprocal field for four ranges of the magnetic field applied along the [001] direction.

2. Reciprocal field values of maximum Knight shift versus integers. The upper curve is for the short period oscillations $\left(3.0 \times 10^{-7} \mathrm{G}^{-1}\right)$ and the lower curve is for the long period oscillations $\left(5.9 \times 10^{-7} \mathrm{G}^{-1}\right)$.

3. Plot of the amplitude of the Knight shift oscillations versus magnetic field strength for the field along the [001] axis. The upper curve is for the short period oscillations $\left(3.0 \times 10^{-7} \mathrm{G}^{-1}\right)$, and the lower curve is for the long period oscillations $\left(5.9 \times 10^{-7} \mathrm{G}^{-1}\right)$.

4. Angular variation of the measured periods of the Knight shift oscillations for the magnetic field applied in the (100) plane. The upper curve is for the long period oscillations $\left(5.9 \times 10^{-7} \mathrm{G}^{-1}\right)$, and the lower curve is for the short period oscillations $\left(3 \times 10^{-7} \mathrm{G}^{-1}\right)$. 
5. Variation of amplitudes with orientation of magnetic field in the (100) plane. The upper curve is for the short period oscillations $\left(3.0 \times 10^{-7} \mathrm{G}^{-1}\right)$ and the lower curve is for the long period oscillations $\left(5.9 \times 10^{-7} \mathrm{G}^{-1}\right)$. The error of measurement in the upper curve is approximately the same as is indicated for the lower curve.

6. The square of the period of the long period $\left(5.9 \times 10^{-7} \mathrm{G}^{-1}\right)$ oscillations versus the square of the cosine of the angle between the magnetic field and the $[001]$ axis.

7. The average Knight shift versus reciprocal field for the field directed along the [001] axis.

8. Recorder trace of the second derivative with respect to $H$ of the magnetization as a function of the magnetic field for the field directed along the $[001]$ axis.

9. Variation of the computed amplitude of the de Haas-van Alphen susceptibility as a function of the magnetic field strength, the field being along the $[001]$ axis. The upper curve is for the long period oscillations $\left(5.9 \times 10^{-7} \mathrm{G}^{-1}\right)$ and the lower curve is for the short period oscillations $\left(3.0 \times 10^{-7} \mathrm{G}^{-1}\right)$. 
Figure

10. Energy level diagram for the spin splitting of Landau levels near the Fermi level in the presence of a strong magnetic field. Levels are shown for electrons on both the $3^{6}$, and $4 a \zeta$ orbits of the Fermi surface.

11. A circuit diagram of the power supply to the Tektronix type 160 waveform generator.

12. A circuit diagram of the Pound-Knight-Watkins marginal oscillator.

13. A circuit diagram of the Watkins calibrator.

14. A diagram of the sample holder assembly.

15. A block diagram of the experimental equipment. 44

16. A recorder trace of the derivative of the NMR signal of $\mathrm{Sn}^{119}$.

17. Sketch of the free electron model of the tin Fermi surface in the reduced zone scheme.

18. Basal cross section of the Fermi surface of tin in the extended zone scheme showing the freak orbit (i) generated as a result of magnetic breakdown connecting orbits $3 \delta_{1}$ and $4 a \zeta$

19. Integration contour of Eq. (29) of Appendix III C.

20. Fermi surface geometry for the case $a(\underline{K})>1$, (Appendix $\||| 0)$. 
ABSTRACT

A detailed experimental investigation of the magnetic field dependence of the Knight shift has been performed in a monocrystal of white tin. The measurements were made at $1.2^{\circ} \mathrm{K}$ and in magnetic fields ranging from 10.5 to $16.2 \mathrm{kG}$. The knight shift is found to oscillate at the de Has-van Alphen frequency and in the range of fields investigated exhibits two periods of $3 \times 10^{-7}$ and $5.9 \times 10^{-7} \mathrm{G}^{-1}$. The values and angular variations of these periods are in satisfactory agreement with the data on the tin Fermi surface obtained from other experiments and from a pseudopotential calculation of the band structure. The magnetic field dependence of the amplitude of the oscillations has been investigated for both periods. The amplitude of the shorter period component increases and that of the longer period component decreases with increasing magnetic field. The decrease in amplitude of the longer period oscillation is attributed to the effects of magnetic breakdown. The observed amplitudes are in satisfactory agreement with the assumption that the Knight shift oscillations are due to oscillations in the ground state wave functions of the contributing electrons rather than oscillations in the susceptibility as has been previously suggested. Measurements of the de Haas-van Alphen susceptibility in the same sample used for 
the Knight shift measurements were performed and give further support to this viewpoint since the field variation of the amplitude of the oscillations in the susceptibility is different from the corresponding field dependence of the oscillatory Knight shift. 


\section{INTRODUCTION}

In an earlier note the results of a field dependent study of the Knight shift, $\sigma$, in a monocrystal of tin were reported. It was found that above approximately $11 \mathrm{kG} \sigma$ exhibited easily observable oscillations periodic in reciprocal field. The results of an extended investigation of $\sigma$ in a monocrystal of white tin at $1.2^{\circ} \mathrm{K}$ are reported here.

The major contribution to $\sigma$ comes from the hyperfine interaction of the nuclear magnetic moment with the spins of the electrons occupying states close to the Fermi level. The difference in the observed NMR frequency, $\nu$, for a given nucleus situated in a conductor and the free atom frequency, $\nu_{0}$, divided by $\nu_{0}$ is given essentially by the magnetic shielding constant

$$
\frac{\nu-\nu_{0}}{\nu_{0}}=\sigma=-H^{-1}\left(\delta F / \delta_{\mu_{n}}\right) \mu_{n=0}
$$

where $F$ is the free energy, $H$ is the applied magnetic field and $\mu_{n}$ is the nuclear magnetic moment. In the limit of small magnetic fields and considering only spin effects this expression may be evaluated to first order in $\mu_{n}$ to yield Townes, Herring and Knight's ${ }^{2}$ expression

$$
\sigma=(8 \pi / 3) x_{p}\left\langle|\psi(0)|^{2}\right\rangle a v^{2}
$$

for the knight shift, where $\Omega$ is the atomic volume and 
where $X_{p}$ is the paramagnetic spin susceptibility of the conduction electrons, and $\left\langle|\psi(0)|^{2}\right\rangle_{\text {av }}$ is the average electron density at the nucleus of electrons at the Fermi surface. Das and Sondheimer ${ }^{3}$ have evaluated Eq. (1) including both the spin and orbital moments of the conduction electrons and obtain for free electrons

$$
\sigma=(8 \pi / 3)\left(x_{d}+x_{p}\right)
$$

where $x_{d}$ is the diamagnetic susceptibility of the conduction electrons due to their orbital motion in a magnetic field. These authors then conjectured that Eq. (3) would remain valid for the oscillatory as well as the steady part of the susceptibility and that an oscillatory knight shift due to oscillations in the density of states at the Fermi surface might be observable. Oscillations in $\sigma$ from this effect have been further commented on by Kaplan ${ }^{4}$ and Rodriquez. 5 These estimates show that the Knight shift oscillations arising from the variation of the density of electron states should be observable in fields of the order of $3 \times 10^{4}$ gauss and at temperatures of about $2^{\circ} \mathrm{K}$.

A more detailed calculation of this effect by stephen ${ }^{6}$ shows that the major contribution to the oscillatory component comes from the diamagnetic term in Eq. (3) and for free electrons the paramagnetic component has no oscillatory terms. The amplitude of the oscillatory diamagnetic term is independent of the applied field and the paramagnetic term exhibits oscillations for parabolic energy bands increasing in amplitude as $H^{1 / 2}$. 
Using a generalization of the effective mass approximation, Dogolpolov and Bystrik ${ }^{7}$ arrived at the same conclusions as Stephen concerning the field dependence of the amplitude of the oscillatory part of $\sigma$ and show that for a chemical potential of order $10^{-13}$ ergs and magnetic fields of order $10^{4}$ gauss the oscillatory component should be $\widetilde{\sigma} \sim 10^{-3} \sigma$.

In all of the above considerations the oscillatory component is assumed to arise from the susceptibility factor in Eq. (2) with the wave function factor remaining independent of the applied field. The fact that in metals the conduction electron ground state wave functions are not independent of the applied magnetic field has been the subject of much discussion in the past few years in connection with the interpretation of experiments which measure the various properties of the Fermi surface of metals and semiconductors, and especially when the metal is exhibiting magnetic breakdown. ${ }^{8}$ Recently Glasser ${ }^{9}$ has considered the first order effects of the lattice potential on the ground state wave functions (of electrons in metals) as a function of magnetic field and has shown that reasonable agreement with the Knight shift experiments can be obtained in this manner for sufficiently high magnetic fields.

Knight shift measurements on powdered samples of tin were first reported by McGarvey and Gutowsky ${ }^{10}$ and later by Bloembergen and Rowland ${ }^{11}$ and then by Karimov and Schegolev. 12 The first monocrystal experiments were performed by Jones and 
Williams ${ }^{13}$ who determined the anisotropy of both the Knight shift and the NMR line width in white tin. During the course of their experiments a preliminary search for the oscillatory component of the Knight shift was carried out but no reproducible effect could be determined over the rather limited field range that they investigated. 14 


\section{EXPERIMENTAL DETAILS}

The sample was prepared from a zone-refined bar of white tin obtained from Cominco Products Incorporated, (quoted purity: 99.9999\%). The bar contained a large crystal which was cut out, oriented and reshaped into a parallelopiped approximately $20 \mathrm{~mm} \times 17 \mathrm{~mm} \times 10 \mathrm{~mm}$. The tetragonal axis was within $1^{\circ}$ of the normal to the largest face. Laue back reflection $X$-ray photographs were used for orienting the crystal and cutting and planing was done by spark erosion. Although a light acid etch indicated that the entire piece was single crystal, $x$-ray photographs were taken at several points to assure that the crystal was not twinned. This piece was then cut into 25 wafers of about the same thickness with the $[001]$ direction contained in the plane of the wafers and perpendicular to an edge. Identifying marks were made on one of the surfaces of the parallelopiped before it was cut so that the wafers could be reassembled with the axes orientation preserved. The spark damage was removed by etching in $40 \%$ hydrochloric acid and $10 \%$ nitric acid. The final average size of the wafers was $17 \mathrm{~mm} \times 10 \mathrm{~mm} \times 0.3 \mathrm{~mm}$. These wafers were sandwiched together in proper order with a $0.013 \mathrm{~mm}$ thick mylar film placed between each of them. Small quantities

\footnotetext{
*Cominco Products Inc., Spokane, Washington.
} 
of Q-dope were used on the inner surfaces and a layer of Q-dope was applied on the outside so that the entire sample would be strengthened. The overall size of the resultant sandwich was $17 \mathrm{~mm} \times 10 \mathrm{~mm} \times 9 \mathrm{~mm}$. This procedure increased the surface area and cut down the eddy current losses. The r.f. coil was wound from No. 30 multistrand copper wire over a $0.025 \mathrm{~mm}$ thick mylar sheet on a form of the same size as the sample. The coil was than slipped over the sample for making measurements. The coil form made the coil rigid, reduced the possibility of straining the sample, and improved the $Q$ of the oscillator.

The sample used in this experiment is not the same as the one used previously. 1 However, it was cut from the same zonerefined bar which had a residual resistance ratio $\left(R_{300{ }_{K}} / R_{4.20 K}\right)$ $\approx 27,000$. It was found that the sample previously used was misoriented by approximately $10^{\circ}$ which accounted for the discrepancy between the observed periods and the periods shown in other effects.

All measurements were made at $1.2^{\circ} \mathrm{K}$ obtained by pumping on the $\mathrm{He}^{4}$ bath. The derivative of the r.f. absorption with respect to the field vs applied magnetic field was recorded using a standard PKW oscillator 15 and lock-in amplifier with field modulation. Data was recorded every $15 \mathrm{kc} / \mathrm{sec}$ between 16.5 and $25.9 \mathrm{mc} / \mathrm{sec}$ which covered a field range from $10.5 \mathrm{kG}$ to $16.2 \mathrm{~kg}$. The procedure during any given run was to set the oscillation frequency and sweep the magnetic field over a 5 gauss range centered on the expected resonant field. Each of the resonance 
curves obtained in this manner was then analyzed for the center field and changes in line shape. The line shape and width $(\sim 1.5$ gauss) remained constant within experimental error for all data reported here. 


\section{RESULTS}

Two sets of oscillations periodic in reciprocal field were observed in most of the range investigated. The longer period is dominant at fields around $11 \mathrm{kG}$ whereas the shorter period becomes dominant as the field is increased. In $F_{1,1} 1$ long and short period oscillations in different field ranges are shown from which a growth of the short period amplitude and a decay of long period amplitude is evident. The inverse field values versus integers for both periods are shown in Fig. 2. A detailed analysis of the amplitude of these oscillations was carried out over the entire range of the magnetic field and the overall profile shows a slight beat pattern. One reason for this is that the sample was not in one piece, but consisted of many single crystals joined together, thus every experimental point and curve represents an average from all of them. A point by point study of the amplitude was carried out at the maxima and minima of this envelope in several field ranges and the results are shown in Fig. 3. The crosses represent the amplitude at the points of maxima and the circles at the minima of the overall envelope. The average behavior is indicated by the dotted and continuous curves, respectively. The amplitude of the long period oscillations decreases as the magnetic field is 


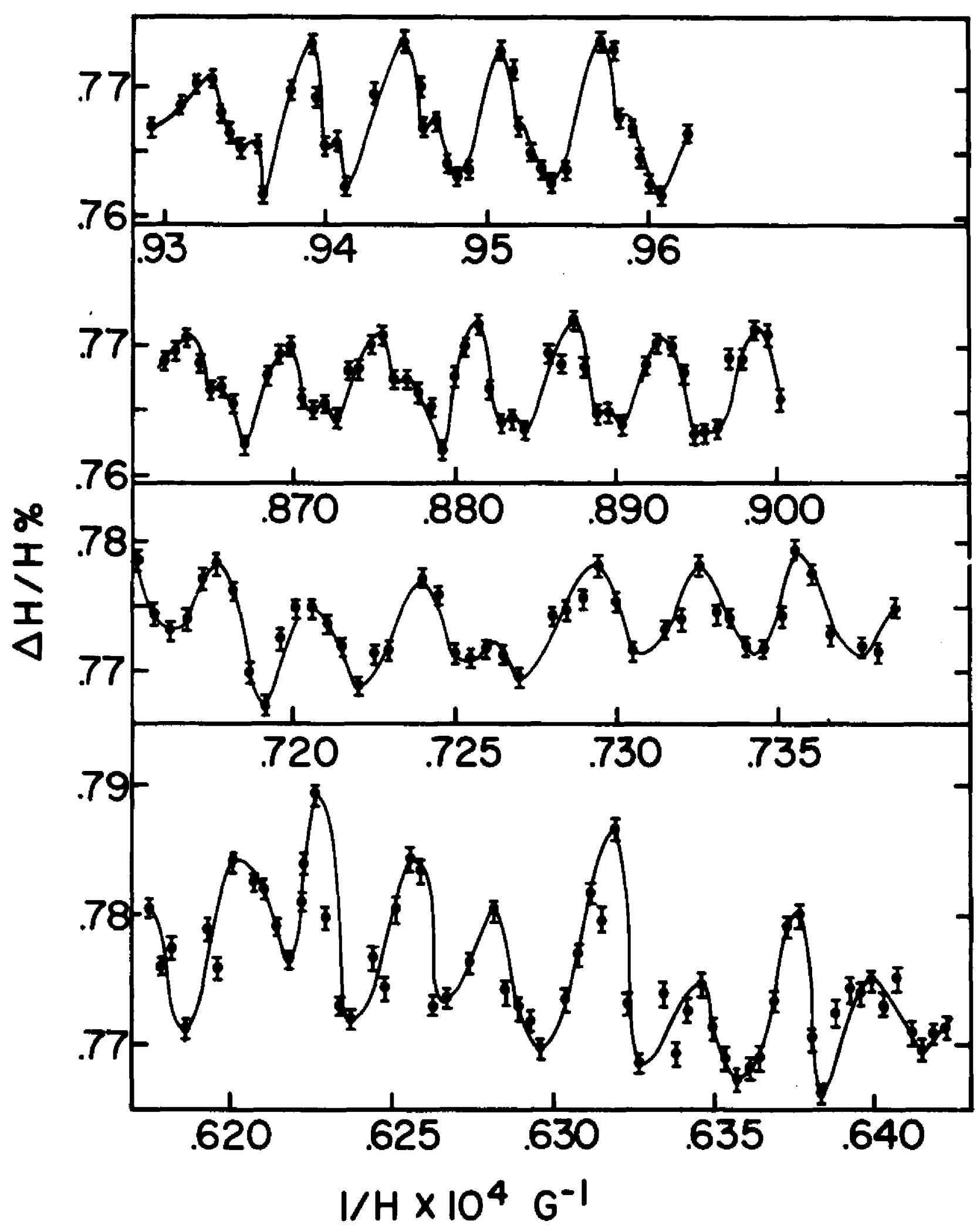

Fig. 1 


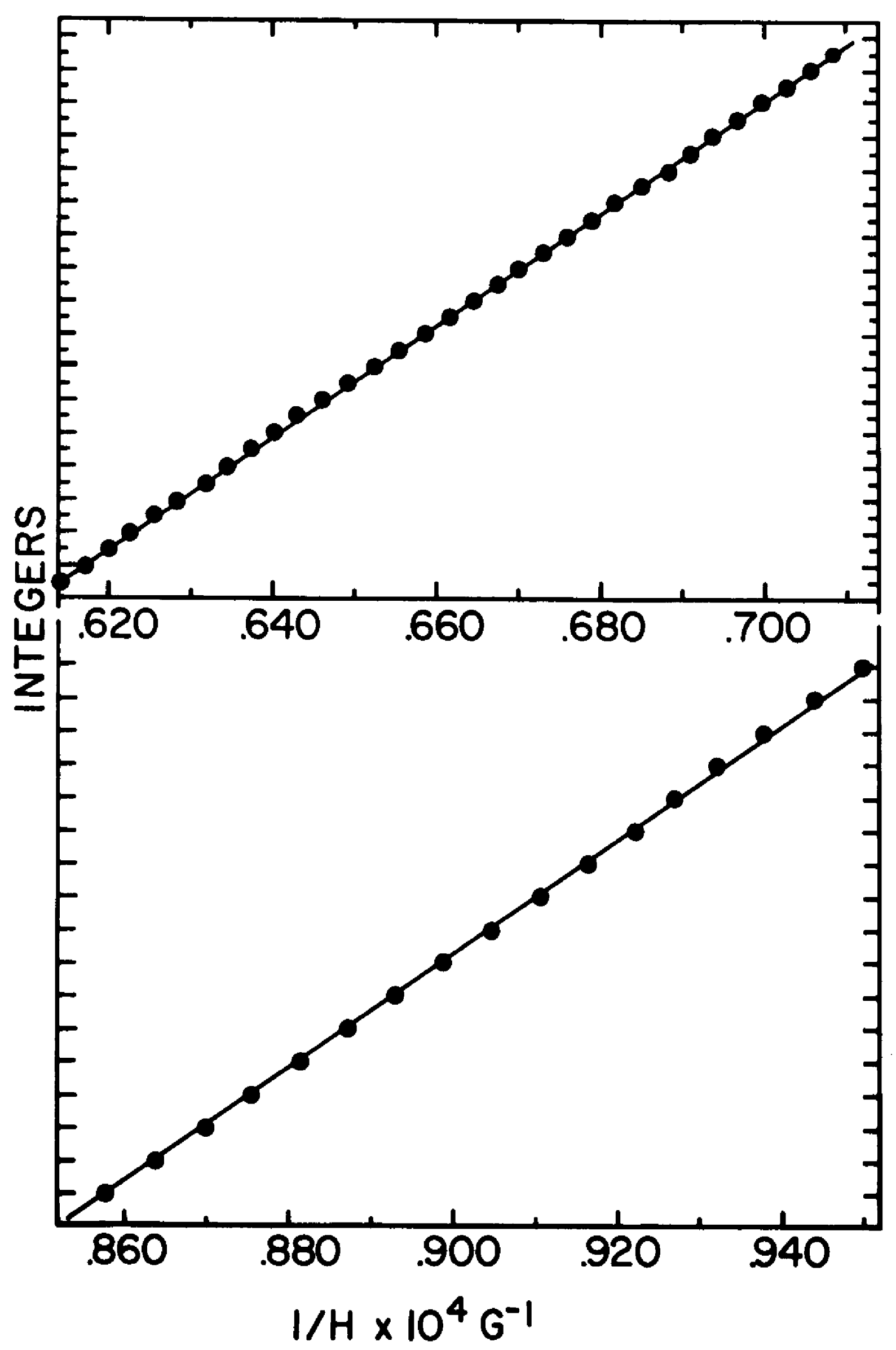

Fig. 2 


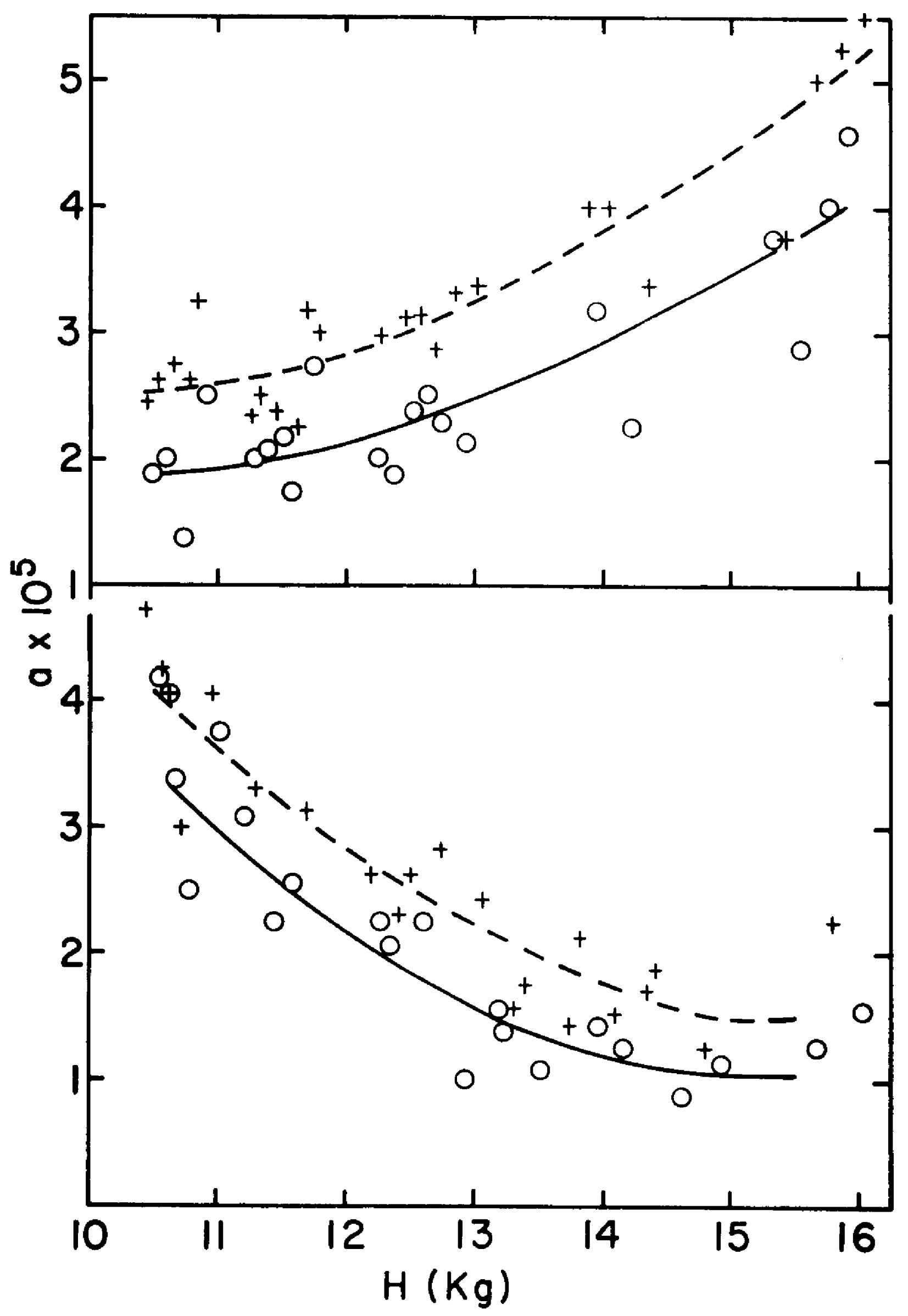

Fig. 3 
increased, whereas the short period oscillations show a continuous growth in amplitude with increasing magnetic field. Because of a wide scatter of these points no attempt has been made to $f i t$ these curves to a power law of the form $a=($ constant $) H^{ \pm n}$.

The angular dependence of period and amplitude were studied for both long and short period oscillations in different field ranges. The results of various runs are summarized in figs. 4 and 5. In Fig. 4 the dependence of the period on angle is depicted for both the short and the long period oscillations whereas in Fig. 5 the average dependence of the amplitude on angle for these oscillations is shown. It is evident from Fig. 4 that the long period decreases rapidly as the angle $\theta$ between the [001] direction and the magnetic field direction is increased. The short period shows a less rapid decrease. The linear fit of the square of the period of the long period oscillations versus $\cos ^{2} \theta$ (Fig. 6) indicates a cylindrical surface with axis along [001] giving rise to the long period oscillations. The average Knight shift displays an overall increase with increasing magnetic field as is shown in Fig. 7. This type behavior was also noted by Jones and Williams 14,15 in a lower range of magnetic field. Although the overall increase in $\sigma$ of $0.015 \%$ is evident in the extended field range of Fig. 7 , it was not a noticeable effect over the field range necessary for a few oscillations of the knight shift and, thus, was not taken into account in analyzing the amplitudes of the oscillatory components of $\sigma$. 


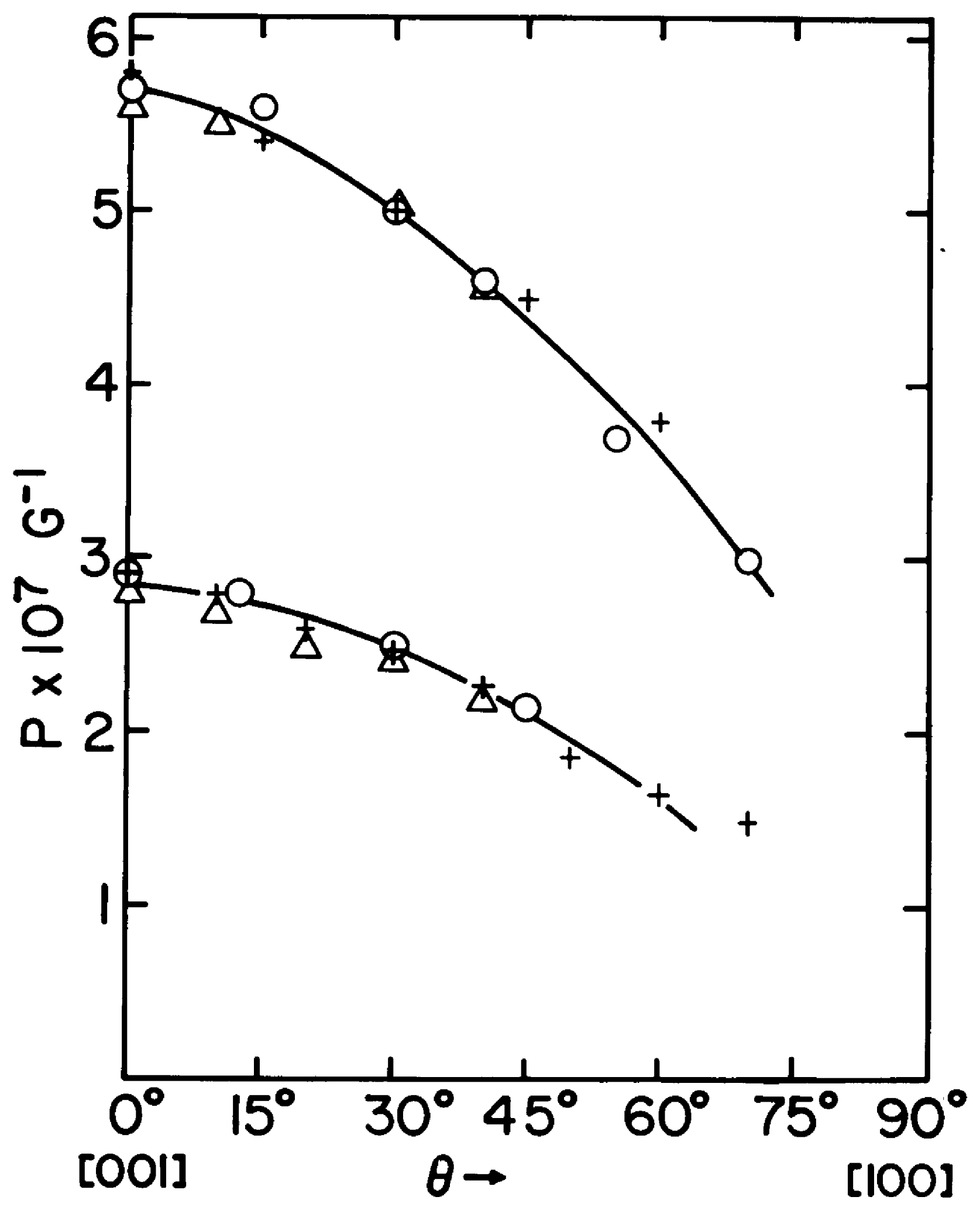

Fig. 4 


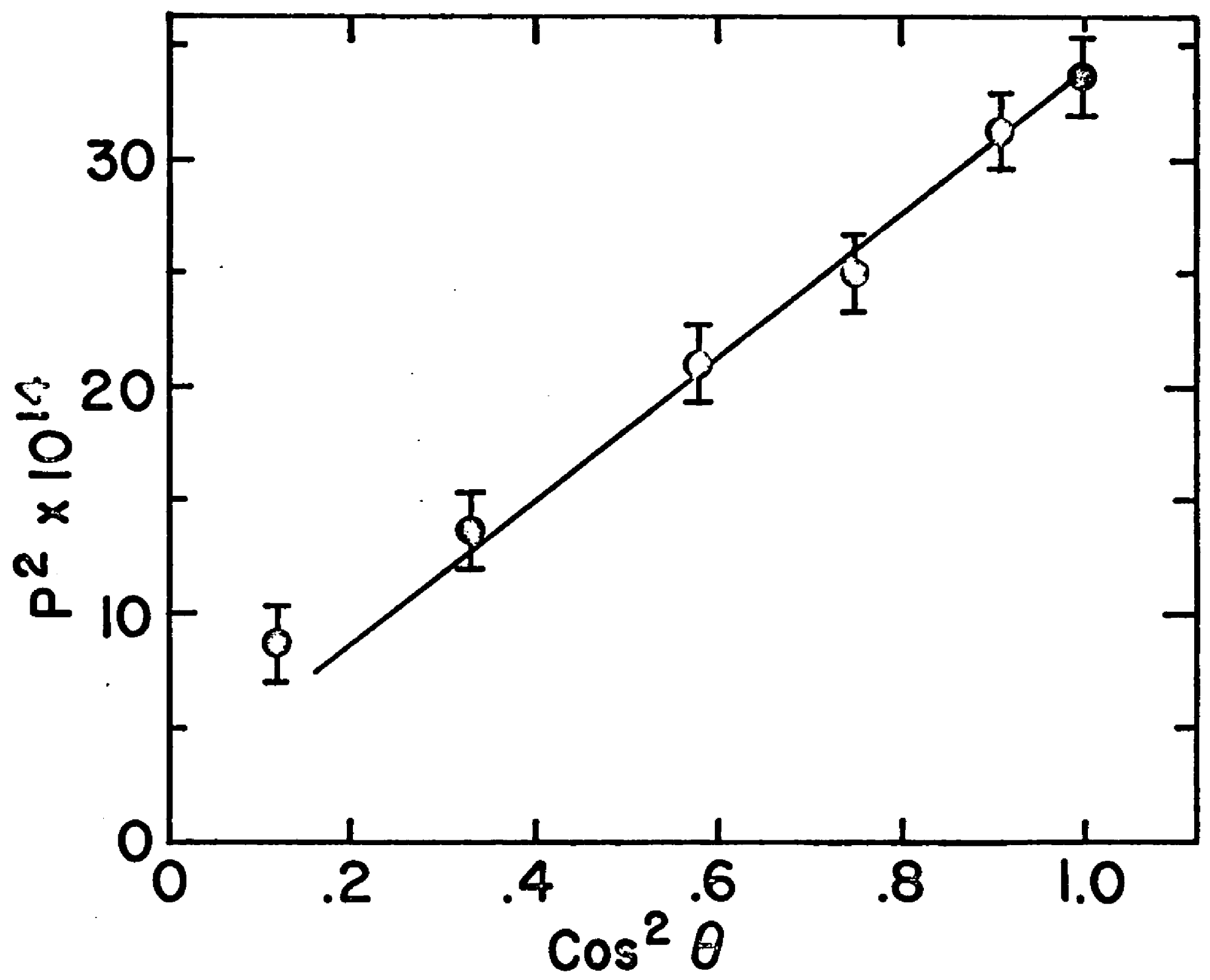




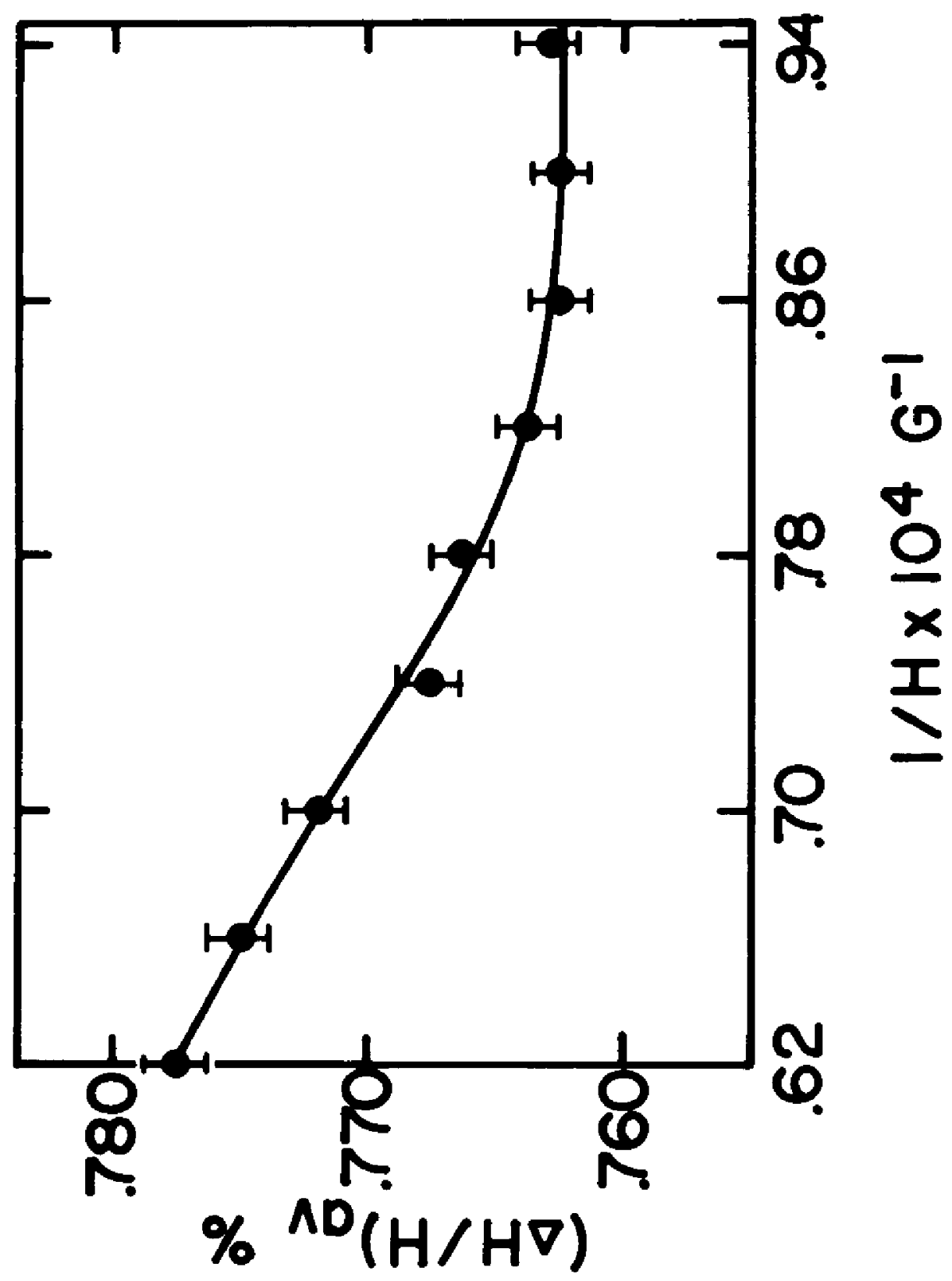

$\dot{r}$ 
For purposes of comparison, measurements of the de Haasvan Alphen oscillations in the magnetization were carried out on the same sample as that on which the Knight shift measurements were made. The field modulation technique ${ }^{16}$ was used and a signal proportional to the second derivative of the magnetization was recorded. A typical recorder trace is shown in Fig. 8. When detecting the second derivative of the magnetization oscillations the amplitude of the individual oscillations is attenuated by a factor of $\left(P_{i}^{2} H^{4}\right)^{-1}$, where $P_{i}$ is the period in reciprocal field of the $i^{\text {th }}$ oscillation. 17 To obtain the correct field dependence of the magnetization the amplitude must be multiplied by $\mathrm{P}_{\mathrm{i}}^{2} \mathrm{H}^{4}$ for each period. However, to compare this with the Knight shift measurements the field variation of the susceptibility $M / H$ must be obtained. This requires a point by point multiplication of the amplitudes by $\mathrm{P}_{i}^{2} \mathrm{H}^{3}$. The periods were analyzed by standard methods and their amplitudes plotted as a function of $\mathrm{H}$ after being multiplied by $\mathrm{P}_{i}^{2} \mathrm{H}^{3}$ and the results shown in Fig. 9 . 


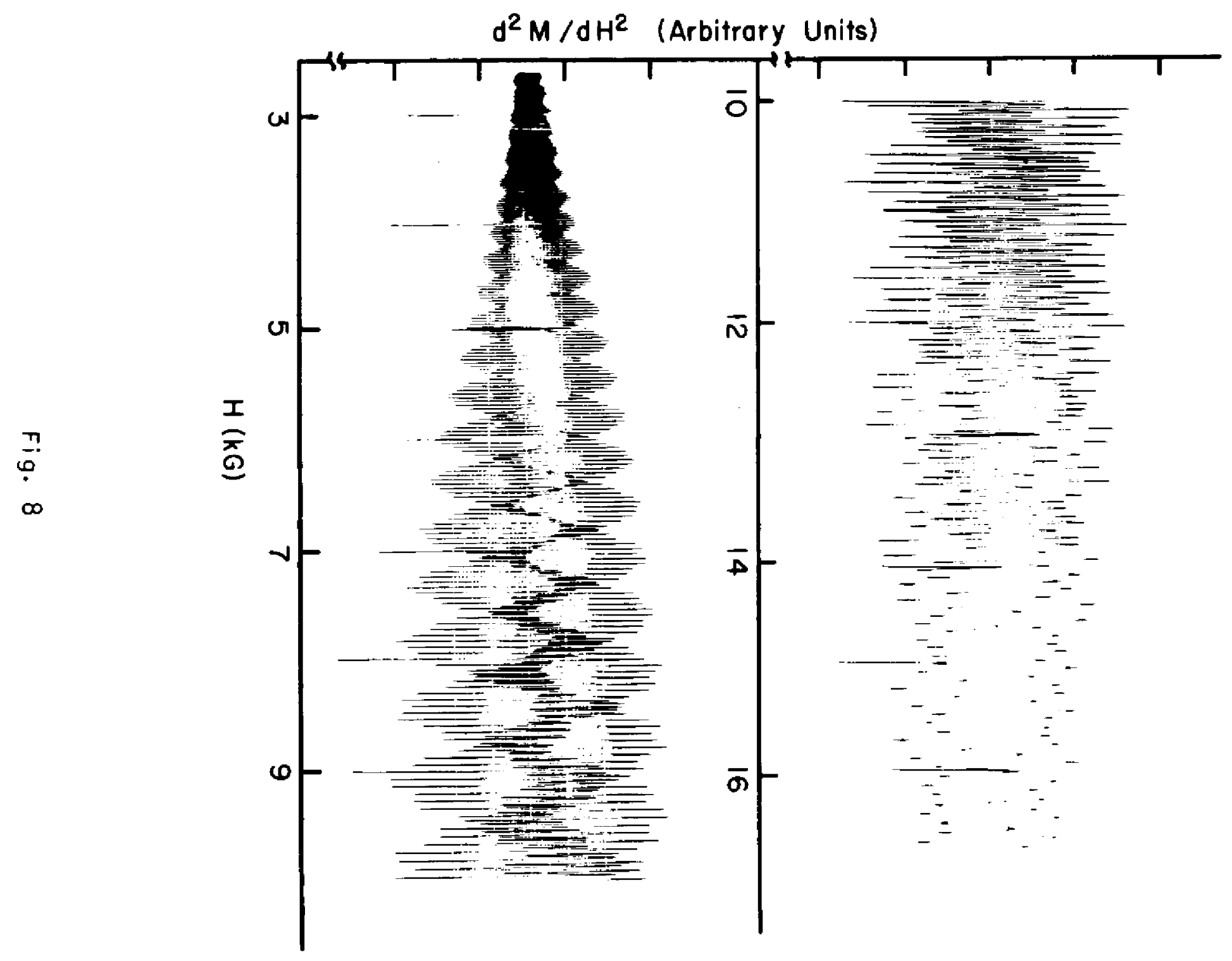




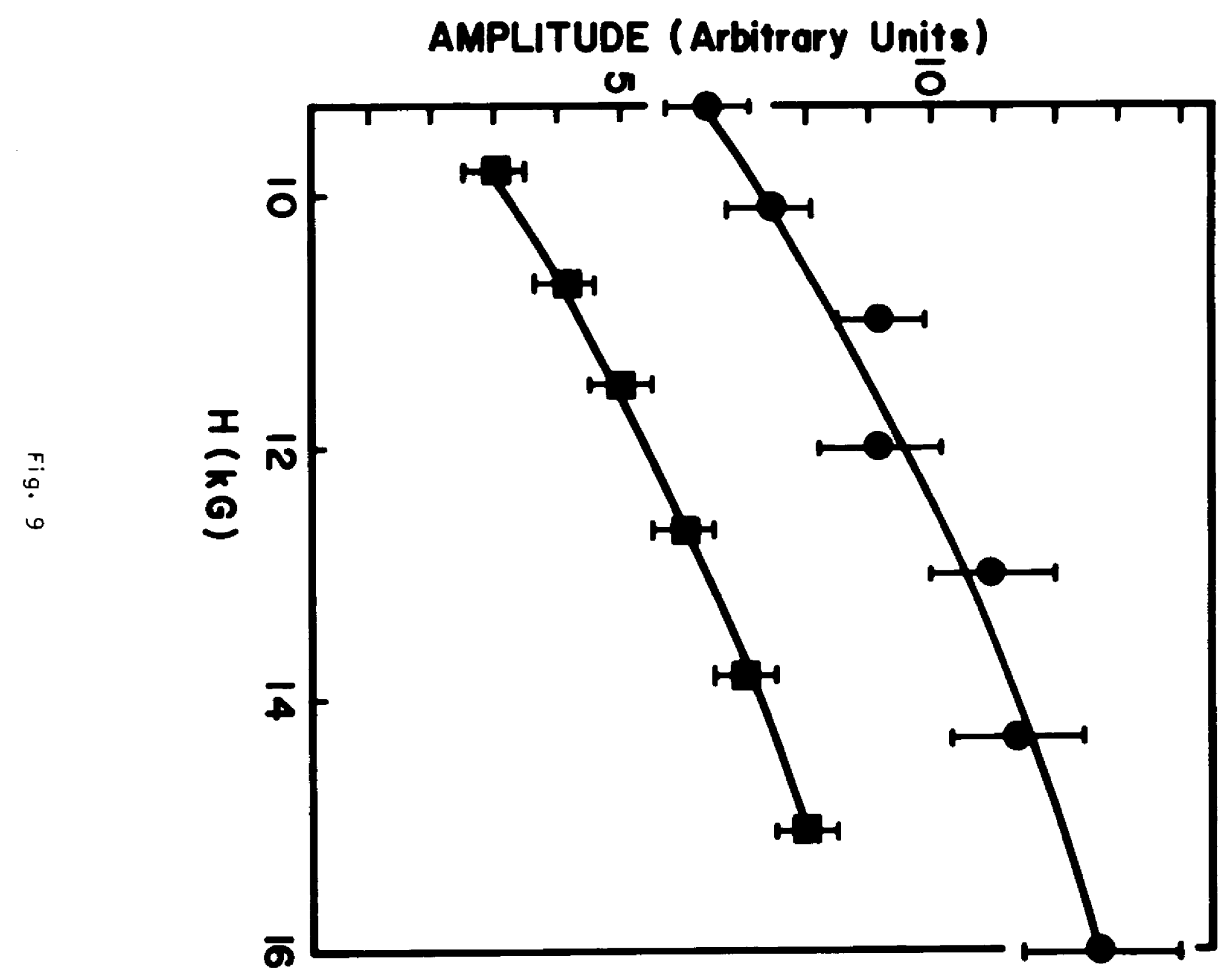




\section{DISCUSSION}

A. Comparison of Periods of Oscillations

with the Fermi Surface of Tin

In this section the experimental results of Section 111 are compared with the Fermi surface of $t i n .^{18}$ The two periods observed, $5.9 \times 10^{-7} \mathrm{G}^{-1}$ and $3 \times 10^{-7} \mathrm{G}^{-1}$ for the field along the $[001]$ direction, are in agreement with periods expected from the hole orbits $3^{\delta}{ }_{1}$ and $3_{2}{ }_{2}$, respectively. The $3_{2} \delta_{2}$ orbit is on the knobs of the third zone hole cylinders which appear according to the pseudopotential calculation of Weisz ${ }^{19}$ of the band structure of $s$ n and had previously been ascribed to the $5 \mu$ electron orbits existing in the nearly free electron model as given by Gold and Priestley. 18 The approximate cylindrical nature of the central portion of the third band hole surface defining the orbit $3^{6}{ }_{1}$ is apparent from the angular variation of the longer period shown in Fig. 4 and the $\mathrm{p}^{2}$ vs $\cos ^{2} \theta$ plot of Fig. 6. The angular variation of the shorter period is less rapid than the cosine (Fig. 4), suggesting a convex surface. This is consistent with the $3_{2}^{8}$ orbit on the knobs of the third zone cylinders. According to this interpretation, an amplitude maximum in the short period oscillations is to be expected at the magnetic field inclination where the ${ }^{6}{ }_{2}$ orbit on the knobs coincides*

* This maximum is probably due to a mixing of the $2^{\text {nd }}$ harmonic of one period with the fundamental frequency of the other. 
with the $3^{\delta}{ }_{1}$ orbit centered at the point $x$. As is shown in Fig. 5, an indication of this between $40^{\circ}$ and $50^{\circ}$ from the [001] direction is present. Thus, the periods obtained from the oscillatory Knight shift are in agreement with that expected from the Fermi surface of tin.

\section{B. Amplitude of the Oscillations}

In this section the observed amplitude of the oscillatory parts of the knight shift, $\widetilde{\sigma}$, are compared to the theoretical estimates of its components as calculated by various authors.

The most detailed calculations of the susceptibility contributions to the Knight shift was done by Stephen. 6 He calculated the exact free energy including the complete hyperfine interaction in the scaler effective mass approximation and obtained the following expressions for the paramagnetic and diamagnetic components of $\widetilde{\sigma}: *$

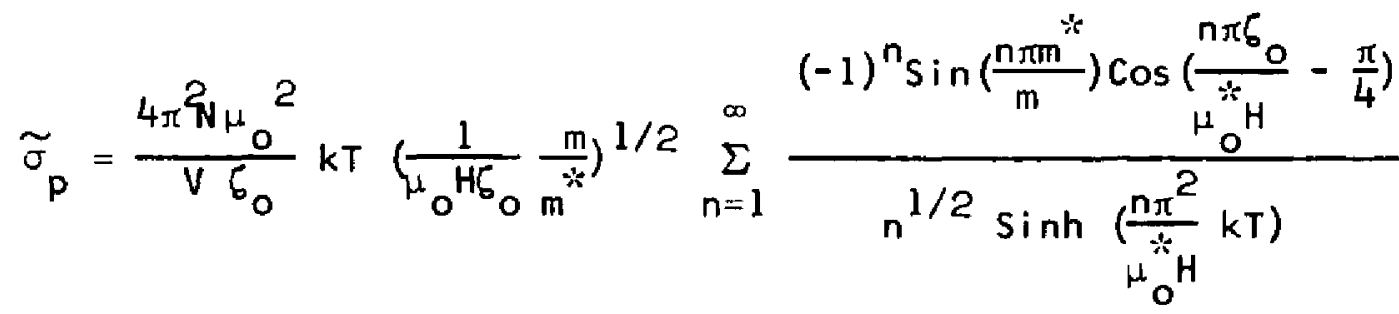

$$
\begin{aligned}
& \widetilde{\sigma}_{d}=-\frac{6 N \pi^{3} H_{o}^{*} k T}{V \zeta_{0} H} \sum_{n=1}^{\infty} \frac{(-1)^{n} I(n) \cos \left(\frac{n \pi m}{m}\right) \sin \left(\frac{n \pi \zeta_{0}}{\mu_{0}^{*} H}\right)}{\sinh \left(\frac{n \pi^{2}}{\mu_{0}^{*} H} k T\right)}
\end{aligned}
$$

*An integral occurring in Stephen's calculations of $\widetilde{\sigma}_{d}$ is in error by a factor of $(-2)$ and has been corrected here. For details see Ref. 20. 
wi th

$$
\sigma_{0}=\left(9 \pi \frac{N^{2}}{v^{2}}\right)^{1 / 3} \frac{\pi \hbar^{2}}{2 \pi^{*}}, \quad I(n)=\int_{0}^{1} d s[s(1-s)]^{1 / 2}|\sin (n \pi s)|
$$

where $\zeta_{0}$ is the Fermi energy, $k$ the Boltzmann constant, $T$ the absolute temperature, $m^{*}$ the effective mass of the carriers, $\mu_{0}^{*}$ the effective Bohr magneton, $N / V$ the density of the electrons taking part in the interaction, $I(n)$ an integral expression which has been evaluated numerically by Glasser, ${ }^{21}$ and $H$ the magnetic field. In the region of interest where $\pi^{2} k T / \mu_{0}^{*} H<1$, only the first terms of the sum in Eqs. (4) and (5) are important. In the liquid helium temperature range and in fields of $10^{4}$ gauss the amplitudes of the first terms are

$$
\begin{gathered}
\tilde{\sigma}_{p}=\frac{4 N}{v}\left(\frac{\mu_{0}}{\sigma_{0}}\right)^{3 / 2}\left(\frac{m}{m}\right)^{1 / 2} \mu_{0}^{*} H^{1 / 2} \\
\tilde{\sigma}_{d}=\frac{6 N \pi \mu_{0}^{* 2}}{V \sigma_{0}} \mid(1)
\end{gathered}
$$

where $l(1)=0.3$. Putting in values for the carriers observed in tin shows that in a magnetic field of $10^{4}$ gauss,

$$
\tilde{\sigma}_{p}=10^{-3} \tilde{\sigma}_{d}
$$

and, hence, only $\widetilde{\sigma}_{d}$ need be considered. In $\widetilde{\sigma}_{d}$ there is neither a field nor a temperature dependence of the amplitude. 
Dolgopolov and Bystrik ${ }^{7}$ retained only the Fermi contact part of the hyperfine interaction in the interaction Hamiltonian and obtained an expression for the oscillatory part of the Knight shift in terms of the local curvature of the Fermi surface $S$. Their result is

$$
\begin{gathered}
\tilde{\sigma}=\frac{8 \mu_{0}}{3 \pi \sqrt{2 \pi}}\left(\frac{e}{c \hbar}\right)^{3 / 2} \sqrt{H}\left|\frac{\partial^{2} S}{\partial p_{z}{ }^{2}}\right|_{m}^{-1 / 2} \\
\times \sum_{n=1}^{\infty} \frac{\psi(n \lambda)}{n^{3 / 2}} \cos \left[\frac{n c}{e^{\hbar H}} S_{m}\left(\zeta_{0}\right) \pm \frac{\pi}{4}-2 \pi n \gamma\right] \sin \left[\frac{n}{\partial m_{0}} \frac{\partial S\left(\zeta_{0}\right)}{\partial \zeta_{0}}\right.
\end{gathered}
$$

with

$$
\psi(n \lambda)=\frac{n \lambda}{\sinh n \lambda}, \quad \lambda=\frac{\pi c k T}{e^{\hbar H}} \frac{\partial S_{m}(\epsilon)}{\partial \epsilon}
$$

where $S_{m}$ is the area of an extremal section of the Fermi surface. It is seen that the amplitude increases as $H^{1 / 2}$ in this expression. A numerical estimate of the amplitude is obtained by comparing the above expression with the oscillating part of the magnetic susceptibility $x_{p} \cdot 22$ This gives

$$
\widetilde{\sigma}=\frac{8 \pi \mu_{\mathrm{o}}}{3} \frac{\partial S_{\mathrm{m}}(\zeta)}{\partial \zeta} \frac{1}{S_{m}(\zeta)} \times{ }_{p} H
$$

Assuming the wave function factor to be unity and constant,

$$
\sigma_{p}=\frac{8}{3} \pi x_{p}
$$


thus

$$
\begin{aligned}
\widetilde{\sigma} & =\mu_{0} \frac{\partial S_{m}(\zeta)}{\partial \zeta} \frac{1}{S_{m}(\zeta)} \sigma_{p} H \\
& \simeq \mu_{0} \frac{H}{\zeta} \sigma_{p} .
\end{aligned}
$$

For tin in a field of $10^{4}$ gauss the largest this can be is $\widetilde{\sigma}=10^{-7}$. For a parabolic band their result agrees with Stephen's $\sigma_{p}$

In the nearly free electron approximation, Glasser ${ }^{9}$ obtains an expression for the oscillatory part of the Knight shift by expanding the free energy, in powers of the lattice potential $V(\underline{r})=\Sigma_{K} V_{K} e^{i \underline{K} \cdot \underline{r}}$ where $\underline{K}$ is a reciprocal lattice vector. The validity of this calculation is limited to two regions of magnetic field: (1) weak fields where $\mu_{0} H \ll v_{K}, \zeta \gg v_{K^{\prime}}$ (2) high fields where $\mu_{\mathrm{O}} H \gg V_{K}, \zeta \gg V_{K}$, and $K \neq 2 K_{F}$ where $K_{F}$ is the radius of the free electron sphere. Oscillatory behavior is obtained only in the high field limit with the result:

$$
\begin{aligned}
& \tilde{\sigma}=\frac{1}{2} \sqrt{\mu_{o}{ }^{H}}\left[\frac { 8 \pi } { 3 } \mu _ { o } ^ { 2 } ( \frac { m } { 2 \pi \hbar ^ { 2 } } ) ^ { 3 / 2 } \sum _ { K } \frac { V _ { K } } { 6 } \left[s_{o}^{-3 / 2} \cos \left(\frac{\pi \xi_{o}}{\mu_{0}^{H}}+\frac{\pi}{4}\right)\right.\right. \\
& \left.-\sum_{k=1}^{\infty}\left\{\frac{(k+1)}{\left|k \pi+s_{0}\right|^{3 / 2}} \cos \left(\frac{\pi \xi_{k}^{+}}{\mu_{0} H}+\frac{\pi}{4}\right)+\frac{(k-1)}{\left|k \pi-s_{0}\right|^{3 / 2}} \cos \left(\frac{\pi \xi_{k}^{-}}{\mu_{0}^{H}}+\frac{\pi}{4}\right)\right\}\right]
\end{aligned}
$$

Where $\xi_{0}=\frac{1}{2 \pi} \in(\underline{k}) g_{1}\left(S_{0}\right), \xi_{k}^{ \pm}=k \zeta \pm \xi_{o}, \epsilon(\underline{k})=\frac{\hbar^{2}}{2 m} k_{z}^{2}+\frac{\hbar^{2}}{2 m}\left(k_{x}^{2}+k_{y}^{2}\right)$ $g_{1}\left(s_{0}\right)=\frac{1}{2}$ a $s_{0}-(a-1)^{1 / 2}, a=\frac{4 \zeta}{\epsilon(\underline{k})}$ and $\cos s_{0}=\frac{2-a}{a}$ 
Thus the amplitude of the oscillations increases as $H^{1 / 2}$. The result is applied to the case of an orbit about a symmetry point on the zone boundary and the amplitude of the oscillations is

$$
r=\frac{\text { amp } \tilde{\sigma}}{\sigma(H=0)}=\frac{\text { amp } \tilde{\sigma}}{\frac{8 \pi}{3} x_{p}}=\frac{\left(\pi \mu_{o} H\right)^{1 / 2} \delta}{8\left(\zeta s_{o}\right)^{3 / 2}}
$$

where $\delta$ is a band gap and $S_{0}$ is the angular aperture of the particular sheet of the Fermi surface as seen from the center of the zone. For a value of $S_{0}$ of a few degrees this expression leads to an amplitude of the observed magnitude.

The numerical results of all the calculations are summarized in Table 1. Glasser's calculations give the best agreement with the observed value of the amplitude at $10^{4}$ gauss for the case of tin. Stephen's estimates based on diamagnetic effects in the magnetic susceptibility are almost an order of magnitude too small.

Since both the diamagnetic susceptibility factor and the wave function factor give estimates of the amplitude which are within an order of magnitude of the observed amplitude, the field dependence of the amplitude must be considered to find the major contributing component. The theories of Glasser ${ }^{9}$ and Dolgopolov and Bystrik ${ }^{7}$ predict a $\mathrm{H}^{1 / 2}$ field dependence for the oscillation amplitude under normal conditions, i.e., not considering the effect of magnetic breakdown. In Stephen's calculation the paramagnetic component is three orders of magnitude 
TABLE I

Theory

Amp l i tude

ratio $\frac{\text { calculated }}{\text { observed }}$

1. Stephen ${ }^{a}$

$6.8 \times 10^{-6}$

$\sim \frac{1}{7}$

2. Dolgopolov and Bystrik

$\sim 10^{-7}$

$\sim \frac{1}{400}$

3. Glasser ${ }^{c}$

$4 \times 10^{-5}$

$\sim 1$
a. See $\operatorname{Ref} .6$.
b. See Ref. 7 .
c. See Ref. 9 . 
smaller than the diamagnetic component, and has a field dependence of $H^{1 / 2}$ and the more dominant diamagnetic oscillations have no field dependence. Experimentally, however, the amplitude is found to depend on the magnetic field. In the case of the short period oscillation $\left(3 \times 10^{-7} \mathrm{G}^{-1}\right)$ the amplitude increases with the magnetic field. This rules out the possibility of the oscillations being due to oscillations in the diamagnetic susceptibility. However, a diamagnetic contribution to the wave function factor has not been examined. Further support to the argument that the Knight shift oscillations do not arise from the susceptibility terms is found from the magnetization measurements. As can be seen from a comparison of Figs. 3 and 9 the field dependences of the amplitudes of $\widetilde{\sigma}$ and $\tilde{x}$ are in opposite directions for the $3{ }_{1}$ orbit and of a different power for the $3_{2}^{\delta}$ orbit.

One effect that has not been included in any of the above calculations is that of spin splitting of the Landau levels in a magnetic field. $*$ For the field directed along the $[001]$ axis there are two neighboring orbits in the same plane, $3^{\delta}{ }_{1}$ and $4 a \zeta$. On the fourth band the $\delta$ orbit is quite extended and the electrons do not remain particularly near any symmetry point in the zone, thus one does not expect these electrons to be characterized by any particular $s$ or $p$ character near the atomic sites. However, on the $3_{1}^{\delta}$ orbit the electrons remain near the symmetry point $X$ and retain a large amount of s character. In the presence of

*The author is indebted to W. G. Chambers for suggesting this mechanism. 
a strong magnetic field the Landau levels may be split into spin up and spin down states causing an asymetric distribution of the two spin states at the Fermi level. This type energy level distribution is shown in Fig. 10 for the ${ }^{3 \delta} 1$ orbit having a large spin splitting and for the $4 \zeta$ orbit with a larger effective mass and, thus, smaller spin splitting. Since the wave functions of the electrons on the $3 \delta$ orbit have a large component of $s$ character they should exert a large influence on the contact term of the hyperfine interaction and as the magnetic field increases and the different spin orientations pass through the Fermi level this influence will completely reverse sign at the periodicity of the de Has-van Alphen period for the $3^{\delta}{ }_{1}$ orbit. This effect would not present a large distortion in the magnetization oscillations since the major contribution in these is of a diamagnetic origin.

\section{Effect of Magnetic Breakdown}

The decrease in amplitude of the component of $\widetilde{\sigma}$ having the longer period $\left(3^{\delta}{ }_{1}\right.$ orbit) with increasing magnetic field is attributed to the effect of magnetic breakdown. ${ }^{8}$ in the absence of any spin-orbit coupling the symmetry of the white tin lattice requires the existence of degenerate energy bands along the XL and XP 1 ines on the $\langle 110\rangle$ face of the Brillouin zone. $13,23,24$ This degeneracy is preserved only at the points $X$ and $L$ when spin-orbit effects are taken into account, leaving a very small 
band gap between the third and fourth bands near the point $x$. Thus, magnetic breakdown occurs at moderate field strengths and couples the $3^{\delta}{ }_{1}$ orbit on the third band to the $4 a b$ orbit on the fourth band. The effect of magnetic breakdown manifests itself quite differently on $\tilde{\sigma}$ from $i$ ts effect on the susceptibility oscillations. Assuming, then, that the Knight shift oscillations are indeed due to oscillations in the ground state wave functions, this behavior indicates that the wave functions are much more sensitive to the amount of tunneling occurring during breakdown than are the susceptibility oscillations. This is compatible with the spinsplitting argument presented in the previous section in which the oscillation amplitude is very sensitive to the wave functions having a definite symetry. Breakdown would cause the $3{ }^{6}{ }_{1}$ electrons to lose this symmetry as they tunnel into the $4 a b$ orbit. 


\section{CONCLUSION}

None of the theories of $\widetilde{\sigma}$ advanced so far completely explain the behavior of the Knight shift as a function of applied field in Sn. Glasser ${ }^{9}$ has obtained an expression for the amplitude of $\tilde{\sigma}$ which depends on oscillations in the ground state wave functions of the contributing electrons that gives the correct amplitude at moderate fields. However, his calculation does not include magnetic breakdown effects which tend to completely dampen the oscillations in a rather narrow field range. For the observed orbit that does not experience breakdown effects the observed field dependence of $\tilde{\sigma}$ differs from that predicted by Glasser, ${ }^{9}$ but nevertheless increases in amplitude with increased field. Measurements of the de Haas-van Alphen effect on the same sample as the Knight shift measurements were performed rule out the possibility of $\tilde{\sigma}$ simply being a result of magnetization oscillations in the sample. 


\section{APPENDIX I}

\section{Additional Experimental Details}

\section{A. General Considerations:}

The radio-frequency magnetic field which is used to excite the resonance in the nuclear system induces eddy currents in the sample which reduce the penetration into the sample to a depth of the order of the skin layer. This makes the study of NMR in metals difficult in two ways when the dimensions of the sample are large in comparison with the radio frequency skin depth: (1) It reduces the working volume of the substance, thereby reducing the total number of spins participating in the resonance. (2) It makes the resonance absorption depend not only on the imaginary part, $\chi^{\prime \prime}$, of the dynamic susceptibility $X=X^{\prime}-i X^{\prime \prime}$ as is the case with non-metallic compounds, but also on its real part $X^{\prime}$. In fact the quantity $X^{\prime}$ determines the skin depth; as $X^{\prime}$ increases the skin depth is reduced, thus reducing the absorption of energy in the sample. Experimentally the extent to which $x^{\prime}$ determines the absorption is manifest in a distortion of the shape of the absorption line. The most readily observable measure of the distortion of the line is the ratio of the amplitudes of the extrema of the derivative of the absorption line. In order to minimize these effects the usual practice is to make the sample either in powder form or in the form of thin foils insulated 
from each other so that at least one dimension of the specimen is smaller than the radio frequency skin depth. This procedure gives a substantial filling factor and makes absorption proportional only to $X^{\prime \prime}$. For experiments with single crystals it is not possible to obtain the sample in either the powder or the foil form. Instead, satisfactory results are obtained by preparing the single crystal sample in the form of thin wafers and sandwiching them together with thin layers of insulating material between them.

Although the thickness of the wafers in the tin sample was much greater than the radio frequency skin depth, only a small distortion of the NMR signal was observed in the actual experiments. This is in agreement with the findings of Redfield 25 in aluminum and copper, of Karimov and Shchegolev ${ }^{12}$ in powdered tin, and of Jones and Williams ${ }^{14}$ in a tin single crystal. It was noted by Karimov and Shchegolev ${ }^{12}$ that for resonant circuits of low $Q$ the contribution of $X^{\prime}$ to the observed absorption may be quite small. This is probably the case with metals when the coil of the oscillator tank circuit is wound on the specimen itself. Another reason for this observed discrepancy from the predictions of the classical theory may be, as has been noted by Allen and Seymour, ${ }^{26}$ that anomalous skin effect conditions prevail partially or wholly at low temperatures where the penetration depth of the radio frequency field is comparable with the mean free path of the conduction electrons. Classical predictions of absorption signals which are too small and distortion which is too large do not seem to be applicable in the NMR experiments at low temperatures. 
In other words, the actual NMR skin depth seems to be much larger than the one predicted by classical theory.

In order to take into account the shape of the sample we must consider the demagnetization factor $D$ which can have any value between zero and one. This has been done by Yafet ${ }^{27}$ and Hebborn ${ }^{28}$ who write the shielding constant in the form

$$
\sigma=4 \pi(1-D) x
$$

where $X$ is the diamagnetic susceptibility. In order to include the demagnetization effects in the oscillatory knight shift one has to multiply by $(1-D)$ the amplitude factor of the calculated diamagnetic term. The geometry of the wafers was chosen in such a way that $D$ was very small when the magnetic field was parallel to the symmetry axis of the crystal. However, when the wafers are sandwiched together with insulating material between them, the demagnetization factor certainly increases. Anyway, even if we regard the whole sample as a single metal block, (even though it may not be right to do so since the coupling between the various wafers cannot be perfect), the geometry of the sample was such than $D$ was about $1 / 2$. To make $D$ smaller, the number of wafers which are stacked together should be smaller but it would result in a small filling factor giving a smaller signal to noise ratio and reducing the sensitivity of observation. The sample geometry was a good compromise between $D$ and the sample filling factor. Moreover, it was considered, while deciding on the sample geometry, that $D$ does not change much when the magnetic field is rotated around to make angular studies. 


\section{B. Description of Equipment:}

The detecting circuit was a Pound-Knight-Watkins ${ }^{15}$ marginal oscillator with high $Q$ voltage sensitive diodes substituted for the mechanically variable capacitor in the tank circuit of the oscillator. This was done to minimize incidental noise and to allow frequency variation to be voltage controlled. The $Q$ of the varicaps used was between 200 and 300 . The voltage input to the varicaps was obtained from a modified Tektronix ${ }^{29}$ Type 162 waveform generator. ${ }^{30}$ In Fig. (11) is shown the circuit diagram of the power supply which was used to feed the waveform generator. A circuit diagram of the complete radio frequency oscillator is given in Fig. (12). A high pass filter network ${ }^{31}$ was introduced in the sample coil circuit of the oscillator in order to cut down inductive pickup from the modulation coils which were mounted directly over the pole-pieces of the magnet. This addition allowed high gain to be used in the audio amplification stages without saturating the amplifiers. The automatic gain control circuit of the oscillator was modified ${ }^{32}$ and low noise tubes were substituted in the r.f. and audio amplification stages. General purpose germanium diodes were substituted for electronic tube diodes. Germanium diodes as detectors, while noisier than vacuum tubes, do not introduce spurious and variable dc output. To minimize microphonic pickup and r.f. leakage in the circuit, the oscillator, r.f. amplifier, detector and audio amplifier stages were built in well shielded compartments of the chasis and lead lengths were reduced to a minimum. All this carefull attention 


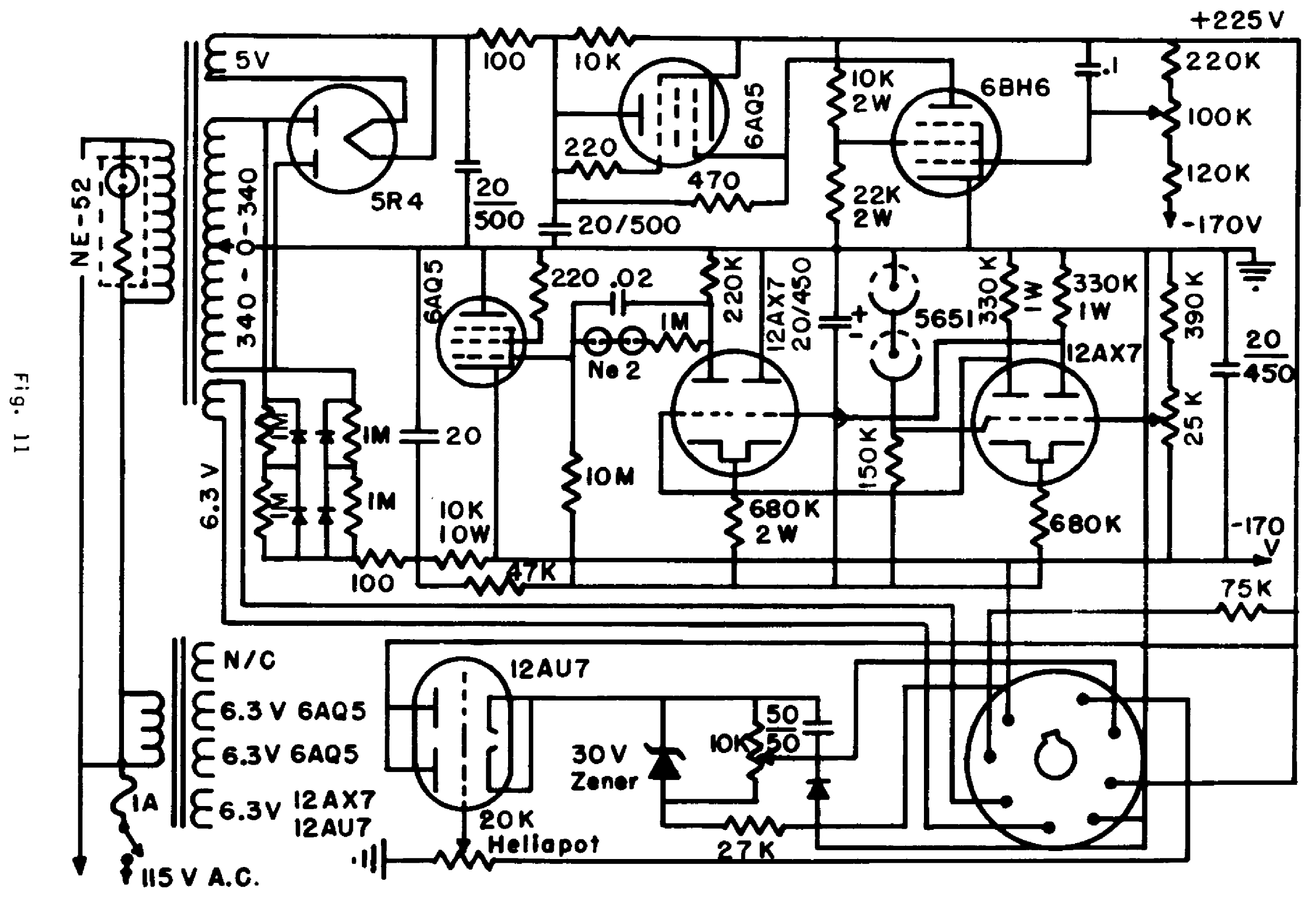




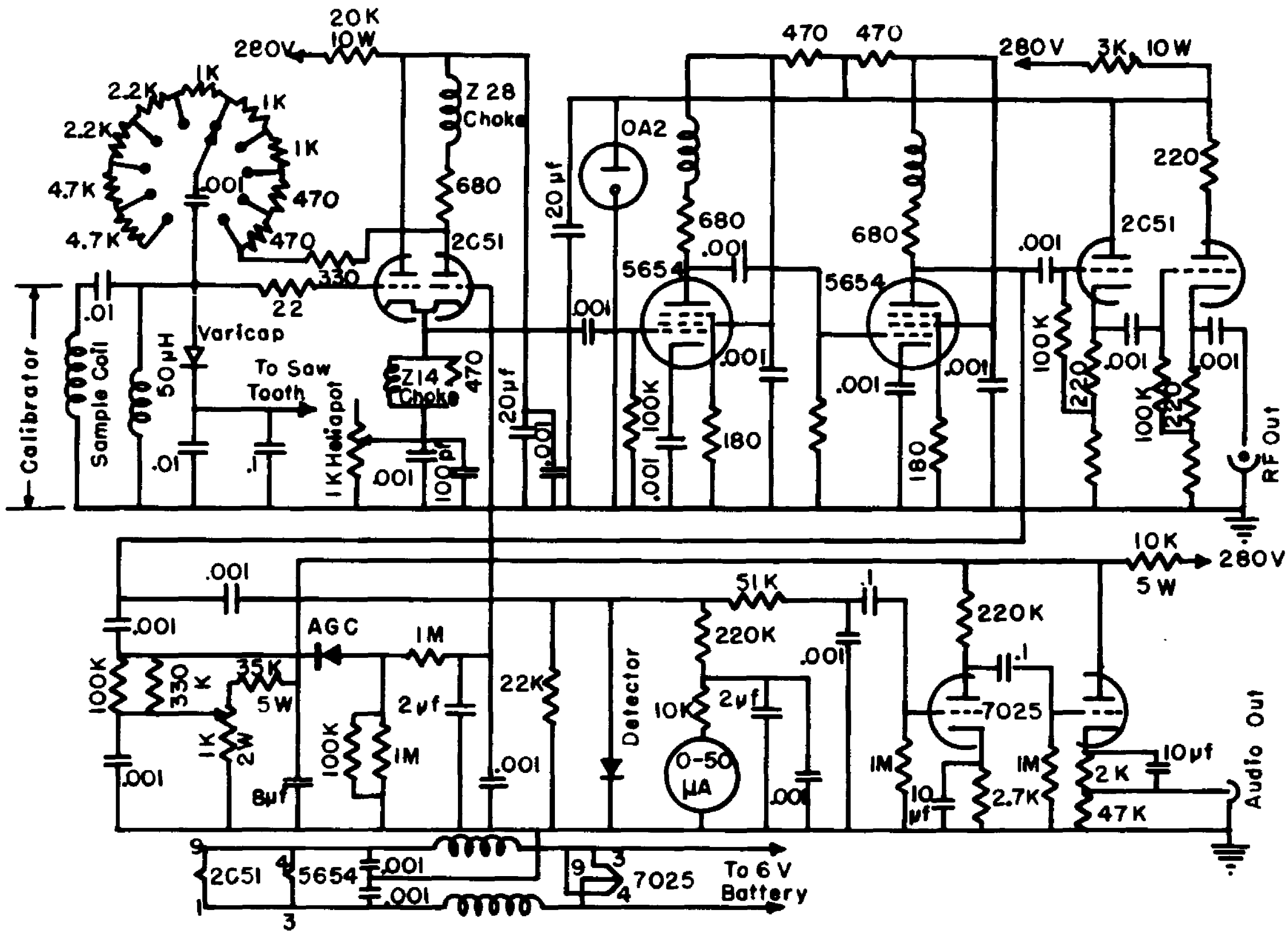


to layout, shielding, decoupling, and rigid construction resulted in a stable operation of the oscillator. Care was taken not to introduce any ground loops and the entire circuit was grounded at only one point of the chasis. The heater current to the vacuum tubes was supplied by freshly charged $6 \mathrm{~V}$ batteries and low-pass filters were inserted in each of the two leads from the battery. All of these precautions resulted in quite good sensitivity for a wide range of r.f. voltage. The sensitivity of the oscillator versus r.f. amplitude was checked with a calibrator circuit of Watkins ${ }^{14}$ which is shown in Fig. (13) after the sample was in place, but the calibrator was disconnected during the course of the measurements. It was found that better sensitivity could be obtained in the NMR measurements using the two largest feedback resistor at which r.f. oscillations could be smoothly maintained without any motorboating occurring. The signal to noise ratio was found to depend on the r.f. level at which the oscillator operated. The r.f. level was adjusted for maximum signal to noise ratio during the course of the measurements.

With some modifications in the feedback circuit, the marginal oscillator has been used to detect quantum oscillations of the high frequency surface resistance ${ }^{33}$ at relatively low magnetic fields. Some changes are needed in the circuit for the stabilization and regulation of the amplitude which, as a consequence, result in an increase in the sensitivity of the threshold oscillator. Strong feedback is required to maintain the oscillation amplitude over a wide range of the magnetic field. For this purpose a 


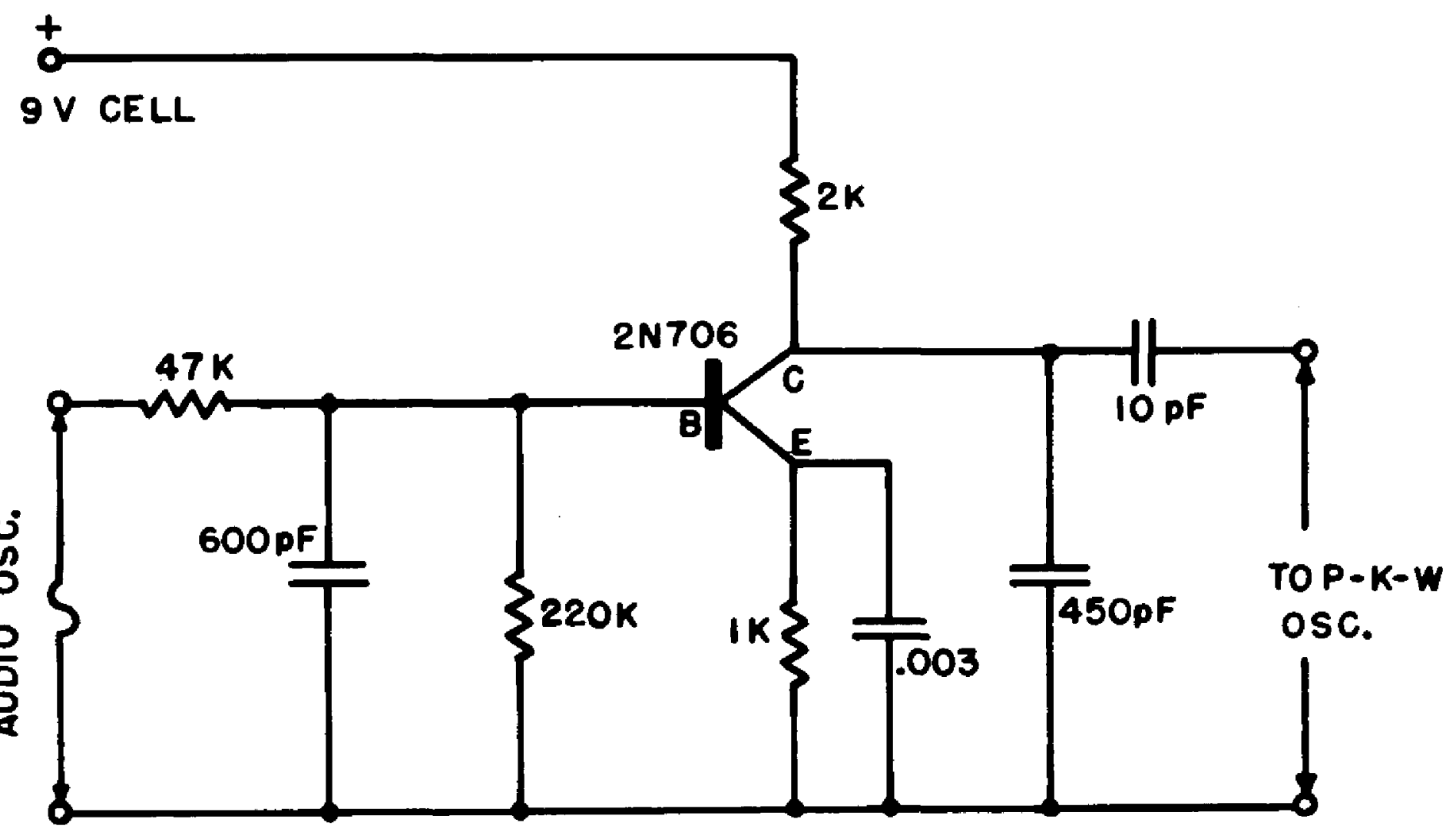


standard amplifier can be introduced in the feedback network. 34

In order to see if the NMR circuit used in the present experiment was in any way sensitive to the surface impedance oscillations, the magnetic field was swept over a range of 500 gauss around a field value of about 12.0 kilogauss and about 200 gauss at 13 ki logauss at liquid helium temperatures. No signs of such an effect were observed within the accuracy of our measurements. Thus there was no possibility of the center of the NMR line being influenced by the surface impedance oscillations so as to give rise to a spurious effect which had nothing to do with the real Knight shift.

The sample was attached to a sample mounting assembly at the end of a $1 / 2$ inch o.d. stainless steel tube extending into the helium dewar. The sample mounting assembly is shown in Fig. (14). A number 28 copper wire was passed through the tube coaxially and was held rigidly in position by teflon spacers and thin ceramic sleeves. This assembly formed the transmission line connecting the tank coil to the remainder of the oscillator and also served as a support for the sample in the dewar. All mounting was done to minimize the stray capacitance while keeping the $Q$ of the circuit as high as possible. The oscillator was mounted at the top of the sample holder and a rigid connection was used to connect the r.f. line to the oscillator. The base of the oscillator chasis was made heavy and was shock mounted to avoid mechanical vibrations causing microphonic pickup.

In Fig. (15) is shown a block diagram of the entire measuring system. The constant d.c. magnetic field was modulated at a 


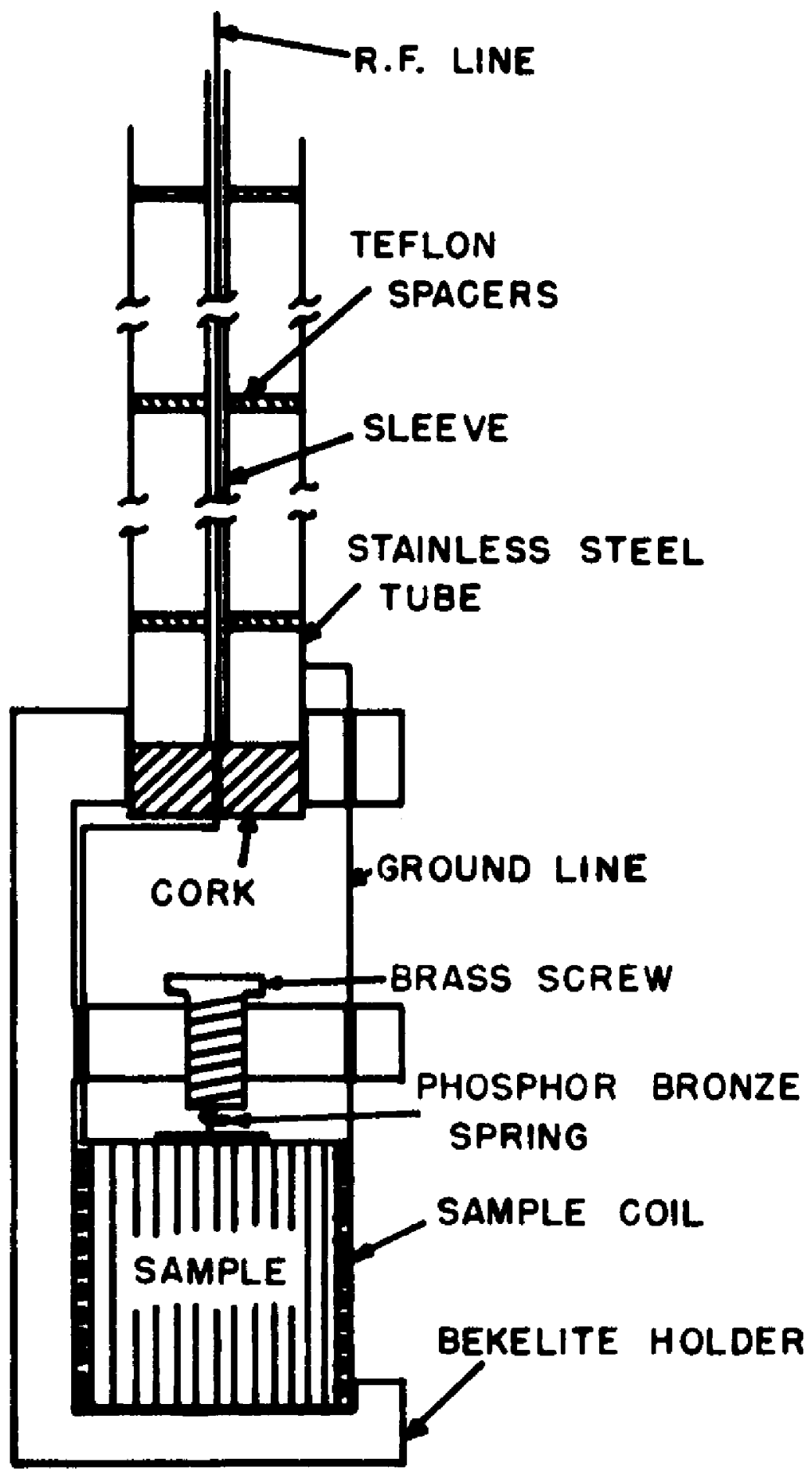

Fig. 14 
frequency of $300 \mathrm{cps}$ with an amplitude of about one-third of a gauss peak to peak. This was ascertained by a standard pickup coil inserted at the position of the sample. The modulation coils were fed by a Hewlett-Packard ${ }^{29}$ audio oscillator through a McIntosh 75 power amplifier. 29 The audio output of the r.f. oscillator was fed to a phase-sensitive detector of standard design ${ }^{29}$ (EMC - Model RJB). To have proper line shape the total time to sweep through the NMR line was kept several times the time constant used in the lock-in-amplifier. The phase-sensitive detector fed a Moseley $X-Y$ recorder ${ }^{29}$ which plotted the derivative with respect to the magnetic field of the NMR absorption line on the $Y$-axis, the $X$-axis being driven by a voltage from the magnet sweep unit which was proportional to the swept-over magnetic field. The frequency of the oscillator was measured using a Hewlett-Packard model $5245 \mathrm{~L}$ Electronic counter. 29 The magnet used in the experiment was a Magnion Model L-158, 15" laboratory electromagnet. 29 It was capable of giving a field up to 26 kilogauss. To obtain a good magnetic field stability the special features of the magnet were a stationary field-rate sensing coil and a rotating coil field sensor. The short and long term magnetic field stabilities were respectively one milligauss ( 1 to 100 seconds) and one part in $10^{6}$ of the operating field ( 100 seconds to 60 minutes).

C. Data Recording and Analys is:

The magnetic field was calibrated in terms of the $\mathrm{Sn}^{119}$ resonance in a saturated solution of $\mathrm{SnCl}_{2}$ in concentrated $\mathrm{HCl}$, with a 0.1 molar addition of $\mathrm{MnCl}_{2}$. To obtain the metal resonance, 
the approximate position of the absorption line was calculated from the known position of the resonance line in the salt solution and the approximate value of the knight shift in tin metal. After finding the metal resonance, the true line was recorded by slowly sweeping the magnetic field in a small range about the center of the line. The frequency of the oscillator was changed in steps of about $15 \mathrm{kc} / \mathrm{s}$ and the line was plotted by slowly sweeping the magnetic field over a range of about 5 gauss around the resonant field after it was adjusted to the right value for a given frequency. The shape of the line was almost always reproducible within experimental error; however, this was an important factor in this experiment. Generally, the line did not show any distortion due to eddy current loss, and the ratio of the extreme positive value $B$ to the extreme negative value $A$ of $i$ ts derivative was about 0.7. Reference here is made to Fig. (16) where the derivative of the NMR absorption line with respect to the magnetic field is shown.

Due to the large degree of symmetry in the observed absorption curve and since the line shapes were almost unchanged within the accuracy of our measurements, no direct at tempt was made to apply any correction for the shift in the center of the line due to eddy current loss. This correction would be only a small fraction of the total line width of the observed signal.

The line widths of the observed signals as determined by the positions of the extrema of the line derivatives were measured and plotted as a function of the reciprocal magnetic field $\mathrm{H}$. 


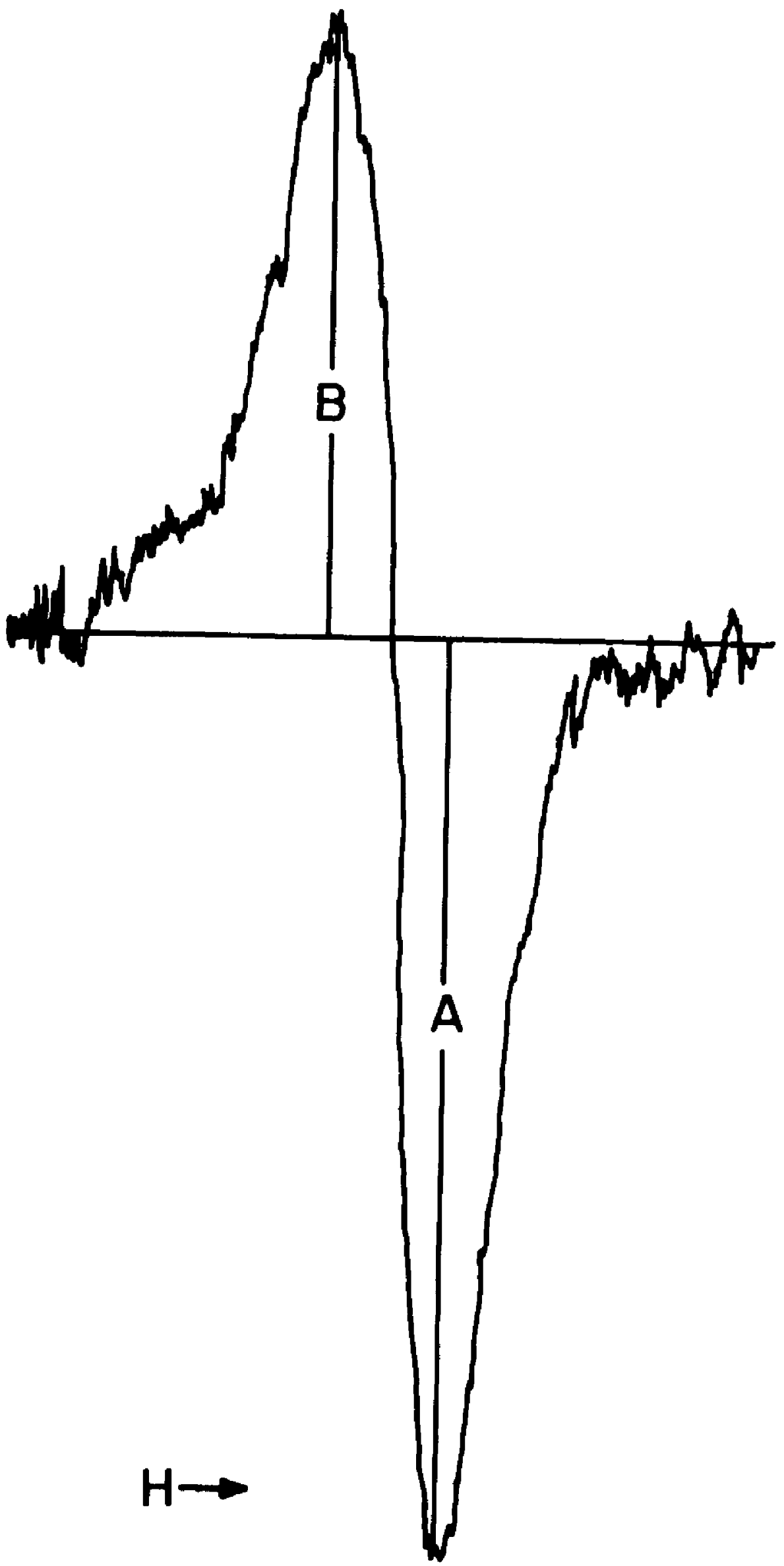

Fig. 16 
In this plot, there was an indication of weak oscillations which were not very well developed throughout the whole range of the magnetic field investigated. At places where these oscillations showed up with some clarity, the maximum estimated amplitude is about 0.3 gauss.

Though no direct correction was applied to the shift of the center of the line due to eddy current loss, it was corrected by shifting the base line to a position which would make the amplitudes of the extrema of the line derivative equal, thus bringing the apparent center closer to the true center of the absorption line. Taking into account all what has been said above, the maximum deviation of the line center from its true position would be about $20 \%$ of the line width. ${ }^{14}$ since the variation in the line width in the entire range of the magnetic field is about 0.3 gauss and since $0.3 \times 0.2=0.06$ gauss is small as compared with the experimental error (about 0.1 gauss), no significant effects in the nature and amplitude of the knight shift oscillations are produced when this correction is not taken into account.

Field calibration marks were made at intervals of one gauss while plotting the NMR lines. The frequency, which was kept constant during the sweep and which was accurate to about one part in $10^{5}$, was read directly at three different points of the sweep corresponding respectively to the approximate positions of the maximum, center, and the minimum of the line derivative. The average of these three values was used as the center frequency in 
the calculations. To obtain the field corresponding to the line center, the field calibration was plotted on the same sheet of graph paper on which the line had been traced previously. The center of the absorption line was taken midway between the positions of the maximum and minimum of its derivative. The field corresponding to the center of the line was then read directly from the linearized sweep scale.

For the purpose of comparing the magnetic field dependence of the amplitude of the Knight shift oscillations with the dependence on magnetic field of the amplitude of the magnetic susceptibility, measurements of the de Haas van-Alphen susceptibility were carried out on the same sample which was used for the Knight shift measurements. These measurements were made using the field modulation technique 16 and a signal proportional to the second derivative of the magnetization was recorded. This signal was induced in a 70 turn coil placed around the sample after being wound on a form as in the knight shift measurements. The magnetic field was applied along the [001] direction of the crystal and the axis of the pick-up coil was also in the same direction. The magnetic field was modulated at a frequency of 135 cycles per second with an amplitude of 3.5 gauss peak to peak. The signal at 270 cycles which was proportional to the second derivative of the magnetization of the sample with respect to the magnetic field was recorded using a phase-sensitive detector and Mosely $X-Y$ recorder. The signal at 135 cycles per second was a mixture of two signals: (1) The signal arising from the first derivative of the magnetization, and (2) the direct pick-up from 
the modulation field. This signal was very strong and was filtered out using a 135 cycle twin-t filter in the input circuit of the phase-sensitive detector.

Magnetization oscillations were very well developed and could be seen with full clarity starting at fields as low as 1 kilogauss. Since the de Haas van-Alphen effect is an average effect over the whole sample, it indicates that the sample was in a very good condition, the wafers were well oriented with only a small, if any, degree of misalignment.

As pointed out in Section 111 , the amplitude of an individual oscillation of period $P_{i}$ is attenuated by a factor $\left(P_{i}{ }^{2} H^{4}\right)^{-1}$ in the second derivative detection method. This can be seen as follows. Let the magnetic field be written as

$$
H(t)=H_{0}+h \sin \omega t,
$$

where $H_{0}$ is the applied d.c. magnetic field and $h \sin$ wt is the modulation field, $h$ being $i$ ts amplitude and $w$ the frequency. The magnetization may be written as

$$
M=M_{0} \sin \left(2 \pi / P_{i} H(t)\right)=M_{0} \sin f(t),
$$

where $f(t)=2 \pi / P_{i} H(t)$.

The modulation field varies the magnetization by changing the phase of the Sine term and every harmonic component of the modulation frequency has an oscillatory dependence on the inverse field for a given de Haas van-Alphen period. Writing

$$
f^{\prime}(t)=2 \pi / P_{i}\left(-H^{\prime}(t) / H^{2}(t)\right)=-2 \pi / P_{i}\left[\frac{\text { hw } \cos \omega t}{H^{2}(t)}\right],
$$




$$
\begin{gathered}
f^{\prime \prime}(t) \cong-2 \pi / P_{i} \frac{H^{\prime \prime}(t)}{H^{2}(t)}=\frac{2 \pi}{P_{i}} \frac{h \omega^{2} \sin \omega t}{H^{2}(t)}, \\
f(t)=\frac{2 \pi}{P_{i}\left(H_{0}+h \sin \omega t\right)}=\frac{2 \pi}{P_{i}}\left(1-\frac{h}{H_{0}} \sin \omega t\right),
\end{gathered}
$$

etc., we have

$$
\begin{aligned}
\frac{1}{M_{0}} \frac{\partial^{2} M}{\partial t^{2}}=\left(h \frac{2 \pi}{P_{i} H_{0}^{2}}\right) \omega^{2} \cos \frac{2 \pi}{P_{i} H_{0}} \sin \omega t-\left(h \frac{2 \pi}{P_{i} H_{0}^{2}}\right)^{2} \omega^{2} \sin \frac{2 \pi}{P_{i} H_{0}} \cos 2 \omega t \\
+\left(h \frac{2 \pi}{P_{i} H_{0}^{2}}\right)^{3} \omega^{2} \cos ^{2} \omega t \sin \omega t+\ldots
\end{aligned}
$$

where we have used the approximation

$\cos \left[\frac{2 \pi}{P_{i} H_{0}} \frac{h \sin \omega t}{H_{0}}\right] \approx 1, \quad \sin \left[\frac{2 \pi}{P_{i} H_{0}} \frac{h \sin \omega t}{H_{0}}\right]=\frac{2 \pi}{P_{i} H_{0}} \frac{h \sin \omega t}{H_{0}}$,

for small amplitude of the modulation field $h$. The signal at the second harmonic of the modulation frequency is

$$
\left[\left(h \frac{2 \pi}{P_{i} H_{0}^{2}}\right)^{2} \omega^{2} \sin \left(\frac{2 \pi}{P_{i} H_{0}}\right)\right] \cos 2 \omega t .
$$

Thus for the de Haas van-Alphen period $P_{i}$, the amplitude is attenuated by the factor $\left(P_{i}{ }^{2} H_{0}^{4}\right)^{-1}$. To obtain the correct amplitude of the magnetization, the observed amplitude value must be multiplied by $\mathrm{P}_{i}{ }^{2} \mathrm{H}_{0}{ }^{4}$ for each de Haas van-Alphen period $\mathrm{P}_{i}$, provided small amplitudes of the modulation field have been used in the detection of the de Haas van-Alphen effect. 


\section{APPENDIX II}

\section{The Fermi Surface of Tin}

The Fermi surface of white $t$ in has been studied by several investigators using different methods, e.g., de Haas van-Alphen effect, ${ }^{18}$ size effect, 35,36 galvanomagnetic effects, 37,38 magnetoacoustic effects, $39-41$ and cyclotron resonance, ${ }^{42-44}$ etc. The results have generally been interpreted in terms of the nearly free electron model presented by Gold and Priestley. ${ }^{18}$ This is sketched in Fig. (17). Most of the actual Fermi surfaces of tin are recognizable distortions of this model. The predictions of this model are as follows:

The first zone is entirely filled and the second and third ones nearly so, except for two small pockets of holes centered on the points $W$ in the second zone and a multiply connected network of cylinders, consisting of holes, in the third zone. In the fourth zone the Fermi surface is one of two sheets and consists of a singly connected surface $4(\mathrm{~b})$ formed by the intersection of a pair of electron "pancakes" along the $\Gamma$ XP(110)-type planes; this surface is surrounded by a region of unoccupied states which is in turn bounded by the multiply-connected surface $4(a)$. The fifth zone has two electron-like surfaces, the first consisting of vertical cylinders centered on $\Gamma$, and the second a large multiply-connected sheet, usually thought of as consisting of 


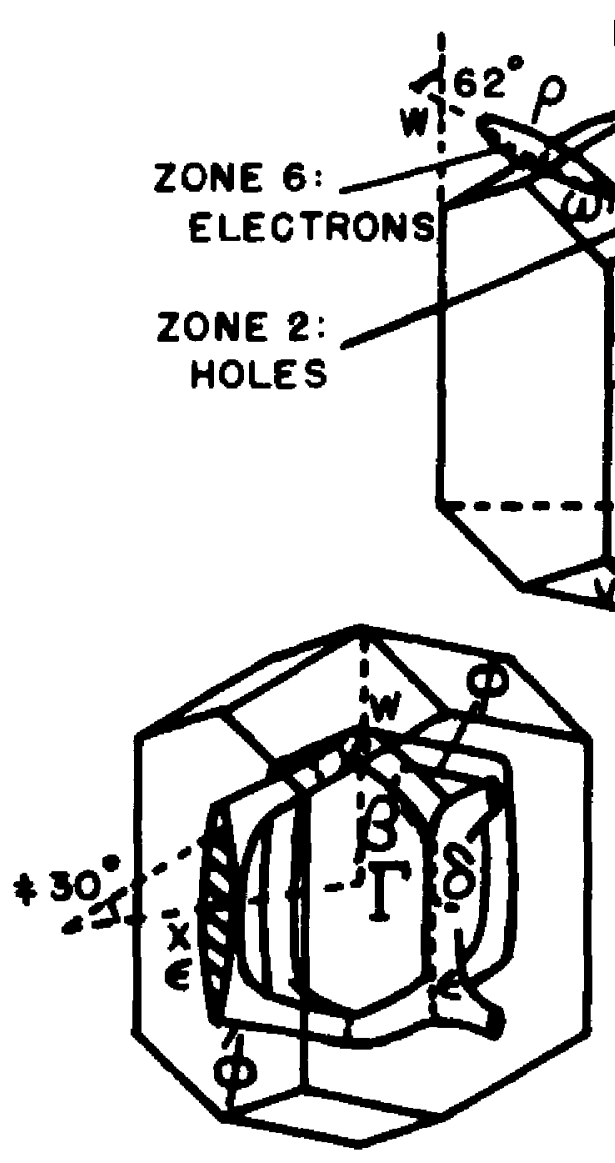

ZONE 3: HOLES

\section{[Ool]}

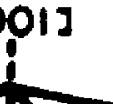

Iw 2 ZONE 6 :
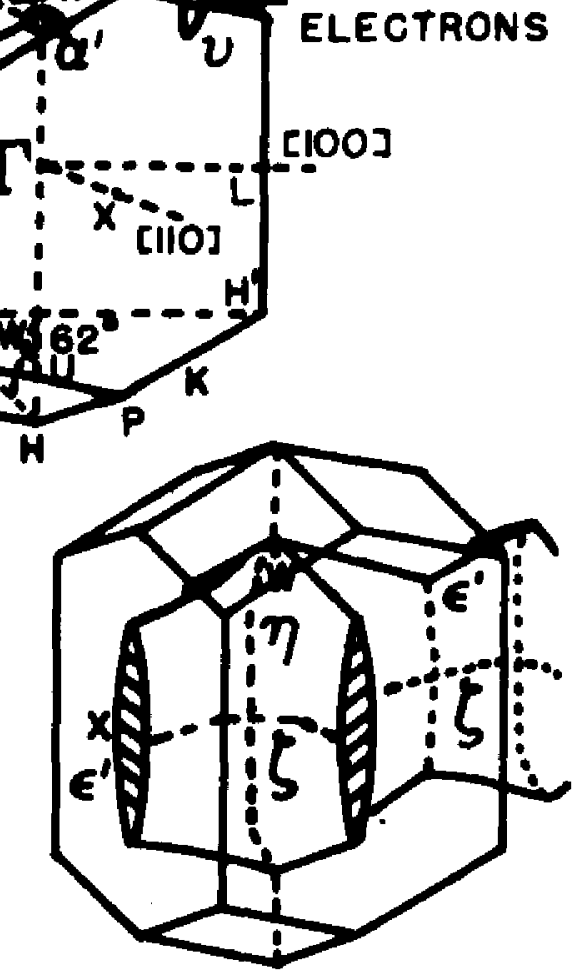

ZONE 4:(A) HOLES
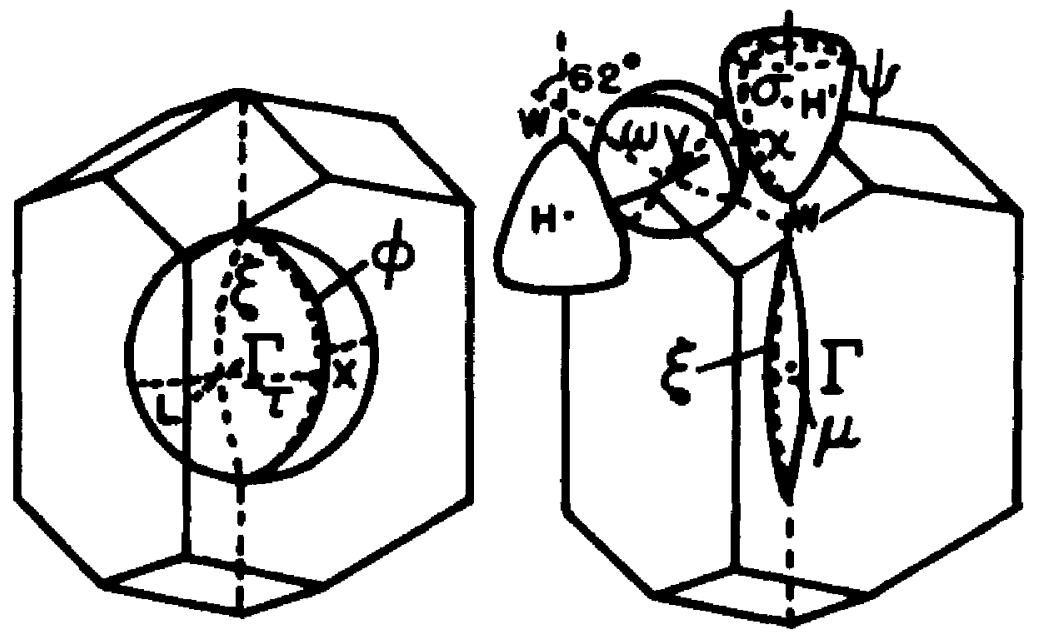

ZONE 4:(B) ELECTRONS

ZONE 5 : ELECTRONS 
"pears" and "connecting pieces". The tapered end of a "pear" near the point $W$ is called the tip, and the flat end the top. The top is roughly square, with its edges along the (100)-type directions. The "connections" are centered on the point $V$, and extend in the (100)-directions to connect a top-up pear with a top-down pear. Their cross-section is in the shape of a four-pointed star, with the distance between the points narrowing in the direction of the pear tip to which they are attached. Finally, there are the sixth zone electrons which are contained in a cigar-shaped piece around the point $V$, and in four cigar-shaped pieces centered on the points $K$, starting near the point $W$ and extending toward the corners of the pear tops.

The surface of zone $4(a)$ divides the Brillouin zone into two parts. Accordingly, every other surface should be located as a whole either in an internal or in an external part. The surfaces of zones 3 and $4(\mathrm{~b})$ lie inside the zone $4(a)$ while zones 5 and 6 lie on its outside - between the layers of the surfaces of zones 4 (a) in the repeated zone scheme.

Band structure calculations of the white tin lattice were made by M. Miasek ${ }^{24}$ using the OPW method. In these calculations the value of the parameter $v(0)$, space average of the crystal potential, was adjusted so as to cause the zone- 5 surface to exist in the neighborhood of the point $\Gamma$. In this reference it was pointed out that the hole surfaces in zone 2 should not exist. All other conclusions were in fair agreement with the free-electron model of Gold and Priestley. ${ }^{18}$ Recently G. Weisz ${ }^{19}$ 
has made band structure calculations of white tin lattice using a pseudopotential approach. According to this calculation, the crystal potential modifies the nearly free electron surfaces as follows: The zone 2 hole surface at the point $W$ disappears. In zone 3 , the hole cylinders extending from $W$ are destroyed, leaving only the cylinders along the XP lines which are nearly of the shape of a dog's bone with an extended central section, nearly cylindrical but not exactly a surface of revolution, near the point $x$. In zone 4 , electrons are introduced along $\Gamma H$ near $w$, so that there is a region, called the "neck", connecting the electrons of $4(\mathrm{~b})$ with the electrons outside $4(\mathrm{a})$. In zone 5, the cigar around $\Gamma$ disappears, and the pear-network remains essentially unchanged. In zone 6 the piece around the point $V$ disappears, while the "cigars" near the points $W$ are amalgamated into a vertical shape of square cross-section with slight prongs in the directions of the original "cigars". In these calculations, the data which is used in the determination of the fitting parameters, is taken in most part from the cyclotron sizeeffect experiments of Gantmakher. 35,36

In Fig. (18) a basal cross-section of the Fermi surface of tin is shown. 45 Magnetic breakdown connects orbit $3 \delta_{1}$ to orbit $4 a 6$ to generate the freak-orbit (i). This alteration in the orbit geometry was suggested by Gold and Priestley. 18 
$81 \cdot 6 ! 5$

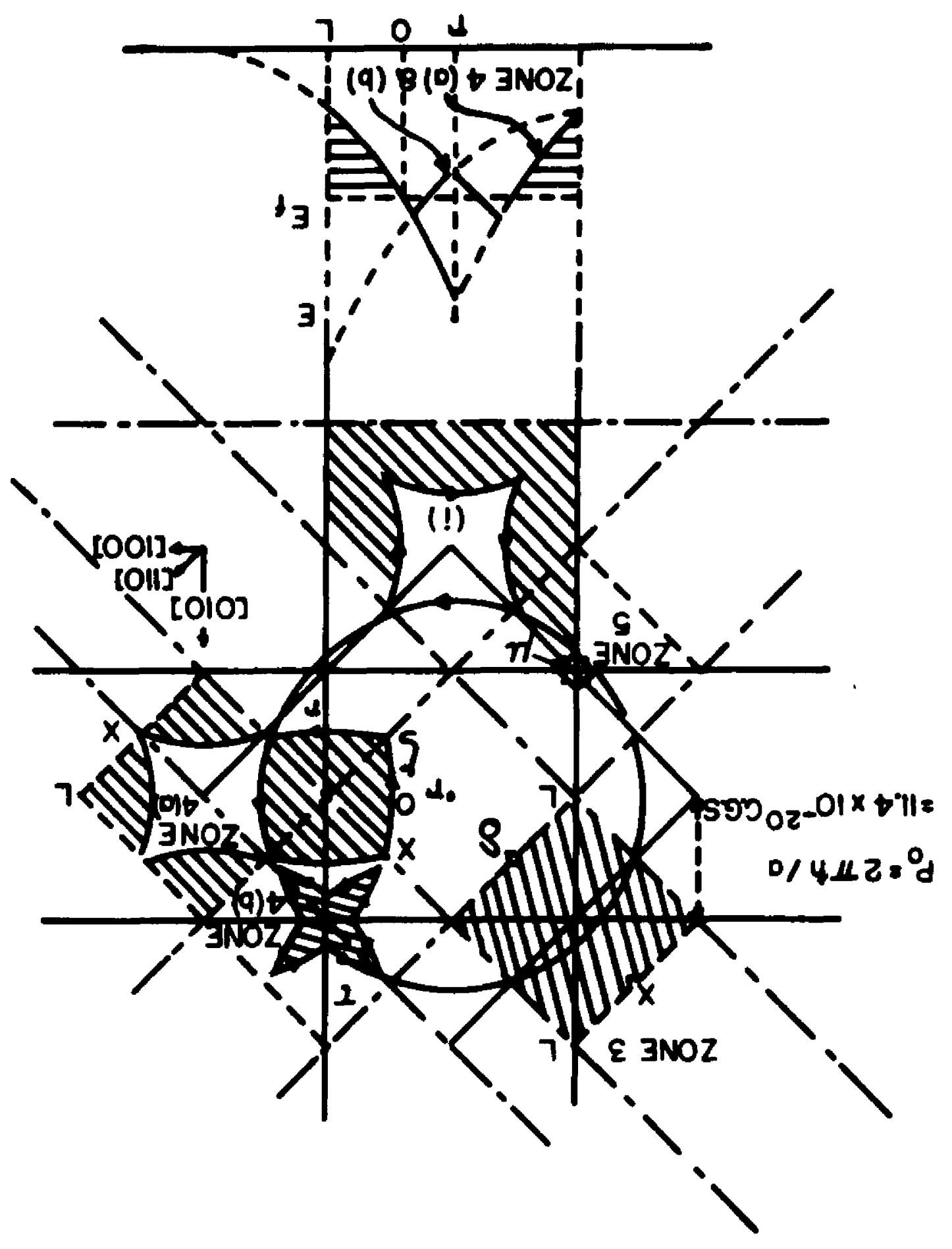


APPENDIX III

Theoretical Details

\section{A. General Considerations:}

As is known, the major contribution to the knight shift comes from the hyperfine interaction of the nuclear magnetic moment with the spins of the electrons which occupy states close to the Fermi surface. The degeneracy between the electrons of opposite spin that share the same orbital state is resolved by a magnetic field. In a metal this causes a redistribution of electrons between the two spin orientations thus generating a magnetic moment. The positive magnetic moment gives rise to the Pauli paramagnetism. This spin susceptibility is a direct measure of the density of states at the Fermi level. This paramagnetic contribution may introduce oscillatory terms in the Knight shift due to the fact that the density of electron states at the Fermi level is an oscillatory function of the inverse magnetic field. This view point has been adopted by several authors to estimate the oscillatory behavior of the Knight shift as a function of the magnetic field. Rodriguez ${ }^{5}$ in particular has emphasized the importance of these oscillations in the Knight shift measurements.

In addition to this positive paramagnetic shift, the conduction electrons may also produce a diamagnetic shift in the 
opposite direction as a consequence of their orbital motion in a magnetic field. This orbital motion produces the diamagnetic contribution to the magnetic susceptibility. Oscillatory terms may arise in the Knight shift through this contribution due to the quantization of the electron orbits in a magnetic field. Stephen ${ }^{6}$ has made a detailed calculation of this effect and has emphasized the observability of these oscillations in the Knight shift.

Glasser, ${ }^{9}$ on the other hand, takes the view point that, according to the relation (2), the behavior of the knight shift is just as sensitive to the variations in the wave function factor as it is to the variations in the magnetic susceptibility. Since most of the simple metals are nearly free-electron-like, the wave function displays a first order dependence on the lattice potential whereas the susceptibility has only a secondorder dependence. Thus in a situation where lattice effects are important, the wave function distortion may well be dominant in determining the behavior of the knight shift. Such a situation appears to be presented by the polyvalent metals. If the Fermi surface consists of small pockets of electrons or holes, its nature may be strongly affected by even moderate variations in the magnetic field, with profound consequences for the corresponding wave functions and the knight shift. In this section a review of the theories of the oscillatory Knight shift given by Stephen, ${ }^{6}$ Glasser, ${ }^{9}$ and Dolgopolov and Bystrik ${ }^{7}$ is presented. Glasser's theory seems to explain the magnitude 
of the effect quite satisfactorily. We include Stephen's theory in our discussion since this is the only calculation to date of the diamagnetic oscillatory terms which comparatively comes close to the observed effect in tin, as far as the magnitude of the Knight shift oscillations is concerned. Stephen's calculations do not bear any dependence on magnetic field of the oscillation amplitude and thus lack information in this respect. The calculations of Dolgopolov and Bystrik ${ }^{7}$ predict an amplitude of the oscillations in tin which is of about the same order of magnitude as is obtained by Stephen's ${ }^{6}$ calculations of the paramagnetic part and is far too small compared with the observed effect. These authors included only the Fermi contact part of the interaction between the conduction electrons and the nucleus in their interaction Hamiltonian. The semi-classical calculation, which they have employed, is a slight generalization of the effective-mass approximation calculation given by Stephen ${ }^{6}$; the effective-mass has been expressed in terms of the local curvature of the Fermi surface. In fact, for the special case of a parabolic band, their result for the oscillatory part of the Knight shift agrees exactly with Stephen's result for the paramagnetic oscillatory part $\sigma_{p}$.

The calculations of Rodriguez ${ }^{5}$ are not much different than Stephen's in their nature and will not be included here. This calculation predicts an amplitude of the Knight shift which is only slightly smaller in magnitude than the observed one, but it fails to meet the experimental observation for the field dependence 
of the oscillation amplitude. In the situation of magnetic breakdown, the Knight shift displays an altogether different field dependence as may be expected on the basis of the paramagnetic susceptibility.

8. Hamiltonian of the Electron-Nuclear System:

The Hamiltonian $\mathfrak{F}$ of a nuclear-electron system in the presence of a magnetic field $H$ taken along the $z$-direction can be written as 46

$$
\mathcal{K}=\mathcal{K}_{e}+\mathcal{K}_{\text {en }}+\mathcal{K}_{n}+V(\underline{r}),
$$

where

$$
\begin{aligned}
& \mathfrak{K}_{e}=\frac{1}{2 m}\left(\underline{D}-\frac{e}{c} \underline{A}\right)^{2}+2 \mu_{0} \underline{H} \cdot \underline{S} \\
& \mathcal{H}_{\mathrm{en}}=\left\{\frac{a \mu_{0}}{\hbar r^{3}}\left(\mu_{n} \cdot \underline{\underline{L}}\right)+\frac{e^{2} H \mu_{n}}{a m c^{2}}\left(\frac{x^{2}+y^{2}}{r^{3}}\right)\right\} \\
& +\left\{\frac{16 \pi}{3} \mu_{\sigma} \mu_{n} \cdot \underline{s} \delta(\underline{r})-2 \mu_{0}\left[\frac{\underline{s} \cdot \underline{\mu}_{n}}{r^{3}}-\frac{3(\underline{s} \cdot \underline{r})\left(\underline{\mu}_{n} \cdot \underline{r}\right)}{r^{5}}\right]\right\} \\
& \mathfrak{H}_{n}=\underline{n} \cdot \underline{H} \\
& \mathrm{~m} \text { = mass of an electron } \\
& \underline{\mathbf{S}}=\text { spin of an electron } \\
& \mu_{0}=\text { Bohr magneton } \\
& \mathrm{e}=\text { charge of an electron }
\end{aligned}
$$




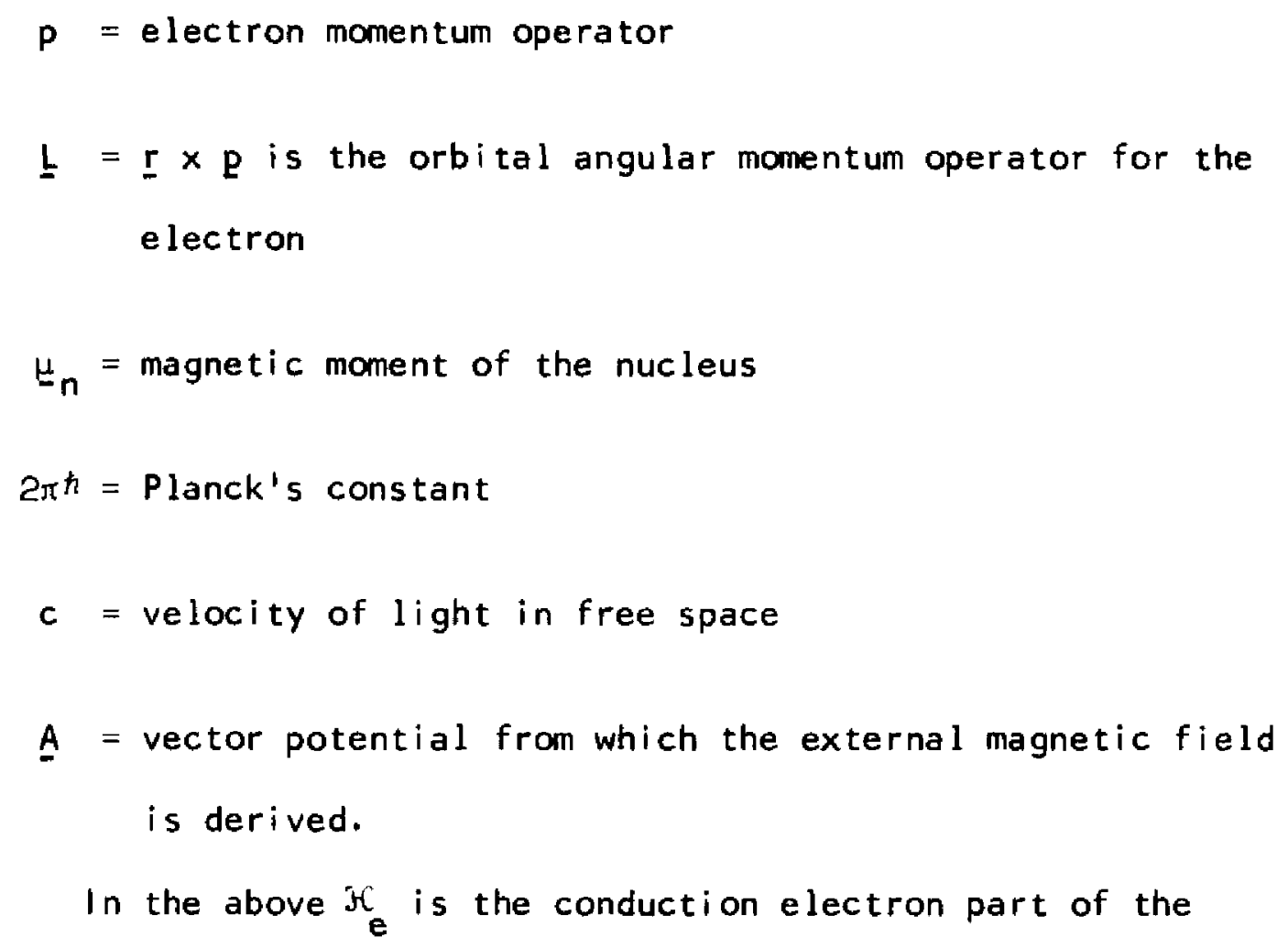
terms which can be divided in two parts:

$$
\begin{gathered}
\mathcal{H}_{\text {en }}=\mathcal{H}_{d}+\mathcal{H}_{p} \\
\mathcal{H}_{d}=\frac{2 \mu_{0}}{\hbar r^{3}}\left(\underline{\mu}_{n} \cdot \underline{L}\right)+\frac{e^{2} H \mu_{n}}{2 m c^{2}}\left(\frac{x^{2}+y^{2}}{r^{3}}\right)
\end{gathered}
$$

represents the diamagnetic part, and

$$
\xi_{p}=\frac{16 \pi}{3} \mu_{0} \underline{\mu}_{n} \cdot \underline{s} \delta(\underline{r})-2 \mu_{0}\left\{\frac{\underline{s} \cdot \mu_{n}}{r^{3}}-\frac{3(\underline{s} \cdot \underline{r})\left(\underline{\mu}_{n} \cdot \underline{r}\right)}{r^{5}}\right\}
$$

represents the paramagnetic part of the electron-nucleus inter-

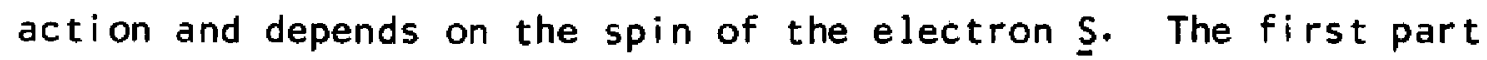
of $\mathfrak{H}_{p}$ is the Fermi contact interaction Hamiltonian and gives a major contribution to the Knight shift. The second part is 
responsible to the so-called anisotropic knight shift and vanishes for cubic crystals. $\mathcal{H}_{n}$ represents the Zeeman interaction between the nucleus and the external field and determines the basic NMR resonance frequency. $V(\underline{r})$ is the periodic lattice potential. In the present discussion, terms in $\mathfrak{H}_{\text {en }}$ represent the most important part in the Hamiltonian.

C. Stephen's Theory:

Let us consider the expression ${ }^{47}$

$$
\begin{aligned}
e^{-\gamma \mathcal{K}} \psi_{i} & =\sum_{n=0}^{\infty} \frac{(-\gamma)^{n}}{n !} \mathcal{F}^{n} \psi_{i} \\
& =\sum_{n=0}^{\infty} \frac{(-\gamma)^{n}}{n !} E_{i}^{n} \psi_{i} \\
& =e^{-\gamma E_{i}} \psi_{i}
\end{aligned}
$$

where $\mathcal{H C}^{n} \psi_{i}=E_{i}^{n} \psi_{i}$ represents operations of the Hamiltonian $\mathcal{H}$ on the wave function $\psi_{i} n$ times. Here $\psi_{i}$ 's are the normalized eigen functions of $\mathcal{H}$ with the corresponding eigen values $E_{i}$. Then

$$
\begin{aligned}
& \sum_{i} \int \psi_{i}^{*}(r) e^{-J \tau \gamma} \psi_{i}(\underline{r}) d \tau \\
& =\Sigma_{i} e^{-\gamma E_{i} \int \psi_{i}^{*} \psi_{i} d \tau} \\
& =\Sigma_{i} e^{-\gamma E_{i}}=Z(\gamma)
\end{aligned}
$$


where $Z(\gamma)$ is the partition function of the system. Here $Y=\frac{1}{k T}$ where $k$ is Boltzmann constant and $T$ is the absolute temperature and $d \tau=d^{3} \underline{r}$ is the element of volume. We have

$$
\begin{aligned}
Z(\gamma) & =\int \sum_{i} \psi_{i}^{*}(\underline{r}) e^{-\gamma \mathcal{K}_{k}} \psi_{i}(\underline{r}) d \tau \\
& =\int \Psi(\underline{r}, \underline{r}, \gamma) d \tau
\end{aligned}
$$

where we define the density matrix $\Psi\left(\underline{r}^{\prime}, \underline{r}, \gamma\right)$ as follows:

$$
\Psi\left(\underline{r}^{\prime}, \underline{r}, \gamma\right)=\Sigma_{i} \psi_{i}^{*}\left(\underline{r}^{\prime}\right) e^{-\gamma^{3} K_{\psi}} \psi_{i}(\underline{r})
$$

Differentiating with respect to $\gamma$, gives

$$
\begin{aligned}
-\frac{\partial \Psi}{\partial \gamma} & =\Sigma_{i} \psi_{i}^{*}\left(\underline{r}^{\prime}\right) \mathcal{H e}^{-\gamma \mathcal{H}} \psi_{i}(\underline{r}) \\
& =\mathcal{H} \Sigma_{i} \psi_{i}^{*}\left(\underline{r}^{\prime}\right) \mathrm{e}^{-\gamma \mathcal{H} \psi_{i}(\underline{r})} \\
& =\mathcal{H} \Psi\left(\underline{r}^{\prime}, r, \gamma\right)
\end{aligned}
$$

Since $\mathcal{K}$ operates only on $\psi_{i}(\underline{r})$. Equation (5) represents the differential equation satisfied by $\Psi$. We also have

$$
\Psi\left(\underline{r}^{\prime}, \underline{r}, 0\right)=\Sigma_{i} \psi_{i}^{*}\left(\underline{r}^{\prime}\right) \Psi(\underline{r})=\delta\left(\underline{r}-\underline{r}^{\prime}\right)
$$

which is the condition of completeness of the system of wave functions $\psi_{i}$.

To calculate the oscillatory terms, Stephen ${ }^{6}$ included both the diamagnetic and paramagnetic terms in the interaction Hamiltonian 
with $m$ replaced by $m^{*}$, the effective mass of the electron. Thus

$$
\begin{array}{r}
\mathcal{H}_{d}(\underline{r})=-2 i \mu_{0}^{*} \mu_{n} \frac{1}{r^{3}}\left(x \frac{\partial}{\partial y}-y \frac{\partial}{\partial x}\right)+\frac{e^{2} H \mu_{n}}{\partial m^{*} c^{2}}\left(\frac{x^{2}+y^{2}}{r^{3}}\right) \\
\mathcal{H}_{p}(\underline{r})=\mathcal{F}_{p}(\underline{r}) S_{z}=\frac{16 \pi}{3} \mu_{0} \mu_{n} \delta(\underline{r}) S_{z}+2 \mu_{0} \mu_{n}\left(\frac{\left(z^{2}-r^{2}\right.}{r^{5}}\right) S_{z}
\end{array}
$$

where $x, y, z$ are rectangular components of $\underline{r}$.

The density matrix, or propagator, can be written in a generalized form as

$$
\Psi\left(\underline{r}_{1}, \underline{r}_{2}, \gamma_{1}-\gamma_{2}\right)=\theta\left(\gamma_{1}-\gamma_{2}\right) \Sigma_{i} \psi_{i}^{*}\left(\underline{r}_{2}\right) e^{-\left(\gamma_{1}-\gamma_{2}\right) H} \psi_{i}\left(r_{1}\right)
$$

where

$$
\theta(x)=\left\{\begin{array}{l}
0 \text { for } x<0 \\
1 \text { for } x>1
\end{array}\right.
$$

is the step function. The propagator Eq. (9) satisfies the equation

$$
\left(\frac{\partial}{\partial \gamma_{1}}+\mathcal{H}\left(\underline{r}_{1}\right)\right) \Psi\left(\underline{r}_{1}, \underline{r}_{2}, \gamma_{1}-\gamma_{2}\right)=\delta\left(\underline{r}_{1}-\underline{r}_{2}\right) \delta\left(\gamma_{1}-\gamma_{2}\right) .
$$

If $\mathcal{H}$ is replaced by $\mathcal{K}_{0}=\mathcal{H}_{\mathrm{e}}$ in Eq. (10), the solution is the Green's function $G\left(r_{1}, r_{2}, \gamma_{1}-\gamma_{2}\right)$ which is given by

$$
G\left(\underline{r}_{1}, \underline{r}_{2}, \gamma_{1}-\gamma_{2}\right)=\theta\left(\gamma_{1}-\gamma_{2}\right) \Sigma_{i} \psi_{i}^{*}\left(\underline{r}_{2}\right) e^{-\left(\gamma_{1}-\gamma_{2}\right) \mathcal{H}_{0}} \psi_{i}\left(\underline{r}_{1}\right)
$$

and satisfies the appropriate boundary conditions. In terms of 
the Green's function, the solution of Eq. (10) is

$$
\begin{gathered}
\Psi\left(\underline{r}_{1}, \underline{r}_{2}, \gamma_{1}-\gamma_{2}\right)=G\left(\underline{r}_{1}, \underline{r}_{2}, \gamma_{1}-\gamma_{2}\right) \\
-\int d \underline{r}^{\prime} d \gamma^{\prime} G\left(\underline{r}_{1}, \underline{r}^{\prime}, \gamma_{1}-\gamma^{\prime}\right) \mathcal{F}^{\prime}\left(\underline{r}^{\prime}\right) \Psi\left(\underline{r}^{\prime}, \underline{r}_{2}, \gamma^{\prime}-\gamma_{2}\right)
\end{gathered}
$$

where $\mathcal{H}^{\prime} \equiv \mathcal{K}$ en

In the present discussion, we only require the part of the partition function which is linear in the applied perturbation. To obtain this we replace $\Psi$ by $G$ under the integral sign in Eq. and write

$$
\begin{gathered}
Z^{\prime}(\gamma)=\int \Psi(\underline{r}, \underline{r}, \gamma) d \underline{r} \\
=-\int d \underline{r} \int d \underline{r}^{\prime} d \gamma^{\prime} G\left(\underline{r}, \underline{r}^{\prime}, \gamma-\gamma^{\prime}\right) \mathcal{H}^{\prime}\left(\underline{r}^{\prime}\right) G\left(\underline{r}^{\prime}, \underline{r}, \gamma\right)
\end{gathered}
$$

If we integrate over the spin variables, then Eq. (9) can be written as, for $\gamma>0$,

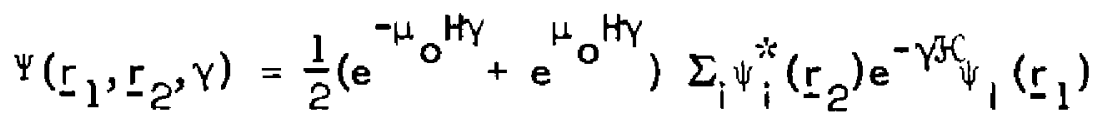

$$
\begin{aligned}
& =\frac{1}{2} e^{-\mu_{0} H \gamma} \Sigma \psi_{i}^{*}\left(\underline{r}_{2}\right) e^{-\gamma\left[\mathcal{H}_{0}+\frac{1}{2} \mathcal{F}_{p}\left(\underline{r}_{1}\right)+\mathcal{K}_{d}\left(\underline{r}_{1}\right)\right]} \psi_{i}\left(\underline{r}_{1}\right) \\
& +\frac{1}{2} e^{\mu \mu_{0}^{H \psi}} \Sigma_{i} \psi_{i}^{*}\left(\underline{r}_{2}\right) e^{-\gamma\left[\mathcal{H}_{0}-\frac{1}{2} \mathcal{K}_{p}\left(\underline{r}_{1}\right)+\mathcal{H}_{d}\left(\underline{r}_{1}\right)\right]} \psi_{i}\left(\underline{r}_{1}\right) \\
& \Psi(\underline{r}, \underline{r}, \gamma)=\frac{1}{2}\left(e^{-\mu_{0} H \gamma}+e^{\mu_{0}^{H}+\gamma}\right) \sum_{i} \psi_{i}^{*}(\underline{r}) e^{-\gamma\left[\mathcal{F}_{0}+\mathcal{K}_{d}(\underline{r})\right]} \psi_{i}(\underline{r})
\end{aligned}
$$


66

$$
\begin{aligned}
& +\frac{1}{2} e^{-\mu_{0}^{H Y \gamma}} \Sigma_{i} \psi_{i}^{*}(\underline{r}) e^{-\gamma\left[\mathcal{F}_{0}+\frac{1}{2} \mathcal{F}_{p}\right]} \psi_{\psi_{i}}(r) \\
& +\frac{1}{2} e^{\mu \mu_{0}^{H Y}} \Sigma_{i} \psi_{i}^{*}(r) e^{-\gamma\left[\mathcal{H}_{0}-\frac{1}{2} \mathcal{F}_{p}\right]} \psi_{\psi_{i}}(\underline{r}) .
\end{aligned}
$$

In view of Eq. (13) this leads to a separation of the partition function into the diamagnetic and paramagnetic parts:

$$
\begin{aligned}
& z_{d}(\gamma)=-\frac{1}{2}\left(e^{-\mu_{0} H \gamma}+e^{\mu_{0} H \gamma}\right) \int d \gamma^{\prime} \int d \underline{r} d \underline{r}^{\prime} \\
& G\left(\underline{r}, \underline{r}^{\prime}, \gamma-\gamma^{\prime}\right) \mathcal{H}_{d}\left(\underline{r}^{\prime}\right) G\left(\underline{r} \underline{r}^{\prime}, \underline{r}, \gamma^{\prime}\right) \\
& z_{p}(\gamma)=-\frac{1}{2}\left(e^{-\mu_{0} H \gamma}-e^{\mu_{0} H \gamma}\right) \int d \gamma^{\prime} \int d \underline{d r} \underline{r}^{\prime} \\
& G\left(\underline{r}, \underline{r}^{\prime}, \gamma-\gamma^{\prime}\right) \frac{1}{2} H_{P}\left(\underline{r}^{\prime}\right) G\left(\underline{r}^{\prime}, \underline{r}, \gamma^{\prime}\right) .
\end{aligned}
$$

Putting $\gamma^{\prime}=\gamma s$, we obtain

$$
\begin{aligned}
& Z_{d}(\gamma)=-\gamma \cosh \left(\mu_{0}+\gamma \gamma\right) \int_{0}^{1} d s \int d \underline{r} d \underline{r}^{\prime} \\
& G\left(\underline{r}, \underline{r}^{\prime}, \gamma(1-s)\right) K_{d}\left(\underline{r}^{\prime}\right) G\left(\underline{r}^{\prime}, \underline{r}, \gamma s\right) \\
& Z_{p}(\gamma)=\frac{1}{2} \gamma \sinh \left(\mu_{0}+r y\right) \int_{0}^{1} d s \int d \underline{r} d \underline{r}^{\prime} \\
& G\left(\underline{r}, \underline{r}^{\prime}, \gamma(1-s)\right) K_{p}\left(\underline{r}^{\prime}\right) G\left(\underline{r}^{\prime}, \underline{r}, \gamma s\right)
\end{aligned}
$$


The Green's function in Eqs. (15) and (16) is the density matrix for a spinless electron in a uniform magnetic field which has been evaluated by Sondheimer and Wilson ${ }^{47}$ and is given by

$$
\begin{gathered}
G\left(\underline{r}, \underline{r}^{\prime}, \gamma\right)=\left(\frac{m^{*}}{2 \pi \hbar^{2}}\right)^{3 / 2} \frac{\mu_{0}^{*} H y}{\sinh \left(\mu_{0}^{*} H+\gamma\right)} \\
x \exp \left[-\frac{m^{*}}{2^{\hbar^{2}} \gamma}\left[2 i \mu_{0}^{*} H y\left(x y^{\prime}-y x^{\prime}\right)\right.\right. \\
\left.+\mu_{0}^{*} H \gamma \operatorname{coth}\left(\mu_{0}^{*} H \gamma\right)\left\lfloor\left(x-x^{\prime}\right)^{2}+\left(y-y^{\prime}\right)^{2}+\left(z-z^{\prime}\right)^{2}\right\}\right] .
\end{gathered}
$$

Substituting Eqs. (8) and (17) in Eq. (16) we find that the second term of Eq. (8) vanishes identically and the remaining term yields on integration the result

$$
z_{p}(\gamma)=\frac{8 \pi}{3} \mu_{0} \mu_{n}\left(\frac{m^{*}}{2 \pi n^{2}}\right)^{3 / 2} \mu_{0}^{* k_{y}} l / 2 \frac{\sinh \left(\mu_{0}+\gamma\right)}{\sinh \left(\mu_{0}^{*}+\gamma\right)} .
$$

Again, substitution of Eqs. (7) and (17) into Eq. (15) leads to an integral of the form

$$
\begin{aligned}
& \int d \underline{r} \underline{d r^{\prime}}\left(\frac{{\underline{x^{\prime}}}^{2}+y^{\prime 2}-x x^{\prime}-y y^{\prime}}{r^{\prime}}\right) \exp \left[-\alpha^{2}\left\{\left(x-x^{\prime}\right)^{2}+\left(y-y^{\prime}\right)^{2}\right\}\right] \\
& x \exp \left[-\delta^{2}\left(z-z^{1}\right)^{2}\right],
\end{aligned}
$$

where $\delta^{2}=m^{*} / 2 \hbar^{2} \gamma s(1-s)$,

$$
\alpha^{2}=\frac{m_{\mu}^{*} \mu_{0}^{*} H}{2 \hbar^{2}} \frac{\sinh \left(\mu_{0}^{*} H Y\right)}{\sinh \left(\mu_{0}^{*} H y s\right) \sinh \left[\mu_{0}^{*} H Y(1-s)\right]} .
$$


Here we have used the approximations $\sinh \mu_{0}^{*}{ }_{0} H Y s=\mu_{0}^{*}{ }_{0}^{*} H Y$, $\cosh \mu_{0}^{*}$ Hrs $\approx 1$, etc.

It is convenient to integrate Eq. (19) over the volume contained within the ellipsoid $\alpha^{2} x^{2}+\alpha^{2} y^{2}+\delta^{2} z^{2}=R^{2}$, although the final result is independent of the shape of the system. The scale transformation $\alpha_{x} \rightarrow x, \alpha_{y} \rightarrow y, \delta z \rightarrow z$ turns this ellipsoid into a sphere and brings Eq. (19) into the form

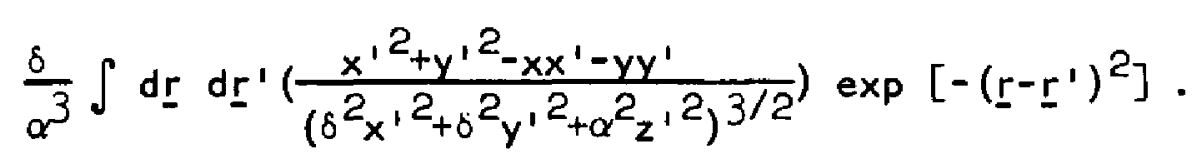

Keeping $\underline{r}^{\prime}$ fixed, we integrate over all angles of $\underline{r}$ with respect to $\underline{r}^{\prime}$ by choosing the axis of $\underline{I}$ along $\underline{I}^{\prime}$. This gives

$$
\begin{gathered}
\frac{4 \pi^{2} \delta}{\alpha^{3}} \int_{0}^{\pi} d \theta^{\prime} \frac{\sin ^{3} \theta^{\prime}}{\left(\delta^{2} \sin ^{2} \theta^{\prime}+\alpha^{2} \cos ^{2} \theta^{\prime}\right)^{3 / 2}} \int_{0}^{R} r^{2} d r \int_{0}^{R} r^{2} d r^{\prime} \\
x \exp \left(-r^{2}-r^{\prime 2}\right)\left\{\frac{\sinh \left(2 r r^{\prime}\right)}{r r^{\prime 2}}+\frac{\sinh \left(2 r r^{\prime}\right)}{2 r r^{\prime 4}}-\frac{\cosh \left(2 r r^{\prime}\right)}{r^{\prime 3}}\right\} .
\end{gathered}
$$

The first integral gives $\left(\alpha^{2}>\delta^{2}\right)$

$$
\frac{2 \alpha}{\delta^{2}\left(\alpha^{2}-\delta^{2}\right)}-\frac{2}{\left(\alpha^{2}-\delta^{2}\right)^{3 / 2}} \ln \left(\frac{\alpha^{2}+\left(\alpha^{2}-\delta^{2}\right)^{1 / 2}}{\delta}\right) .
$$

The radial integral may be simplified by integrating the last term by parts (on integrating cosh $2 r^{\prime}$ ). This cancels the first two 
69

terms and gives

$$
\int_{0}^{R} r^{2} d r \exp \left(-r^{2}\right)\left(1-\exp \left(-R^{2}\right) \frac{\sinh 2 r R}{2 r R}\right) \text {. }
$$

In the limit of $R \rightarrow \infty$, this gives $\frac{1}{8} \pi^{1 / 2}$. Collecting, we obtain finally

$$
\begin{aligned}
& z_{d}(\gamma)=-8 \pi \mu_{0}^{*} \mu_{n}\left(\frac{m^{*}}{2 \pi \pi^{2}}\right)^{3 / 2}\left(\mu_{0}^{*} H\right)^{2} \frac{\gamma^{3 / 2} \cosh \left(\mu_{0} H Y\right)}{\sinh \left(\mu_{0}^{*} H Y\right)} \\
& x \int_{0}^{1} d s s(1-s)\left\{(A-1)^{-1}-A^{-1 / 2}(A-1)^{-3 / 2} \ln \left[A{ }^{1 / 2}+(A-1)^{1 / 2}\right]\right\}
\end{aligned}
$$

where

$$
A=\frac{\mu_{0}^{*} \operatorname{Hrs}(1-s) \sinh \left(\mu{ }_{0}^{*} H \gamma\right)}{\sinh \left(\mu_{0}^{*} H y s\right) \sinh \left[\mu_{0}^{*} H y(1-s)\right]} .
$$

To obtain the Knight shift we must find the free energy $F$, since

$$
\sigma=-\frac{1}{H}\left(\frac{\partial F}{\partial \mu_{n}}\right) \mu_{n}=0
$$

We define a function $z(E)$ as the inverse Laplace transformation of the classical partition function

$$
z(E)=\frac{1}{2 \pi i} \int_{c-i \infty}^{c+i \infty} d \gamma e^{E \gamma} \frac{z(\gamma)}{\gamma^{2}}
$$


where $c$ is a positive constant chosen so that all the singularities of $Z(\gamma)$ lie to the left of the line $\left(c-i \infty, c+i^{\infty}\right)$. In terms of the function $z(E)$, the free energy is given by

$$
F=2 \int_{0}^{\infty} d E z(E) \frac{\partial f_{0}}{\partial E}
$$

where $f_{0}=[1+\exp Y(E-\zeta)]^{-1}$ is the Fermi function.

For the paramagnetic terms, when we substitute $Z_{p}(\gamma)$ for $Z(\gamma)$ in Eq. (24), the integrand is found to have a series of simple poles along the imaginary axis with a branch point at $\gamma=0$. The integral is evaluated by the method of residues around a closed contour in the form of a large semi-circle covering the left portion of the complex $\gamma$-plane with a cut along the negative real axis such that the whole contour does not pass through any of the poles of the integrand. Sandheimer and Wilson ${ }^{47}$ evaluated such an integral for the magnetic susceptibility which follows exactly the same behavior. The result for the oscillatory part of the paramagnetic Knight shift is

$$
\sigma_{p}=\frac{4 \pi^{2} N \mu_{0}^{2}}{\gamma V \zeta_{0}}\left(\frac{1}{\mu_{0} H \zeta_{0}} \frac{m}{m^{*}}\right) 1 / 2 \sum_{n=1}^{\infty} \frac{(-1)^{n} \sin \left(n \pi \frac{m^{*}}{m}\right) \cos \left(\frac{n \pi \sigma_{0}}{\mu_{0}^{*} H}-\frac{\pi}{4}\right)}{n^{1 / 2} \sinh \left(\frac{n \pi^{2}}{\mu_{0}^{*} H \gamma}\right)}
$$

where

$$
S_{0}=\left(9 \pi \frac{N^{2}}{v^{2}}\right)^{1 / 3}\left(\frac{\pi \hbar^{2}}{2 m^{\frac{1}{k}}}\right) .
$$

These oscillatory terms arise from the poles of $z_{p}(\gamma)$ and 
are a reflection of the fact that the density of states is an oscillatory function of $\frac{l}{H}$. The amplitude of the first oscillation is the most dominant of all and increases as $H^{1 / 2}$.

The only significant contribution to the diamagnetic part of the oscillatory knight shift comes from the second part of $z_{d}(\gamma)$. Hence for the purpose of calculations, we can approximately write

$$
\begin{aligned}
& z_{d}(\gamma)=8 \pi \mu_{0}^{*} \mu_{n}\left(\frac{m^{*}}{2 \pi \pi^{2}}\right)^{3 / 2}\left(\mu_{0}^{*} H\right)^{2} \frac{\gamma^{3 / 2} \cosh \left(\mu_{0} H \gamma\right)}{\sinh \left(\mu_{0}^{*} H Y\right)} \\
& \times \int_{0}^{1} d s \quad s(1-s) A^{-1 / 2}(A-1)^{-3 / 2} \ln \left[A^{1 / 2}+(A-1)^{1 / 2}\right] .
\end{aligned}
$$

This integral has a series of branch points along the imaginary axis at $\mu_{0}^{*} H \gamma=n \pi i, n=0, \pm 1, \ldots$ in the complex $\gamma$-plane. To make the integral single valued we make a series of cuts in the plane from each point $n \pi i$ to $-\infty$ parallel to the real axis [see Fig. (19)]. The contour is completed properly so that the integrand does not have any poles or singularities within it and we need only consider the integrals along the loops encircling the branch cuts. Denoting the loop encircling the branch point $\mu_{0}^{*} H Y=n \pi i$ by $\sigma_{n}$, e.g., ABCDE in Fig. (19), we have from Eq. (24)

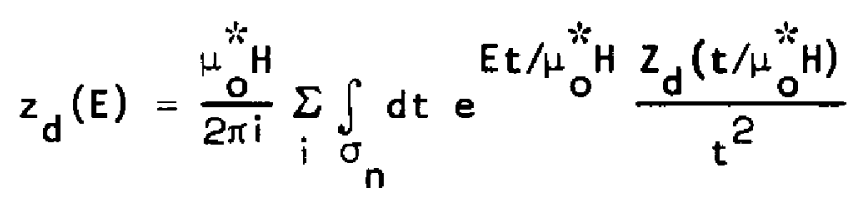




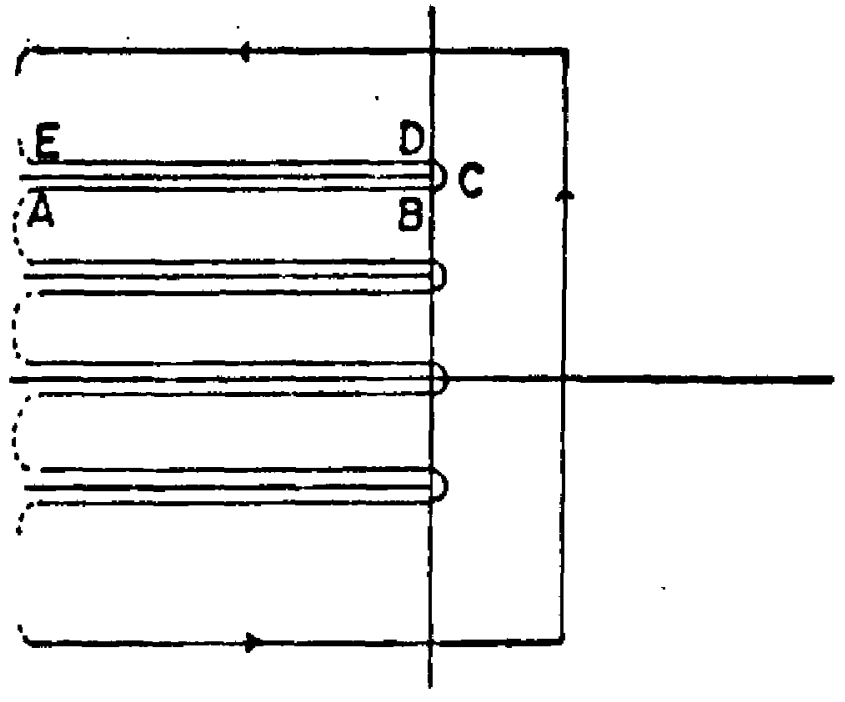

Fig. 19 
where $t=\mu_{0}^{*} H y$. To obtain the most important oscillatory term it will be sufficient to know the value of $z_{d}$ at $t=n \pi i$. For simplicity we define

$J(t)=[\sinh t]^{1 / 2} \int_{0}^{1} d s s(1-s) A^{-1 / 2}(A-1)^{-3 / 2} \ln \left[A^{1 / 2}+(A-1)^{1 / 2}\right]$

For $t=n \pi i$ and $0 \leq s \leq l$ we can write

$$
\sinh \operatorname{ts} \sinh t(1-s)=|\sin n \pi s|^{2} e^{i n \pi}
$$

and obtain

$$
\begin{gathered}
J(t)=-\frac{\pi}{2} e^{1 / 2 n \pi i} \int_{0}^{l} d s[s(1-s)]^{1 / 2}|\sin n \pi s| \\
J(t)=-\frac{\pi}{2} e^{1 / 2 n \pi i} I(n)
\end{gathered}
$$

where $I(n)$ may be evaluated numerically. 20

Let us now consider the integral around $\sigma_{n}(n \neq 0)$ in Eq.

and put $t=n \pi i+x$. This integral becomes (omitting constants)

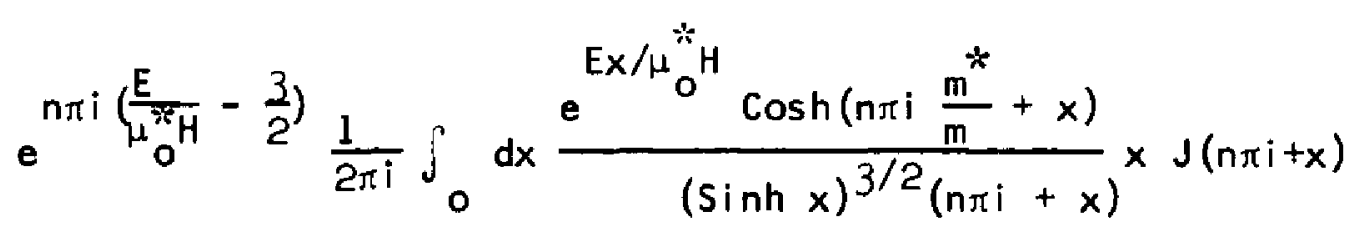

for $\sinh (n \pi i+x)=e^{n \pi i} \sinh x$. Since $x$ is small, the above expression is approximately

$$
\approx e^{n \pi i\left(\frac{E}{\mu_{0}^{*} H}-1\right)} \frac{\cos \left(n \pi \frac{m^{*}}{m}\right)}{2 n i} \frac{1(n)}{2 \pi i} j_{\sigma} d x \frac{e \frac{E x}{\mu_{0}^{*} H}}{x^{3 / 2}}
$$


Let $y=\frac{E X}{H_{0}^{*} H}$, then

$$
\begin{aligned}
& \frac{1}{2 \pi i} \int_{\sigma} d x \frac{e^{E x / \mu_{0}^{*} H}}{x^{3 / 2}}=\left(\frac{E}{\mu_{0}^{*} H}\right)^{1 / 2} \frac{1}{2 \pi i} \int_{\sigma} e^{y} y^{-3 / 2} d y \\
&=\left(\frac{E}{H_{0}^{*} H}\right)^{1 / 2} \frac{1}{\Gamma\left(\frac{3}{2}\right)}=\frac{2}{\sqrt{\pi}}\left(\frac{E}{H_{0}^{*} H}\right)^{1 / 2}
\end{aligned}
$$

The above equation is, therefore

$$
=\left(\frac{E}{\mu_{0}^{*} H}\right)^{1 / 2} \frac{(-1)^{n} I(n)}{n i \pi} \cos \left(\frac{n \pi m^{*}}{m}\right) e^{n \pi i E / \mu_{0}^{*} H} .
$$

When this is combined with the corresponding term for $-n$, we obtain the contribution to $z_{d}(E)$ from all those branch points with $n \neq 0$

$$
\begin{gathered}
z_{d}(E)=-\left(\frac{m^{*}}{2 \pi \hbar^{2}}\right)^{3 / 2} 16 \pi^{1 / 2} \mu_{0}^{* 2} \mu_{n} H E{ }^{1 / 2} \sum_{n=1}^{\infty} \frac{(-1)^{n} I(n)}{n} \\
\times \cos \left(\frac{n \pi m^{*}}{m}\right) \sin \left(\frac{n \pi E}{\mu_{0}^{*} H}\right) .
\end{gathered}
$$

The free energy is, therefore

$$
F=2 \int_{0}^{\infty} d E z_{d}(E) \frac{\partial f^{0}}{\partial E}
$$

where

$$
f^{0}=\left[1+e^{\gamma(1-\zeta)}\right]^{-1} .
$$




$$
\begin{gathered}
F=-2\left(\frac{m^{*}}{2 \pi \hbar^{2}}\right)^{3 / 2} 16 \pi^{1 / 2} \mu_{0}^{*} \mu_{N} H \sum_{n=1}^{\infty} \frac{(-1)^{n} 1(n)}{n} \cos \left(\frac{n \pi m^{*}}{m}\right) \\
\quad \times Y \int_{0}^{\infty} d E E^{1 / 2} \sin \left(\frac{n \pi E}{\mu_{0}^{*} H}\right) \frac{e^{\gamma(E-\zeta)}}{\left[1+e^{\gamma(E-\zeta)}\right]^{2}} \cdot
\end{gathered}
$$

Thus we need to evaluate the integral

$$
Y \operatorname{Im} \int_{0}^{\infty} d E E^{l / 2} e^{i n \pi E / H_{0}^{*} H} \frac{e^{\gamma(E-\zeta)}}{\left[1+e^{\gamma(E-\zeta)}\right]^{2}} \cdot
$$

This has been done by Glasser. ${ }^{20}$ The result, neglecting terms of order $(Y \zeta)^{-1}$, is

$$
\frac{\pi^{2} n \zeta^{1 / 2}}{\mu_{0}^{*} H \gamma} \frac{\sin \left(\frac{n \pi \zeta}{\mu_{0}^{*} H}\right)}{H_{0}^{*} H y \sinh \left(\frac{n \pi \pi^{2}}{\mu_{0}^{*} H}\right)} \text {. }
$$

Since $\sigma=-\frac{1}{H}\left(\frac{\partial F}{\partial \mu_{N}}\right)_{\mu}$ we finally obtain for the diamagnetic oscillatory Knight shift the expression:

$$
\sigma_{d}(05 c)=\frac{6 N \pi^{3} \mu_{0}^{*}}{V \zeta_{0} Y H} \sum_{n=1}^{\infty} \frac{(-1)^{n} I(n) \cos \left(\frac{n \pi m^{*}}{m}\right) \sin \left(\frac{n \pi \sigma_{0}}{\mu_{0}^{*} H}\right)}{\sinh \left(\frac{n \pi^{2}}{\mu_{0}^{*} H H}\right)}
$$

which differs from Stephen's result by a factor of $(-2)$. 
The amplitudes of the first oscillations would be the most dominant amongst all the oscillatory terms. In the approximation $\frac{\pi^{2} k T}{{ }^{*} * H}<1$ which can be obtained at low temperatures in the liquid helium range and at moderately high magnetic fields of the order of $10^{4}$ gauss, the amplitudes of the first oscillations are, from Eqs. (26) and (36)

$$
\begin{aligned}
& \left(\sigma_{p}\right)_{1}=\frac{4 N}{V}\left(\frac{\mu_{0}}{\sigma_{0}}\right)^{3 / 2}\left(\frac{m}{m}\right)^{1 / 2} \mu_{o}^{*} H^{1 / 2} \\
& \left(\sigma_{d}\right)_{1}=\frac{6 N \pi \mu_{0}^{*^{2}}}{V \sigma_{0}} 1(1), 1(1) \sim 0.3 .
\end{aligned}
$$

For $\operatorname{tin}$ where $\zeta_{0}=1.58 \times 10^{-11}$ ergs ${ }^{19}, \mathrm{~m}^{*} \sim 0.1 \mathrm{~m}_{0}$ we have for $\mathrm{H} \sim 10^{4}$ gauss

$$
\left(\sigma_{p}\right)_{1} \sim 10^{-3}\left(\sigma_{d}\right)_{1}
$$

and hence only $\left(\sigma_{d}\right)_{1}$ need be considered. Putting $\frac{N}{V}=\frac{\sigma_{0}^{3 / 2}}{3 \pi^{2}{ }^{3}}\left(2 m^{*}\right)^{3 / 2}$ we obtain

$$
\left(\sigma_{d}\right)_{1} \approx 4 \frac{2^{1 / 2}}{\pi \hbar^{3}} \mu_{0}^{* 2} \sigma_{0}^{1 / 2} m^{* 3 / 2} I(1)
$$

which is independent of both the magnetic field and the temperature. A numerical estimate shows that we can have at best $\left(\sigma_{d}\right)_{1} \sim 6.8 \times 10^{-6}$, on taking into account the demagnetizing factor $\left(d \approx \frac{1}{2}\right)$. This is about one-seventh of the observed amplitude. 
0. Glasser's Theory:

In the nearly free electron approximation, Glasser ${ }^{9}$ obtained an expression for the oscillating part of the Knight shift by expanding the free energy, by thermodynamic perturbation theory, in powers of the lattice potential

$$
V(\underline{r})=\Sigma_{K} v_{K} e^{i \underline{k} \cdot \underline{r}}
$$

where $\underline{K}$ is the reciprocal lattice vector. The validity of the calculations is limited only to two regions of magnetic fields: (1) weak fields where $\mu_{0} H \ll v_{K}, \zeta \gg v_{K},(2)$ very high fields where $\mu_{0} H \gg V_{K}, \zeta \gg V_{K}$. An additional condition to be satisfied is $K \neq 2 K_{F}$ where $K_{F}$ is the radius of the underlying free electron sphere. This corresponds to the usual breakdown of the nearly free electron approximation. For moderate field strengths only the steady behavior is obtained. Oscillatory behavior is obtained only at high magnetic fields where the Zeeman energy exceeds the largest energy gap. In this calculation no spin-orbit effects are considered and only the Fermi contact hyperfine interaction is included in the Hamiltonian.

We consider a system of $\mathrm{N}$ non-interacting electrons moving in a weak potential $v(\underline{r})$, which occupies a large volume $\Omega$, in the presence of a nuclear magnetic moment $\mu_{n}$, oriented along an external field represented by the vector potential $\underset{\sim}{A}=\left(-H_{y}, 00\right)$. Each electron is accordingly described by the Hamiltonian

$$
\mathcal{H}=\mathfrak{K}_{L}+\mathcal{K}_{\mathrm{n}}+V(\underline{r})
$$


where

$$
\mathfrak{H}_{L}=\frac{1}{2 m}\left(p-\frac{e}{c} \underline{A}\right)^{2}+2 \mu_{0} H S_{z}
$$

and

$$
\mathcal{H}_{n}=\frac{16 \pi}{3} \mu_{\mathrm{o}} \mu_{n} \delta(\underline{r}) \mathrm{s}_{z}
$$

is the Fermi contact part of the hyperfine interaction.

In $\mathfrak{H}_{2}$ the operator $S_{z}$ commutes with $\mathfrak{K}_{L}$ and the coefficient of $S_{z}$ is a constant. Hence it follows that $S_{z}$ is conserved and also the spin and coordinate variables are separable. $\mathfrak{K}_{L}$ can be written as

$$
\mathcal{H}_{L}=\frac{1}{2 m}\left(p_{x}+\frac{e H}{c} y\right)^{2}+\frac{p_{y}^{2}}{2 m}+\frac{p_{z}^{2}}{2 m}+2 \mu_{0} H s_{z} .
$$

The eigenstates of $\mathcal{H}_{L}$ are the free electron Landau levels ${ }^{48}$

$$
\begin{aligned}
\langle r \mid \lambda\rangle & =\left\langle\underline{r} / k_{x}, n, k_{z}, \sigma\right\rangle \\
& =N_{n} e^{-1 / 2\left(\eta-\eta_{0}\right)^{2}} H_{n}\left(\eta-\eta_{0}\right) e^{i\left(k_{x} x+k_{z} z\right)} x_{\sigma}
\end{aligned}
$$

where $\eta=\left(\frac{e H}{c^{\hbar}}\right)^{1 / 2} y, \eta_{0}=-\left(\frac{e H}{c \hbar}\right)^{1 / 2} k_{x}, N_{n}=\left(\frac{e H}{\pi c^{\hbar}}\right)^{1 / 4} \Omega^{-1 / 3}\left(2^{n+1} n !\right)^{-1 / 2}$, $H_{n}=n^{\text {th }}$ Hermite polynomial and $x_{\sigma}(\sigma= \pm 1)$ are spin states. The corresponding eigen values are

$$
E_{\lambda} \equiv E_{\sigma}\left(n, k_{z}\right)=2 \mu_{0} H\left(n+\frac{1}{2}\right)+\frac{\hbar^{2} k_{z}^{2}}{2 m}+\mu_{0} H \sigma .
$$

To evaluate the free energy we require the expansion of the partition function 


$$
z(\gamma)=\operatorname{Tr}\left\{e^{-\gamma \mathcal{H}}\right\}=\operatorname{Tr}\left\{e^{-\gamma(\mathcal{F}[+\mathcal{H} C+V(\underline{r})}\right\}, \gamma=\frac{1}{k T}
$$

to second order in $V(\underline{r})$ and first order in $K_{n}$ which can conveniently be obtained by using Schwinger trace formula 49

$$
\begin{gathered}
\operatorname{Tr}\left\{e^{Y(A+B)}\right\}=\operatorname{Tr}\left\{e^{-\gamma A}\right\}-\gamma \operatorname{Tr}\left\{B e^{-\gamma A}\right\} \\
+\gamma \sum_{n=2}^{\infty} \frac{(-1)^{n}}{n} \operatorname{Tr}\left\{B e^{-\gamma A} \int_{0}^{Y} d \gamma_{1} \cdot \cdot \cdot \int_{0}^{Y-2} d Y_{n-1} B\left(Y_{1}\right) \cdot \cdot B\left(\gamma_{n-1}\right)\right\}
\end{gathered}
$$

where $B(Y)=e^{-Y A} B e^{Y A}$. Carrying this out to third order, we $f$ ind that the relevant terms are

$$
\begin{aligned}
& z(\gamma)=\left[\operatorname{Tr}\left\{e^{-\gamma \xi \zeta}\right\}+\frac{1}{2} \gamma^{2} \operatorname{Tr}\left\{\int_{0}^{1} d u v(\underline{r}) e^{-\gamma \xi C(1-u)} v(r) e^{-\gamma \xi L u}\right\}\right]
\end{aligned}
$$

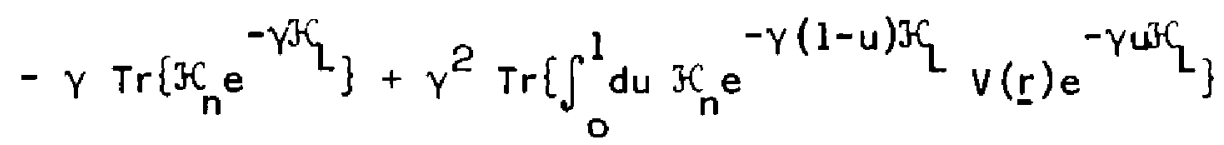

$$
\begin{aligned}
& -\gamma^{3} \operatorname{Tr}\left\{\mathcal{F C}_{n} \int_{0}^{1} d u_{1} \int_{0}^{u_{1}} d u_{2} e^{-\gamma\left(1-u_{1}\right) \mathcal{F C}} v(\underline{r}) e^{-\gamma\left(u_{1}-u_{2}\right) \mathcal{H C}} L_{v(r)} e^{-\gamma u_{2} K L}\right\}
\end{aligned}
$$

+ . . , where the variable of integration has been changed to $Y_{1}=Y u$.

$$
=z_{2}(\gamma)+z_{n}^{(0)}(\gamma)+z_{n}^{(1)}(\gamma)+z_{n}^{(2)}(\gamma)
$$

$z_{2}(\gamma)$ is independent of $\mathcal{F}_{n}$ and gives rise to non-oscillatory magnetic susceptibility. 50 it will not be considered here. We 
first calculate

$$
z_{n}^{(0)}(\gamma)=-\frac{16 \pi}{3} \mu_{0} \mu_{n} \gamma \operatorname{Tr}\left\{\delta(r) s_{z} e^{-\gamma \xi \zeta}\right\} .
$$

If we use Landau states Eq. (4) to evaluate the traces, we obtain for any operator $A$

$$
\begin{aligned}
\operatorname{Tr}\left\{A e^{-\gamma F G}\right\} & =\frac{1}{8 \pi^{2}}\left(\frac{e H}{\pi c h}\right) 1 / 2 \sum_{\sigma} \int_{-\infty}^{\infty} d k \sum_{z=0}^{\infty} \frac{1}{2^{n} n !} \int_{-\infty}^{\infty} d k x \\
& \times M\left(k_{x} n k_{z} \sigma \mid A\right) e^{-\gamma E_{\sigma}}\left(n, k_{z}\right)
\end{aligned}
$$

where

$$
\begin{gathered}
M=\int d^{3} r e^{-1 / 2\left(\eta-\eta_{0}\right)^{2}} H_{n}\left(\eta-\eta_{0}\right) e^{-i\left(k_{x} x+k_{z} z\right)} x_{\sigma} A e^{-1 / 2\left(\eta-\eta_{0}\right)^{2}} \\
H_{n}\left(\eta-\eta_{0}\right) e^{i\left(k_{x} x+k_{z} z\right)} x_{\sigma}
\end{gathered}
$$

where a summation over spin indices is implied in the integration in M. The calculation of free energy is referred to unit volume. Substituting $A=S_{z} \delta(r)$ and noting that the eigen values of $S_{z}$ are $\frac{1}{2} \sigma(\sigma= \pm 1)$ between the spin states $x_{\sigma}$, we get, because of the $\delta$-function

$$
M\left(k_{x} n k_{z} \sigma \mid s_{z} \delta(r)\right)=\frac{1}{2} \sigma e^{-\eta_{0}^{2}} H_{n}{ }^{2}\left(-\eta_{0}\right) .
$$


Putting this into Eq. (8) gives

$$
\begin{aligned}
& \operatorname{Tr}\left\{s_{z} \delta(r) e^{-\gamma 3 G}\right\}=\frac{1}{8 \pi^{2}}\left(\frac{e H}{h_{\pi c}}\right)^{1 / 2} \sum \int_{\sigma}^{\infty} d k x \int_{-\infty}^{\infty} d k z \\
& \times \sum_{n=0}^{\infty} \frac{1}{2^{n} n !} \frac{1}{2} \sigma e^{-\eta_{o_{H}}^{2}}{ }_{H_{n}^{2}\left(-\eta_{0}\right)} e^{-\gamma\left\{2 \mu_{0} H\left(n+\frac{1}{2}\right)+\frac{\hbar^{2} k_{z}{ }^{2}}{2 m}+\mu_{0} H \sigma\right\}} .
\end{aligned}
$$

The $k_{z}$ integration is

$$
\int_{-\infty}^{\infty} d k_{z} e^{-\gamma \frac{\hbar^{2} k_{z}{ }^{2}}{2 m}}=\frac{2 m \pi}{\gamma \hbar^{2}} .
$$

Then

$$
\begin{aligned}
& \operatorname{Tr}\left\{s_{z} \delta(r) e^{-\gamma \xi \xi}\right\}=\frac{1}{8 \pi^{2}}\left(\frac{2 m e H}{\gamma c k^{3}}\right)^{1 / 2} \sum \frac{\sigma}{2} e^{-\gamma \mu_{0} H \sigma} \\
& \times \int_{-\infty}^{\infty} d k_{x} \sum_{n=0}^{\infty} \frac{1}{2^{n} n !} e^{-\eta_{0}^{2}} H_{n}^{2}\left(-\eta_{0}\right) e^{-2 \mu_{0} H_{n}} e^{-\mu_{0} H} .
\end{aligned}
$$

The $\sigma$-sum is $\frac{1}{2}\left(e^{\mu_{0} H y}+e^{-\mu_{0} H y}\right)=\cosh \mu_{0} H y$. To carry the n-summation we use the relation

$$
\sum_{n=0}^{\infty} \frac{1}{2^{n} n !} e^{-1 / 2\left(x^{2}+y^{2}\right)} H_{n}(x) H_{n}(y) e^{-n \alpha}
$$$$
=2^{-1 / 2}(\operatorname{cosech} \alpha)^{1 / 2} e^{\alpha / 2} e^{-\frac{1}{4}\left\{(x+y)^{2} \tanh \left(\frac{\alpha}{2}\right)+(x-y)^{2} \operatorname{coth}\left(\frac{\alpha}{2}\right)\right\}} .
$$ 
Putting

$$
\begin{gathered}
\alpha=2 \gamma \mu_{0} H \\
x=y=\left|\eta_{0}\right|=\left(\frac{c \hbar}{e H}\right)^{1 / 2} k_{x}
\end{gathered}
$$

and using

$$
\int_{-\infty}^{\infty} e^{-\alpha n^{2}} d_{n}=\left(\frac{\pi}{\alpha}\right)^{1 / 2}
$$

we finally have

$$
z_{n}{ }^{(0)}(\gamma)=\frac{8 \pi}{3} \mu_{0} \mu_{n}\left(\frac{m}{2 \pi \hbar^{2}}\right)^{3 / 2} \mu_{0} H^{1 / 2} .
$$

The free energy is given by

$$
F-N S=\Phi=-\frac{1}{2 \pi i} \int_{c-i \infty}^{c+i \infty} s^{-2} Z(s) e^{\zeta s} d s, c>0
$$

and the knight shift by

$$
\sigma=-\frac{1}{H}\left(\frac{\partial F}{\partial \mu_{n}}\right)_{\mu_{n}}=0 .
$$

From Eq. (12) we obtain for the thermodynamic potential

$$
\phi_{n}{ }^{(0)}=-\zeta^{1 / 2}\left(\frac{4}{3 \pi} \mu_{0} \mu_{n}\right)\left(\frac{e H}{c \hbar}\right)\left(\frac{2 m}{\hbar^{2}}\right)^{1 / 2}
$$


and correspondingly for the Knight shift

$$
\sigma_{p}^{(0)}=\frac{8 \pi}{3} x_{p}
$$

where $x_{p}$ is the free electron spin susceptibility. Thus $z_{n}{ }^{(0)}(\gamma)$ gives the Knight shift for free electrons since $\left\langle\left|\psi_{F}(0)\right|^{2}\right\rangle=1$ in this case.

To apply a first order correction to $\sigma_{p}^{(0)}$ we evaluate

$$
\begin{aligned}
& z_{n}^{(1)}(\gamma)=\gamma^{2} \operatorname{Tr}\left[\int_{0}^{1} d u x_{n} e^{-\gamma(1-u) \xi \zeta} v(\underline{r}) e^{-\gamma u F L_{3}}\right. \\
& =\frac{r^{2} e H}{64 \pi^{4} h_{\pi c}} \int_{0}^{1} d u \Sigma \int_{\sigma, \sigma^{\prime}}^{\infty} \int_{-\infty}^{\infty} d k_{x} d k_{x}^{\prime} d k_{z} d k_{z}^{\prime} \sum_{n, n^{\prime}}\left(2^{n} n ! 2^{n^{\prime}} n^{\prime} !\right)^{-1 / 2} \\
& \times M_{1} M_{2} e^{-\gamma u E_{\sigma}\left(n, k_{z}\right)} e^{-\gamma(1-u) E_{\sigma}^{\prime}\left(n^{\prime}, k_{z}^{\prime}\right)}
\end{aligned}
$$

where

$$
\begin{gathered}
M_{1}=\frac{16 \pi}{3} \mu_{0} \mu_{n} \sigma^{\prime} \int d^{3} r e^{-1 / 2\left(\eta-\eta_{0}\right)^{2}} e^{-1 / 2\left(\eta-\eta_{0}^{\prime}\right)^{2}} \\
x e^{i\left[\left(k_{x}^{\prime}-k_{x}\right) x+\left(k_{z}^{\prime}-k_{z}\right) z\right]} H_{n}\left(\eta_{\left.-1-\eta_{0}\right) H_{n}^{\prime}\left(\eta-\eta_{0}^{\prime}\right) \delta(r)}\right. \\
=\frac{16 \pi}{3} \mu_{0} \mu_{n}^{\prime} e^{-1 / 2\left(\eta_{0}^{2}+\eta_{0}^{\prime 2}\right)} H_{n}\left(-\eta_{0}\right) H_{n}^{\prime}\left(-\eta_{0}^{\prime}\right) \\
M_{2}=\sum_{k} v_{k} \int d^{3} r e^{-1 / 2\left(\eta-\eta_{0}\right)^{2}} e^{-1 / 2\left(\eta-\eta_{0}^{\prime}\right)^{2}} e^{i\left[\left(k_{x}-k_{x}^{\prime}\right) x+\left(k_{z}-k_{z}^{\prime}\right) z\right]}
\end{gathered}
$$




$$
\begin{gathered}
\times H_{n}\left(\eta-\eta_{0}\right) H_{n}^{\prime}\left(\eta-\eta_{0}^{\prime}\right) e^{-i \underline{k} \cdot \underline{r}} \\
=(2 \pi)^{2} \sum_{k} v_{k} \delta\left(k_{x}-k_{x}^{\prime}-k_{x}\right) \delta\left(k_{z}-k_{z}^{\prime}-k_{z}\right) \\
x \int_{-\infty}^{\infty} d y e^{-1 / 2\left[\left(\eta-\eta_{0}\right)^{2}+\left(\eta-\eta_{0}^{\prime}\right)^{2}\right.} H_{n}\left(\eta-\eta_{0}\right) H_{n}^{\prime}\left(\eta-\eta_{0}^{\prime}\right) e^{-i k_{y} y} .
\end{gathered}
$$

The $\sigma, \sigma^{\prime}$ sums give $2 \cosh \gamma u \mu_{0} H \sinh \gamma(1-u) \mu_{0} H$. The $n, n^{\prime}$ sums give

$2\left(\sinh 2 \gamma u \mu_{0} H \operatorname{Sinh} 2 \gamma(1-u) \mu_{0} H\right)^{-1 / 2} \exp \left\{-\frac{1}{4}\left[\eta^{2}\left(\operatorname{Coth} \gamma \mu_{0} H+\right.\right.\right.$

$\left.\left.\left.\operatorname{coth} \gamma(1-u) \mu_{0} H\right)+\left(\eta-2 \eta_{0}\right)^{2} \tanh \gamma u \mu_{0} H+\left(I-2 \eta_{0}^{\prime}\right)^{2} \tanh \gamma(1-u) \mu_{0} H\right]\right\}$.

The $k_{z}, k_{z}^{\prime}$ integrals give

$$
\left(\frac{2 \pi m}{\hbar^{2} \gamma}\right)^{1 / 2} e^{-\frac{\hbar^{2} \gamma}{2 m} u(1-u) k_{z}^{2}}
$$

The $k_{x}, k_{x}^{\prime}$ integrals give

$$
\left(\frac{e H \pi}{\pi y}\right)^{1 / 2}(A+B)^{-1 / 2} e^{k_{x}^{2} \frac{B^{2}}{A+B}} e^{-\lambda^{2} k_{x}^{2} B} e^{1 / 4 \eta^{2}(A+B)}
$$

where $A=\tanh \gamma u \mu_{0} H, B=\tanh \gamma(1-u) \mu_{0} H$

$$
\lambda=\left(\frac{\mathrm{c} \hbar}{\mathrm{eH}}\right)^{1 / 2} .
$$


Finally the $y$ integration gives

$$
2\left(\frac{\pi c \hbar}{\mathrm{eH}(C+D)}\right)^{1 / 2} \mathrm{e}^{-\frac{\lambda^{2} K_{y}^{2}}{C+D}}
$$

where $c=\operatorname{Coth} \gamma u \mu_{0} H, D=\operatorname{Coth} \gamma(1-u)_{0} H$. Putting all this together leads to the result, after a great deal of simplification,

$$
\begin{aligned}
& z_{n}^{(1)}(\gamma)=-\gamma^{3 / 2}\left(\frac{e H}{c \hbar}\right)\left(\frac{2 \pi m}{\hbar^{2}}\right)^{1 / 2}\left(\frac{\mu}{6 \pi}\right) \underset{\underline{K}}{\Sigma} v_{K} \int_{0}^{1} d u F(u) \\
& x e^{-\gamma u(1-u) \epsilon_{11}} \stackrel{(K)}{\sim} e^{-\left[\epsilon_{\perp}(\underline{K}) / \mu_{0} H\right] f(u)}
\end{aligned}
$$

where

$$
\epsilon_{11}\left(\underset{\sim}{(K)}=\frac{\hbar^{2} K_{z}^{2}}{2 m}, \epsilon_{\perp} \stackrel{(K)}{\sim}=\frac{\hbar^{2}\left(K_{x}{ }^{2}+K_{y}{ }^{2}\right)}{2 m},\right.
$$

and

$$
\begin{gathered}
F(u)=1+\operatorname{cosech} \gamma \mu_{0} H \operatorname{Sinh} \gamma \mu_{0} H(1-2 u), \\
f(u)=\frac{1}{2} \operatorname{cosech} \gamma \mu_{0} H\left[\cosh \gamma \mu_{0} H-\cosh \gamma \mu_{0} H(1-2 u)\right] .
\end{gathered}
$$

Changing the variable of integration to $S=1-2 u$ we find that the second term coming from $F(u)$ vanishes by symmetry and we are left with

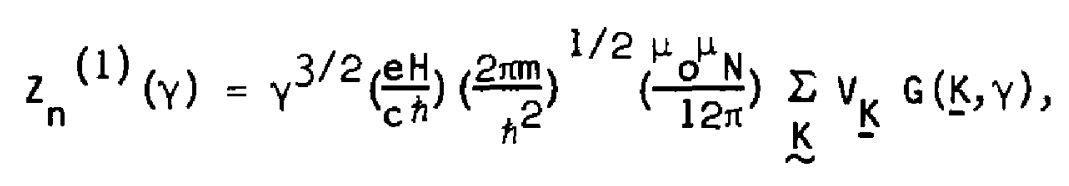


where

$$
\begin{gathered}
G(\underline{K}, \gamma)=\exp \left\{-\frac{\gamma}{4} \epsilon_{11}(\underline{K})-\frac{1}{2}\left[\epsilon_{\perp}(\underline{K}) / \mu_{0} H\right] \operatorname{coth} \gamma \mu_{0} H\right\} \\
\times \int_{0}^{1} d s \exp \left\{\frac{\gamma}{4} \epsilon_{11}(\underline{K}) s^{2}+\left[\frac{\epsilon_{\perp}(\underline{K})}{2 \mu_{0} H}\right] \operatorname{cosech} \gamma \mu_{0} H s\right\} .
\end{gathered}
$$

As can easily be seen from this expression, the first order contribution to the partition function, $z_{n}{ }^{(1)}(\gamma)$, has singularities along the imaginary axis in the complex $\gamma$-plane and, therefore, gives rise to the oscillatory terms in the free energy. For simplicity we assume that $V(\underline{r})$ has only fourier components with wave vectors perpendicular to the magnetic field. Then, introducing the notation

$$
\lambda=\sigma / \mu_{0} H, \quad b=\epsilon(\underline{K}) / \mu_{0} H,
$$

the first order contribution to the free energy may be written as, on replacing $s$ by $s / \mu_{0} H$,

$$
\begin{aligned}
& \phi_{n}^{(1)}=-\frac{1}{2 \pi i} \int_{c-i \infty}^{c+i \infty} s^{-2} z_{n}^{(1)}(s) e^{\zeta s} d s, c>0 \\
& =\frac{1}{4}\left(\mu_{0} H\right)^{-1 / 2}\left[\frac{8 \pi}{3} \mu_{0} \mu_{n}\left(m / 2 \pi \hbar^{2}\right)^{3 / 2} \mu_{0} H\right] \underset{\underline{K}}{\sum} v_{K} I(\underline{K}) ; \\
& 2 \pi i \quad l(\underline{k})=\int_{c-i \infty}^{c+i \infty} d s \int_{0}^{1} d u s^{-1 / 2} \exp [\lambda s+b \operatorname{csch} s(\operatorname{Cosh} s u-\operatorname{Cosh} s)] .
\end{aligned}
$$


By the mean value theorem

$$
\int_{0}^{1} d u \exp [b \operatorname{csch} s \cosh s u]=\exp \left[b \operatorname{csch} s \operatorname{Cosh} s u_{0}\right]
$$

where $0<U_{0}(s)<1$. The singularities at $s=k \pi i$ are therefore essential; however, except at $s=0$, the integrand vanishes if they are approached in the right half-plane, as may be seen by expanding the exponential about one of these points. Consequently, the $s$ contour may be taken to lie along the imaginary axis (the remaining singularity at the origin is integrable). We divide $I(\underline{K})$ into two parts $I=I_{0}+I_{1}$ where $I_{1}$ is the integral from $s=-\pi i$ to $s=\pi i$ and $I_{0}$ is the rest. We further break up $I_{0}$ into a sum of integrals from kri to $(k+1) \pi i,(k \neq-1,0)$, and by means of the substitution $s \rightarrow i(k \pi+s)$ we find that

$$
\begin{aligned}
& 1_{0}=\frac{1}{2 \pi} \sum_{\substack{-\infty \\
k \neq-1,0}}^{\infty} \exp \left(i \lambda \pi+\frac{1}{4} i \pi \operatorname{sgn} k\right) \int_{0}^{\pi} d s \int_{0}^{1} d u|s+k \pi|^{-1 / 2} \\
& \quad x \exp \left\{i \lambda s-i b(-1)^{k} \csc s\left[\operatorname{Cos} u(s+k \pi)-(-1)^{k} \cos s\right]\right\} .
\end{aligned}
$$

The substitution $s \rightarrow i(k \pi+s)$ puts the u-integration in proper form for it to be evaluated by the method of stationary phase which is concerned with integrals of the form

$$
\int_{c} e^{i b f(u)} d u
$$


where $b$ is large and positive and $f(u)$ is real along the contour c. Unless $f^{\prime}(u)=0$, the contributions to the integral from the neighborhood of u will largely cancel because of the rapidly oscillating character of $e^{i b f(u)}$. Thus one looks only for the points along the contour where $f^{\prime}(u)=0$.

The u-integration is

$$
u_{k}(s)=\int_{0}^{1} d u \exp \left[i b h_{k}(s, u)\right],
$$

where $h_{k}(s, u)=(-1)^{k+1} \csc s \cos u(s+k \pi)$, and $b$ is at least of the order of $10^{4}$ or higher. Then, from the method of stationary phase, we have

$$
\begin{gathered}
U_{k}(s)=\Sigma_{j}\left[\frac{2 \pi}{b}\left|h_{k}^{\prime \prime}\left(s, u_{j}\right)\right|\right]^{1 / 2} e_{j} \exp \left[i b h_{k}\left(s, u_{j}\right)+\right. \\
\left.\frac{1}{4} i \pi \operatorname{sgn} h_{k}^{\prime \prime}\left(s, u_{j}\right)\right]+o\left(b^{-1}\right) .
\end{gathered}
$$

The sum is over the roots of $h_{k}^{\prime}(s, u)=0$ which lie in the range of integration and $e_{j}$ is $\frac{1}{2}$ or 1 according as $u_{j}$ is an end point or not. Now, $h_{k}^{\prime}(s, u)=0$ has the roots $u_{j}=j \pi /(s+k \pi)$ for which

$$
\begin{gathered}
h_{k}\left(s, u_{j}\right)=(-1)^{j+k+1} \csc s, \\
h_{k}^{\prime \prime}\left(s, u_{j}\right)=(-1)^{j+k}(s+k \pi)^{2} \csc s,
\end{gathered}
$$

and $i t$ is clear, by inspection, that $j=0,1, \ldots ., k$ for $k>0$ 
and $|j|=0,1, \ldots,|k|-1$ for $k<0$. Also $e_{0}=\frac{1}{2}$ only. In this way we obtain

$$
U_{k}(s) \sim(\operatorname{sgn} k)\left[\frac{2 \pi}{b}(s+k \pi)^{2}\right]^{1 / 2}\left(\frac{1}{2}+k \operatorname{Re}\right) \exp \left[\frac{\pi i}{4}-i b \operatorname{Csc} s\right],(20)
$$

where Re denotes the real part. Inserting Eq. (20) into Eq. (19), we find

$$
\begin{gathered}
\left.2(2 \pi b)^{1 / 2}\right|_{0}=\sum_{-\infty}^{\infty} \exp \left[i \lambda k \pi+\frac{i \pi}{4} \operatorname{sgn} k\right](\operatorname{sgn} k)\left\{e^{i \pi / 4}(k+1)\right. \\
\int_{0}^{\pi} d s(\sin s)^{1 / 2}|s+k \pi|^{-3 / 2} e^{i b g_{1}(s)} \\
\left.+e^{-i \pi / 4} k \int_{0}^{\pi} d s(\sin s)^{1 / 2}|s+k \pi|^{-3 / 2} e^{i b g_{2}(s)}\right\},
\end{gathered}
$$

where $g_{j}(s)=\frac{1}{2}$ as $+\cot s+(-1)^{j} \operatorname{Csc} s$, and $a=46 / \epsilon(\underline{k})$. Now in the second integral replacing $s$ by $(\pi-5)$ and in the corresponding term in the sum over $k$ replacing $k$ by $-(k+1)$ which preserves the condition $k \neq-1,0$; we obtain

$$
\begin{gathered}
\left.(2 \pi b)^{1 / 2}\right|_{0}=\operatorname{Re} \sum_{k \neq-1,0} \exp [i \lambda k \pi+(1+s g n k) \pi i / 4](k+1) \\
(\operatorname{sgn} k) \int_{0}^{\pi} d s(\sin s)^{1 / 2}|s+k \pi|^{-3 / 2} e^{i b g_{1}(s)} .
\end{gathered}
$$

where $a<1$, meaning that the corresponding zone plane does not intersect the Fermi surface, $g_{l}(s)$ has no stationary point in the 
range of integration. The dominant contribution to the integral in Eq. (21) comes, therefore, from the neighborhood of the origin where $g_{1}(s) \sim s$. The integral is thus of order $b^{-3 / 2}$, which is completely negligible since $b$ is very large. When $a>1$, on the other hand, $g_{l}(s)$ is stationary at $s=s_{0}$ where

$$
\begin{aligned}
& \operatorname{Cos} s_{0}=(2-a) / a \\
& g_{1}\left(s_{0}\right)=\frac{1}{2} a s_{0}-(a-1)^{1 / 2} \\
& g_{1}^{\prime \prime}\left(s_{0}\right)=-\frac{1}{2} a(a-1)^{1 / 2} .
\end{aligned}
$$

We find, again by the method of stationary phase,

$$
\begin{gathered}
\int_{0}^{\pi} d s(\sin s)^{1 / 2}|s+k \pi|^{-3 / 2} e^{i b g_{1}(s)} \sim \\
{\left[\frac{2 \pi}{b}\right]^{1 / 2}\left(\frac{2}{a}\right)\left|s_{o}+k \pi\right|^{-3 / 2} \exp \left[i g_{1}\left(s_{o}\right)-\frac{1}{4} \pi i\right],}
\end{gathered}
$$

which gives

$$
\begin{aligned}
I_{0}= & \frac{2}{a b} \sum_{k=1}^{\infty}\left\{\frac{(k+1)}{\left|s_{0}+k \pi\right|^{3 / 2}} \cos \left[\lambda k \pi+b g_{1}\left(s_{0}\right)+\frac{1}{4} \pi\right]\right. \\
& \left.+\frac{(k-1)}{\left|k \pi-s_{0}\right|^{3 / 2}} \cos \left[\lambda k \pi-b g_{1}\left(s_{0}\right)+\frac{1}{4} \pi\right]\right\}
\end{aligned}
$$

where the symmetry of the two terms in the interchange of $s_{0}$ and -s $_{0}$ is to be observed. 
We next require $1_{1}$ which may be written

$$
\pi I_{1}=I_{m} \int_{c^{+}} d s \int_{0}^{1} d u s^{-1 / 2} \exp [\lambda s+b \operatorname{Csch} s(\operatorname{Cosh} s u-\operatorname{Cosh} s)],
$$

where $I_{m}$ denotes the imaginary part and $c^{+}$is a path, lying in the right hand $s$ plane, from anywhere on the real axis to $\pi i$. We now let

$$
U=\int_{0}^{1} \exp [\operatorname{xh}(s, u)] d u
$$

where $|x|=|b \operatorname{csch} s| \gg 1$ on $c^{+}$and $h(s, u)=$ Cosh su. Considering $U$ by the method of steepest descents, we find that $U=0$ is a saddle point of order unity. Writing $s=\sigma e^{i \theta}$ and $u=\alpha+i \beta$, it is not difficult to show that the steepest paths through this point, given by $I_{m} h(s, u)=0$, are

$$
\begin{aligned}
& \beta=\alpha \tan (\pi / 2-\theta), \\
& \beta=-\alpha \tan \theta,
\end{aligned}
$$

out of which one can choose any one which is suitable for the purpose of integration. If we distort the $u$ contour to run along the former, we find that

$$
U \sim \frac{1}{2} i[2 \pi /|x|]^{1 / 2}\left(e^{x / s}\right),
$$

and Eq. (24) becomes

$$
\left.(2 n b)^{1 / 2}\right|_{1} \approx \operatorname{Re} \int_{c^{+}} d s s^{-3 / 2}|\sinh s|^{1 / 2} e^{b g(s)},
$$


92

where $g(s)=\frac{1}{2}$ as $-\operatorname{Coth} s+\operatorname{csch} s$. For $a<1, g^{\prime}(s)=0$ has $a$ real positive root $\sigma_{0}$ given by $\cosh \sigma_{0}=(2-a) / a$ which may be chosen as the endpoint of $\mathrm{c}^{+}$, so this term exhibits only steady behavior. For a $>1, \sigma_{0}=$ is, where $s_{0}$ is described in Eq. (22), and in addition $g(i x)=i g_{1}(x)$. The steepest paths have zero slope on the imaginary s axis; so distorting the contour to pass horizontally through $s=i s_{0}$, we find

$$
\begin{gathered}
\int_{c^{+}} d s|\sinh s|^{1 / 2} s^{-3 / 2} e^{b g(s)} \\
\sim \frac{2}{a} s_{0}^{-3 / 2}\left[\frac{2 \pi}{b}\right]^{1 / 2} \exp \left[i g_{1}\left(s_{0}\right)-3 i \pi / 4\right],
\end{gathered}
$$

and

$$
I_{1} \simeq\left[2 / a b s_{0}^{3 / 2}\right] \cos \left[b g_{1}\left(s_{0}\right)+\frac{1}{4} \pi\right]
$$

Combining Eqs. (18), (23) and (25) we find that, for $a(k)>1$, the oscillatory part of $\sigma_{n}(1)$ is given by

$$
\sigma_{n}^{(1)}=\frac{1}{2} \sqrt{\mu} H\left[\frac{8 \pi}{3} \mu_{0}^{2}\left(m / 2 \pi \hbar^{2}\right)^{3 / 2}\right] \sum_{k}^{1}\left(v_{k} / \zeta\right)\left[s_{0}^{-3 / 2} \cos \left[\pi \xi_{0} / \mu_{0} H+\pi / 4\right]\right.
$$

$$
-\sum_{k=1}^{\infty}\left\{\frac{(k+1)}{\left|k \pi+s_{0}\right|^{3 / 2}} \cos \left[\pi \xi_{k}^{+} / \mu_{0} H^{+} \pi / 4\right]+\right.
$$

$$
\left.\left.\frac{(k-1)}{\left|k \pi-s_{0}\right|^{3 / 2}} \cos \left[\pi \xi_{k}^{-} / \mu_{0} H+\pi / 4\right]\right\}\right],
$$

where $\xi_{0}=\frac{1}{2 \pi} \epsilon \underset{\sim}{(K) g_{l}}\left(s_{0}\right)$ and $\xi_{k}^{ \pm}=k \delta \pm \xi_{0}$. 
To discuss the oscillatory effects, we require an interpretation of Eq. (26). This is easily obtained with reference to Fig. (20). We find from Eq. (22),

$$
\cos ^{2} \frac{1}{2} s_{0}=\frac{1+\cos s_{0}}{2}=\frac{1}{a}=\frac{(\underline{k})}{4 \zeta}=\frac{k^{2}}{4 k_{F}^{2}},
$$

$\cos \frac{1}{2} s_{0}=K / 2 k_{F}$, and $s_{0}$ may be identified as the angular aperture of the overlap, or lens, surface as seen from the center of the zone. The area of the lens is

$$
\begin{aligned}
A_{L} & =k_{F}^{2}\left(s_{0}-\sin s_{0}\right)=\frac{k^{2} a}{4}\left(s_{0}-\frac{2}{a}(a-1)^{1 / 2}\right) \\
& =\frac{1}{2} k^{2}\left[\frac{1}{2} a s_{0}-(a-1)^{1 / 2}\right]=\frac{1}{2} k^{2} g_{1}\left(s_{0}\right) .
\end{aligned}
$$

Consequently, the frequency $f_{L}$ of the oscillation corresponding to the effective Fermi level $\xi_{o}$, is given by $\left(\hbar_{c} / 2 \pi e\right) A_{L}$, and the oscillation may be identified as due to the lens orbit. The oscillation in $\xi_{k}^{+}$is due to an electron making k circuits of the Fermi sphere before tunneling to the lens, and the oscillation in $\xi_{k}^{-}$corresponds to an electron circling the Fermi sphere $(k-1)$ times and then following the dumbbell orbit which is a possible orbit according to Fig. (20). The giant orbit oscillation also occurs, but its amplitude is smaller than the others by a factor of $10^{4}$. To obtain oscillations corresponding to more complicated orbits, as well as the de Haas van-Alphen oscillation when $a(\underline{k})<1$, requires going to higher order in $V(\underline{r})$. 


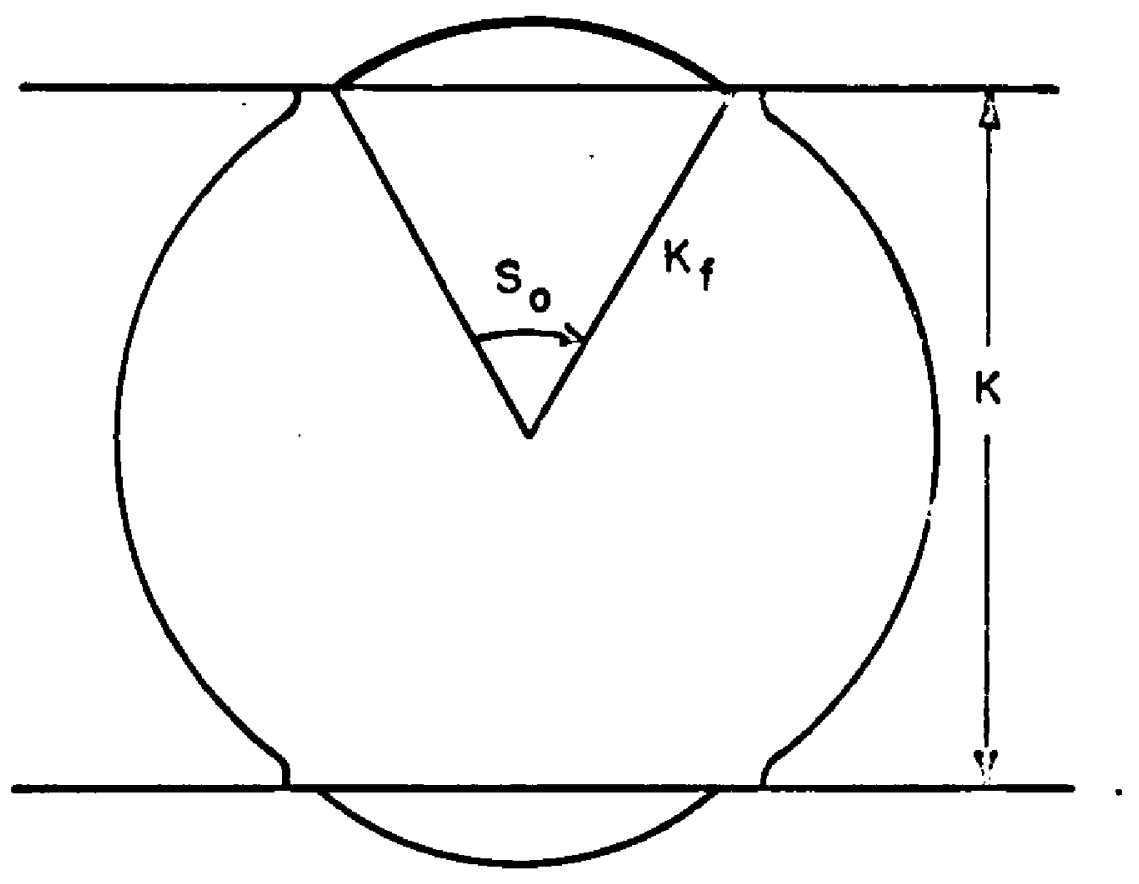

Fig. 20 
It is clear that in first order, the oscillatory behavior is dominated by the lens orbit oscillation $\sigma_{L}$ for which we have

$$
\begin{gathered}
r=\frac{a m p \sigma_{L}}{\sigma(H=0)}=\frac{a m p \sigma_{L}}{\frac{8 \pi}{3} x_{p}} \\
=\frac{\frac{1}{2}\left(\mu_{o} H\right)^{1 / 2}\left[\frac{8 \pi}{3} \mu_{o}^{2}\left(m / 2 \pi \hbar^{2}\right)^{3 / 2}\right] \frac{\delta}{\zeta} s_{o}^{-3 / 2}}{\left(\frac{4 \mu_{o}}{3}\right)\left(\frac{e}{\pi \hbar}\right)\left(\frac{2 m \zeta}{\hbar^{2}}\right)^{1 / 2}} \\
r=\frac{\delta\left(\pi \mu_{0} H\right)^{1 / 2}}{8\left(\zeta s_{o}\right)^{3 / 2}}=\frac{\delta\left(\mu_{0} H\right)^{1 / 2}}{8 \pi(\zeta \theta)^{3 / 2}}
\end{gathered}
$$

where $\delta$ is the band gap corresponding to the zone boundary across which overlap occurs, and $\pi \theta$ is the angular extent of the overlapped portion of the electron sphere, as measured from its center. Taking typical values of $\delta \simeq 0.1 \mathrm{eV}, \mu_{\mathrm{o}} \mathrm{H}=10^{-4} \mathrm{eV}, \delta \simeq 10 \mathrm{eV}$, we find $r=s_{0}^{-3 / 2} \times 10^{-5}$. The effect will clearly be dominated by the oscillation for the zone plane which has the smallest overlap, and thus the smallest value of $s_{0^{*}}$ An overlap surface subtending an angle of a few degrees, which is not unreasonable to expect in tin, would give an $r$ of the observed magnitude. It is to be noticed that the amplitude of the oscillations grows as $H^{1 / 2}$ with the increase of the magnetic field $\mathrm{H}$.

E. Theory of Dolgopolov and Bystrik:

The Hamiltonian of the interaction between the nucleus and the s-type conduction electrons in an external magnetic field 
$H$ in the $z$-direction can be written as:

$$
\mathcal{H}=g \mu_{0} I_{z} H-\frac{8 \pi}{3} g \mu_{0} ! \cdot \sum_{k} \underline{\mu}_{k} \delta\left(\underline{r}_{k}\right)
$$

where $\mu_{n}=g \mu_{0} l$ is the magnetic moment of the nucleus, and $\mu_{k}$ is the magnetic moment of the $k^{\text {th }}$ conduction electron; $\mu_{0}=$ nuclear magneton, $I=$ nuclear spin, $g=g$-factor. The summation is over all the conduction electrons. Considering that the magnetic moment of the electrons is negative, the expectation value of the Hamiltonian can be written as

$$
E_{m}=-g \mu_{0} m_{1} H+\frac{16 \pi}{3} \beta g \mu_{0} m_{1} \sum_{k}\left|\Psi_{k}(0)\right|^{2} m_{s k}
$$

where $m_{1}=\left\langle 1_{z}\right\rangle, m_{s}=\left\langle s_{z}\right\rangle,\left|\psi_{k}(0)\right|^{2}$ is the probability density of the $k^{\text {th }}$ conduction electron at the site of the nucleus, and $\beta$ is the Bohr magneton. One may write

$$
E_{m}=-g \mu_{0} m_{1} H(1+\sigma)
$$

where $\sigma$, the Knight shift is

$$
\sigma=\frac{16 \pi \beta}{3 H} \sum_{k}\left|\psi_{k}(0)\right|^{2}\left(-m_{s k}\right)
$$

For free electrons in a uniform magnetic field one can write for the wave function

$$
\psi(x, y, z)=x(y) \exp \left[\frac{i}{\hbar}\left(x p_{x}+z p_{z}\right)\right]
$$




$$
\begin{gathered}
=\frac{1}{L_{1} L_{3}} \varphi_{n, p_{z}}\left(y+\frac{C p_{x}}{e H}\right) \exp \left[i\left(x p_{x}+z p_{z}\right) / \hbar\right] \\
\int_{-\infty}^{\infty}|\varphi(y)|^{2} d y=1
\end{gathered}
$$

where $L_{1}$ and $L_{3}$ are the dimensions of the sample in the direction of $x$ and $z$ axes. The number of states in the interval $\Delta p_{x} \Delta p_{z}$ for a given value of the quantum number $n$ and a given direction of the spin is, in the semi-classical approximation, equal to

$$
(2 \pi \hbar)^{-2} L_{1} L_{3} \Delta p_{x} \Delta p_{z}
$$

By transforming in Eq. (3) from a summation over conduction electrons to a summation over all possible states of an individual electron and letting $L_{1}$ and $L_{3}$ go to infinity, one may replace the sum over the possible values of $p_{x}$ and $p_{z}$ by an integral. Then

$$
\sigma=\frac{4 \beta e}{3 \pi c \hbar^{2}} \sum_{\text {spin }}\left(-m_{s}\right) \sum_{n=0}^{\infty} \int_{-\infty}^{\infty} d p_{z} f\left(\frac{E_{n}\left(p_{z}\right)-\epsilon}{k T}\right)
$$

where $f(x)=\left(e^{x}+1\right)^{-1}$ is the Fermi distribution function, $\epsilon=\zeta \mp \beta H, \zeta=$ chemical potential, $k=$ Boltzmann constant and $T$ = absolute temperature. Equation (4) may be written as

$$
\sigma=\frac{4 \beta e}{3 \pi c \hbar^{2}} \sum_{\text {spin }}\left(-m_{s}\right) \sum_{n=0}^{\infty} \varphi(n) .
$$


Using Poisson's summation formula, ${ }^{51}$ this could be written as

$$
\begin{gathered}
\sigma=\frac{4 \beta e}{3 \pi c \hbar^{2}} \sum_{\text {spin }}\left(-m_{s}\right)\left\{\frac{1}{2} \varphi(0)+\int_{0}^{\infty} \varphi(n) d n\right. \\
\left.+2 \sum_{k=1}^{\infty} \int_{0}^{\infty} \varphi(n) \cos 2 \pi k n d n\right\} .
\end{gathered}
$$

The dependence of the energy of a quasi-particle in the magnetic field on the quantum number $n$ has, in the semi-classical approximation, the form

$$
S\left(E, P_{z}\right)=(n+\gamma) 2 \pi e H \hbar / c, \quad(0<\gamma<1)
$$

where, for the case of a quadratic dispersion law, $\gamma=\frac{1}{2}$. Thus, in as much as $\gamma \rightarrow \frac{1}{2}$ for the case of a free electron, Eq. (5) can be written as

$$
\sigma=\frac{4 \beta e}{3 \pi c \hbar^{2}} \sum_{\operatorname{spin}}\left(-m_{s}\right)\left\{\int_{-\frac{1}{2}}^{\infty} \varphi(n) d n+2 \operatorname{Re} \sum_{k=1}^{\infty} \int_{-\frac{1}{2}}^{\infty} d n \varphi(n) e^{2 \pi k i n}\right\}
$$

where Re denotes the real part. Writing $k T=\theta$, for the sake of clarity, we have

$$
\begin{gathered}
\sigma=\frac{4 \beta e}{3 \pi c \hbar^{2}} \sum_{\text {spin }}\left(-m_{s}\right)\left\{\int_{-\frac{1}{2}}^{\infty} d n \int_{-\infty}^{\infty} d p_{z} f\left(\frac{E_{n}-\epsilon}{\theta}\right)\right. \\
\left.+2 \operatorname{Re} \sum_{k=1}^{\infty} \int_{-\frac{1}{2}}^{\infty} d n \int_{-\infty}^{\infty} d p_{z} f\left(\frac{E_{n}-\epsilon}{\theta}\right) e^{2 \pi i n k}\right\} .
\end{gathered}
$$


Since, from Eq. (6)

$$
\frac{\partial n}{\partial E}=\frac{c}{2 \pi e^{h}} \frac{\partial S}{\partial E}
$$

we have

$$
\begin{array}{r}
\sigma=\frac{4 \beta e}{3 \pi c \hbar^{2}} \frac{c}{2 \pi e H^{\hbar}} \sum_{s p i n}\left(-m_{s}\right)\left(\int_{0}^{\infty} d E f\left(\frac{E-E}{\theta}\right) \frac{d}{d E} \int_{s>0} S\left(E, p_{z}\right) d p_{z}\right. \\
\left.+2 \operatorname{Re} \sum_{k=1}^{\infty} \int_{0}^{\infty} d E f\left(\frac{E-\epsilon}{\theta}\right) \int_{s>0} d p_{z} \frac{\partial S}{\partial E} \exp \left[\frac{i k c}{e H^{\hbar}} S\left(E, p_{z}\right)-2 \pi i k \gamma\right]\right\} .
\end{array}
$$

The first term in the curly brackets is

$$
J_{1}=\int_{0}^{\infty} d E f\left(\frac{E-\epsilon}{\theta}\right) \frac{d U}{d E},
$$

where

$$
U=\int_{E>0} S\left(E, P_{z}\right) d p_{z}
$$

is the volume bounded by the surface of constant energy $E$ in momentum space. Writing $J_{1}=J_{1}(\epsilon)=J_{1}(\zeta \mp B H)$, we have

$$
J_{1}=J_{1}(\zeta) \mp \beta H \frac{\partial J_{1}(\zeta)}{\partial \zeta}+\ldots \cdot
$$

since $B H \ll 6$. Now for $\theta \ll 6$, one can write

$$
J_{1}(6)=\int_{0}^{6} d E \frac{\partial U}{\partial E}
$$


which implies

$$
\frac{\partial J_{1}(\zeta)}{\partial \zeta}=\frac{\partial U(\zeta)}{\partial \zeta}
$$

The refore,

$$
\mathrm{J}_{1}=\mathrm{J}_{1}(\zeta) \mp B \mathrm{BH} \frac{\partial \mathrm{U}(\zeta)}{\partial \zeta}
$$

Hence, for the Knight shift caused by the first term in Eq. (8), we have

$$
\begin{aligned}
\sigma_{p} & =\frac{8 \pi}{3} \beta^{2} \frac{2}{(2 \pi \hbar)^{3}} \frac{\partial U(\zeta)}{\partial \zeta} \\
& =\frac{8 \pi}{3} x_{p}
\end{aligned}
$$

where $X_{p}$ is the paramagnetic susceptibility. The second term in Eq. (8) describes the diamagnetic part $\sigma_{d}$ of the Knight shift. This is

$$
\begin{gathered}
\sigma_{d}=\frac{4 \beta}{3 \pi^{2} \hbar^{3} H} \sum_{s p i n}\left(-m_{s}\right) \operatorname{Re} \sum_{k=1}^{\infty} \int_{0}^{\infty} d E f\left(\frac{E-\epsilon}{\theta}\right) \\
\times \int_{s>0} d p_{z} \frac{\partial S}{\partial E} \exp \left[\frac{i k c}{e H \hbar} s\left(E, p_{z}\right)-2 \pi i k \gamma\right] .
\end{gathered}
$$

This integral occurs in the oscillatory part of the magnetic susceptibility and has been evaluated by Lifshitz and Kosevich. 22 
We write the result

$$
\begin{gathered}
\sigma_{d}=\frac{8 \beta}{3 \pi \hbar^{3}}(2 \pi)^{-1 / 2}\left(\frac{e \hbar}{c}\right)^{3 / 2} H^{1 / 2} \sum_{s p i n}\left(-m_{s}\right) \sum_{k=1}^{\infty} \frac{\psi(k \lambda)}{k^{3 / 2}}\left|\frac{\partial^{2} s\left(\epsilon, p_{z}\right)}{\partial p_{z}^{2}}\right|_{m}^{-1 / 2} \\
x \sin \left(\frac{k c}{e H^{\hbar}} s_{m}(\epsilon)-2 \pi k \gamma \mp \frac{\pi}{4}\right)
\end{gathered}
$$

where

$$
\psi(k \lambda)=\frac{k \lambda}{\sin k \lambda}, \lambda=\frac{\pi c k T}{e^{h}} \frac{\partial S_{m}(\epsilon)}{\partial \epsilon},
$$

and $S_{m}$ is the area of the extremal cross-section. To accomplish the sum over spin we expand $s_{m}(\epsilon)$ in the argument of the sine function in powers of $\beta H$, limiting the expansion to terms of the first order,

$$
S_{m}(\epsilon)=S_{m}(\zeta) \mp \beta H \frac{\partial S_{m}(\zeta)}{\partial \zeta} ;
$$

in the remaining coefficients we can replace $\epsilon$ with $\sigma_{0}$, the chemical potential in the absence of the magnetic field. The final expression for the oscillatory Knight shift is

$$
\begin{gathered}
\sigma_{\text {osc }}=\frac{8 \beta}{3 \pi}(2 \pi)^{-1 / 2}\left(\frac{e}{c^{\hbar}}\right)^{3 / 2} H^{1 / 2}\left|\frac{\partial^{2} s}{\partial p_{z}^{2}}\right|_{m}^{-1 / 2} \sum_{k=1}^{\infty} \frac{\psi(k \lambda)}{k^{3 / 2}} \cos \left[\frac{k}{2 m_{0}} \frac{\partial S\left(\zeta_{0}\right)}{\partial \zeta_{0}}\right] \\
\times \sin \left[\frac{k c}{e^{\hbar}} s_{m}\left(\sigma_{o}\right) \mp \frac{\pi}{4}-2 \pi k \gamma\right]
\end{gathered}
$$


From this it follows that the amplitude of the oscillations in the Knight shift grows as $H^{1 / 2}$. In order to estimate the order of magnitude of the amplitude of the Knight shift oscillations, we compare Eq. (11) with the expression for the oscillatory magnetization 22

$$
\begin{aligned}
M_{\text {osc }} & =-4(2 \pi)^{-5 / 2}\left(\frac{e}{c \hbar}\right)^{3 / 2} S_{m}(\zeta) H^{1 / 2}\left|\frac{\partial^{2} s\left(\zeta, p_{z}\right)}{\partial p_{z}^{2}}\right|_{m}^{-1 / 2}\left|\frac{\partial S_{m}(\zeta)}{\partial \zeta}\right|^{-1} \\
& \times \sum_{k=1}^{\infty} \frac{\psi(k \lambda)}{k 3 / 2} \sin \left[\frac{k c}{e^{\hbar}{ }^{\hbar}} S_{m}(\zeta) \mp \frac{\pi}{4}-2 \pi k \gamma\right] \cos \left[\frac{k}{2 m_{0}} \frac{\partial S_{m}(\zeta)}{\partial \zeta}\right]
\end{aligned}
$$

and obtain

$$
\sigma_{\text {osc }}=\frac{8 \pi}{3} \beta \frac{\partial S_{m}(\zeta)}{\partial \zeta} \frac{M_{\text {osc }}}{S_{m}(\zeta)} .
$$

Since the oscillations of magnetic susceptibility are of the order of the magnetic susceptibility itself, one may write $M_{\text {osc }}=x_{p} H$. Then

$$
\begin{aligned}
\sigma_{o s c} & \simeq \beta H \frac{\partial S_{m}(\zeta)}{\partial \zeta} \frac{1}{S_{m}(\zeta)} \sigma_{p} \\
& \approx \frac{\beta H}{\zeta} \sigma_{p} .
\end{aligned}
$$

For $\beta=10^{-20} \mathrm{erg} /$ gauss, $H \simeq 10^{4}$ gauss, and for $\operatorname{tin} \zeta \approx 1.58 x$ $10^{-11}$ erg, $\sigma_{p} \approx 7.5 \times 10^{-3}$, we have from Eq. (13)

$$
\sigma_{\text {osc }} \approx 10^{-7}
$$


which is smaller by a factor of about 400 compared to the observed amplitude. 


\section{REFERENCES}

1. J. M. Reynolds, R. G. Goodrich, and S. A. Khan, Phys. Rev. Letters 16,609 (1966).

2. C. H. Townes, C. Herring, and W. D. Knight, Phys. Rev. I7, $852(1950)$.

3. T. P. Das and E. H. Sondheimer, Phil. Mag. 2, 529 (1960).

4. J. I. Kaplan, J. Phys. Chem. Solids 23, 826 (1962).

5. S. Rodriguez, Phys. Letters 4, 306 (1963).

6. J. M. Stephen, Phys. Rev. 123, 126 (1961).

7. D. G. Dolgopolov and P. S. Bystrik, Zh. Eksperim. i Teor. Fiz. 46, 593 (1964). Translation: Soviet Physics JETP 19, $404(1964)$.

8. M. H. Cohen and L. M. Falicov, Phys. Rev. Letters 2, 544 (1960).

9. M. L. Glasser, Phys. Rev. 150, 234 (1966).

10. B. R. MacGarvey and H. S. Gutowsky, J. Chem. Physics 21, $2114(1953)$

11. N. Bloembergen and T. J. Rowland, Acta Met. 1,731 (1953).

12. Yu. S. Karimov and 1. F. Shchegolev, J. Exptl. Theoret. Phys. (U.S.S.R.), 40, 1289 (1961). Translation: Soviet Phys. JETP 13, 899 (1961).

13. E. P. Jones and D. L1. Williams, Phys. Letters 1 , 109 (1962).

14. E. P. Jones and D. L1. Williams, Canadian J. Phys. 42, 1499 (1964). 
15. G. D. Watkins, Ph.D. Thesis (Unpublished), Harvard University, Cambridge, Massachusetts, (1952); E. P. Jones, Ph. D. Thesis, University of British Columbia, Vancouver 8, Canada (1963).

16. D. Shoenberg and P. J. Stiles, Pro. Roy. Soc. (London) A281, 62 (1964).

17. L. R. Windmiller, Phys. Rev. 149, 472 (1966).

18. A. V. Gold and M. G. Priestley, Phil. Mag. 2, 1089 (1960).

19. G. Weisz, Phys. Rev. 149, 504 (1966).

20. M. L. Glasser, J. Math. Phys. 2, 1150 (1964).

21. M. L. Glasser, J. Math. Phys. 43, 158 (1964).

22. 1. M. Lifshitz and A. M. Kosevich, Zh. Eksperim. i Teor. Fiz. 29, 730 (1955). Translation: Soviet Physics JETP 2, 636 (1956).

23. S. Mase, J. Phys. Soc. Japan 14, 1538 (1959).

24. M. Miasek, Phys. Rev. 130, 11 (1963).

25. A. G. Redfield, Phys. Rev. 101, 67 (1956).

26. P. S. Allen and E. F. W. Seymour, Proc. Phys. Soc. $\underline{82}, 174$ (1963).

27. Y. Yafet, J. Phys. Chem. Solids 2l, 99 (1961).

28. J. E. Hebborn, Proc. Phys. Soc. 80,1237 (1962).

29. Cominco Products Inc., Spokane, Washington; F. L. Moseley Co., Pasadena, California; Hewlett-Packard Co., Palo Al to, California; Magnion Inc., Burlington, Massachusetts; McIntosh Laboratory Inc., Binghamton, N.Y.; Tektronix, Inc., Beaverton, Oregon.

30. R. Blume, Rev. Sci. Instr. 32, 743 (1961).

31. G. B. Robinson and F. E. Geiger, Jr., Rev. Sci. Instr. $\underline{2}$, 730 (1958). 
32. J. M. Mays, H. R. Moore, and R. G. Shulman, Rev. Sci. Instr. 29, 300 (1958).

33. E. P. Vol'skii, Soviet Physics JETP 16, 792 (1963).

34. E. P. Vol'skii, Soviet Phys. JETP 19, 89 (1964).

35. V. F. Gantmakher, Zh. Eksperim. i Teor. Fiz. 444, 811 (1963). Translation: Soviet Phys. JETP 17, 549 (1963).

36. V. F. Gantmakher, Zh. Eksperim. i Teor. Fiz. 46, 2028 (1964). Translation: Soviet Phys. JETP 19, 1366 (1964).

37. N. E. Alekseevski, Yu. P. Gaidukov, 1. M. Lifshitz, and V. G. Peschanskii, Zh. Eksper. i Teor. Fiz. 39, 1201 (1960). Translation: Soviet Phys. JETP 12, 837 (1961).

38. N. E. Alekseevski $i$ and Yu P. Gaidukov, Zh. Eksper. i Teor. Fiz. 41, 1079 (1961). Translation: Soviet Phys. JETP $\underline{14}$, 770 (1962).

39. R. C. Young, Phys. Rev. Letters 15, 262 (1965).

40. A. A. Galkin, E. A. Kaner, and A. P. Korolyuk, Zh. Eksperim. i Teor. Fiz. 39, 1517 (1960). Translation: Soviet Phys. JETP $\underline{12}, 1055$ (1961).

41. T. Olson, J. Phys. Chem. Solids 24, 649 (1963).

42. R. J. Kearney, A. R. Mackintosh, and R. C. Young, Phys. Rev. 140, Al671 (1965).

43. M. S. Khaikin, Zh. Eksperim. i Teor. Fiz. 르, 27 (1962). Translation: Soviet Phys. JETP 15, 18 (1962). Zh. Eksperim. i Teor. Fiz. 39, 513 (1960). Translation: Soviet Phys. JETP 12, 359 (1961). 
44. J. F. Koch and A. F. Kip, Phys. Rev. Letters $\underline{8}, 473$ (1962).

45. K. C. Bordoloi, Ph. D. Thesis (Unpublished), Louisiana State University, Baton Rouge, Louisiana (1964).

46. See for example, F. J. Milford, Amer. J. Phys. 28, 521 (1960).

47. E. H. Sondheimer and A. H. Wilson, Proc. Roy. Soc. (London) A210, 173 (1951).

48. L. D. Landau and E. M. Lifshitz, Quantum Mechanics (AddisonWes ley Publishing Co., Reading, Massachusetts, 1958), Sect. 125.

49. A. Saenz and R. O'Rourke, Rev. Mod. Phys. 27, 381 (1955).

50. M. L. Glasser, Phys. Rev. 134, Al296 (1964).

51. R. Courant and D. Hilbert, Methods of Mathematical Physics (Interscience Publishing Co., New York, 1953), Vol. I. 
VITA

Sharif Ahmad Khan was born July 1, 1933 in V. Sathla, District Meerut, India. He graduated from Jat Vedic H.S. Mawana (Meerut) in 1951, received his B.S. from Agra University (India) in 1955 and his M.S. in Physics from Aligarh Muslim University, Aligarh (India) in 1957. He was on the faculty of the Physics Department of Aligarh Muslim University as a lecturer from July 1957 to July 1963 and was married to the former Farhat Jahan in January of 1963. He came to U.S.A. as a Fulbright Fellow in August 1963 and entered the Graduate School of Louisiana State University, Baton Rouge, Louisiana, in September of the same year. He has been elected to the Scholastic Honorary Society of Phi Kappa Phi and is now a candidate for the degree of Doctor of Phi losophy in the Department of Physics and Astronomy of Louisiana State University. 
Candidate: Sharif Ahmad Khan

Major Field: Physics

Title of Thesis: Oscillatory Field Dependence of the Knight Shift in a Monocrystal of Tin

\section{Approved:}

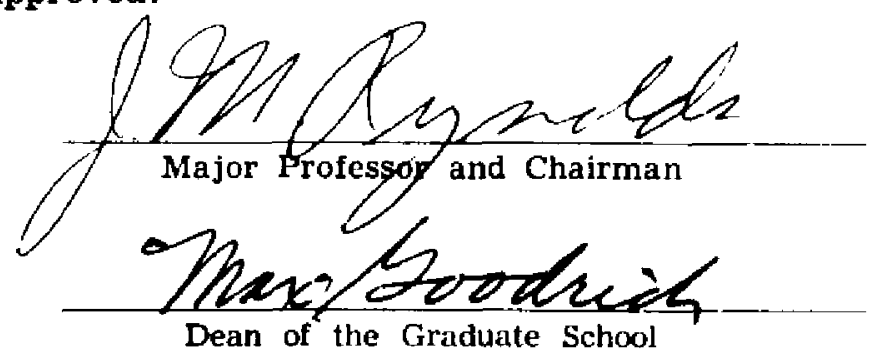

EXAMINING COMMITTEE:

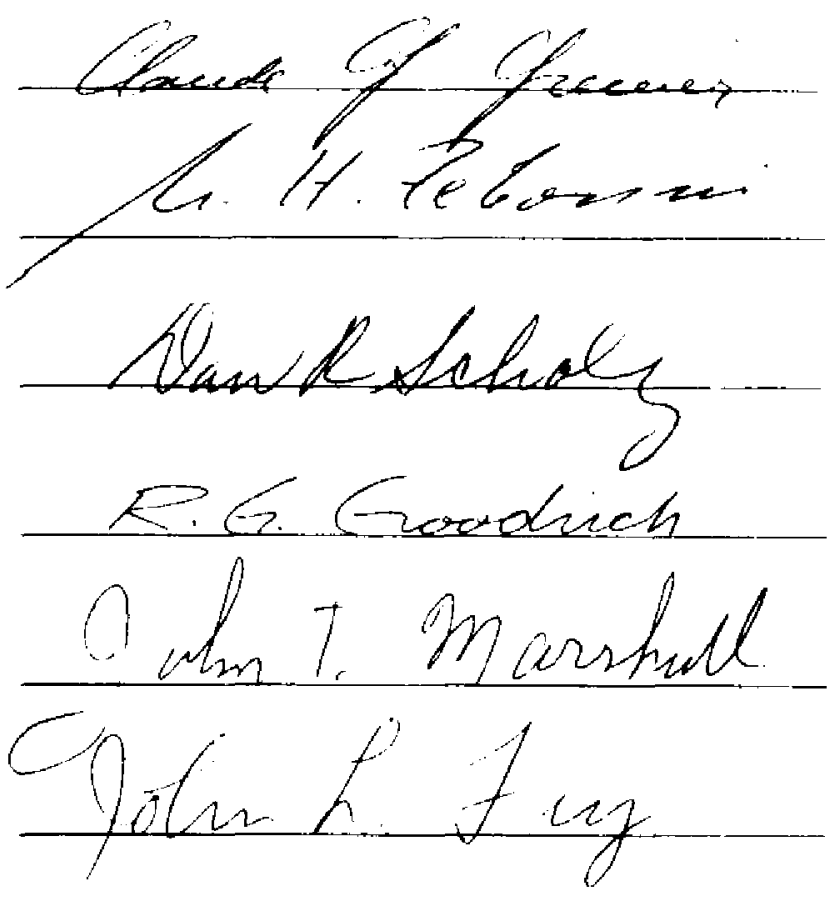

Date of Examination:

January 23,1967 